\title{
JUN 51972
}

Technical Note $\mathrm{N}-1213$

CORROSION OF MATERIALS IN SURFACE SEAWATER

AFTER 12 AND 18 MONTHS OF EXPOSURE

By

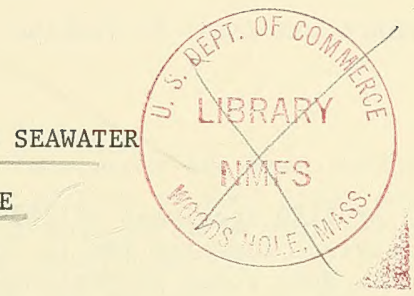

Fred M. Reinhart and James F. Jenkins

January 1972
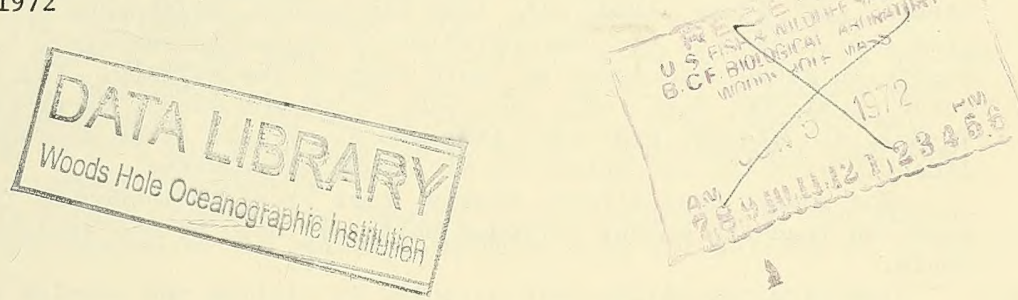

Approved for public release;

distribution unlimited

NAVAL CIVIL ENGINEERING LABORATORY

Port Hueneme, California 93043

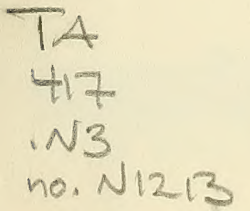


CORROSION OF MATERIALS IN SURFACE SEAWATER AFTER 12 AND 18 MONTHS OF EXPOSURE

Technical Note $\mathrm{N}-1213$

YF 38.535 .005 .01 .004

by

Fred M. Reinhart and James F. Jenkins

\section{ABSTRACT}

A total of 1150 specimens of 189 different alloys were completely immersed in surface seawater for 12 and 18 months to obtain data for comparison with deep ocean corrosion data.

Corrosion rates, types of corrosion and pit depths were determined.

Some highly alloyed nickel alloys, titanium alloys, silicon cast irons, specialty stainless steels, columbium, tantalum and a tantalumtungsten alloy were uncorroded in seawater both at the surface and at depth.

The corrosion rates of the copper base alloys, nickel base alloys, steels, cast irons, lead, tin, lead-tin solder, molybdenum and tungsten decreased with the concentration of oxygen in seawater, i.e., the corrosion rates were lower at depth than at the surface. The corrosion rates of $\mathrm{Ni}-200, \mathrm{Ni}-\mathrm{Cu} 402,406,410, \mathrm{~K}-500$ and $45-55, \mathrm{Ni}-\mathrm{Cr}-\mathrm{Fe} \mathrm{X} 750$, Ni-Mo2, all steels, grey cast iron and alloy cast irons decreased linearly with the concentration of oxygen in seawater.

The copper base alloys, steels, cast irons, molybdenum, tungsten, lead and lead-tin solder corroded uniformly both at the surface and at depth.

The aluminum alloys were attacked by pitting and crevice corrosion and seawater was more aggressive at depth than at the surface for such alloys. The effect of the concentration of oxygen in seawater on the corrosion of aluminum alloys was inconsistent.

The stainless steels were attacked by pitting, tunneling and crevice corrosion, except 309, 316L, 317, 329, 633, 20 Cb-3 and Ni-Cr-Mo-Si. Surface seawater was more aggressive than seawater at depth in promoting these types of corrosion on the stainless steels.

Approved for public release; distribution unlimited 
PREFACE

The Naval Civil Engineering Laboratory has been conducting a research program to determine the effects of deep ocean environments on materials. It is expected that this research will establish the best materials to be used in deep ocean construction.

A Submersible Test Unit (STU) was designed, on which many test specimens can be mounted. The STU can be lowered to the ocean floor and remain there for long periods of exposure.

Thus far, exposures have been made at two deep-ocean test sites and at a surface seawater site in the Pacific Ocean. Seven STUs have been exposed and recovered. Test Site I (nominal depth of 6,000 feet) is approximately 81 nautical miles west-southwest of Port Hueneme, California, latitude $33^{\circ} 44^{\prime} \mathrm{N}$ and longitude $120^{\circ} 45^{\prime} \mathrm{W}$. Test Site II (nominal depth of 2,500 feet) is 75 nautical miles west of Port Hueneme, California, latitude $34^{\circ} 06^{\prime} \mathrm{N}$ and longitude $120^{\circ} 42^{\prime} \mathrm{W}$. A surface seawater exposure site (V) was established at Point Mugu, California, (latitude $34^{\circ} 06^{\prime} \mathrm{N}$ and 1ongitude $119^{\circ} 07^{\prime} \mathrm{W}$ ) to obtain surface immersion data for comparison purposes.

This report presents the results of the evaluation of the different alloys exposed at the surface immersion site for periods of 12 and 18 months. 



\section{INTRODUCTION}

The development of deep diving vehicles which can stay submerged for long periods of time has focused attention on the deep ocean as an operating environment. This has created a need for information concerning the behavior of both common and potential materials of construction at depths in the ocean.

To study the problems of construction in the deep ocean, project "Deep Ocean Studies" was established. Fundamental to the design, construction and operation of structures, and their related facilities, is information with regard to the deterioration of materials in deep ocean environments. This portion of the project is concerned with determining the effects of these environments on the corrosion of metals and alloys.

In order to determine the differences between the corrosiveness of seawater at depths and at the surface it is desirable to compare deep ocean corrosion data with surface immersion data. Since surface data was not available in the literature for many of the alloys exposed at depths in the Pacific Ocean, it was decided to establish a surface exposure site to obtain this information. Therefore, a third site, designated at Site $\mathrm{V}$, was established at Point Mugu, California, latitude $34^{\circ} 06^{\prime} \mathrm{N}$ and longitude $119^{\circ} 07^{\prime} \mathrm{W}$.

The locations of the three test sites, two deep ocean sites and the surface site, are shown in Figure 1. The specific geographical locations of the test sites and the average characteristics of the seawater at these sites are given in Table 1.

Reports pertaining to the performance of alloys in the deep ocean environments are given in References 1 through 9.

This report presents a discussion of the results obtained of the corrosion of various alloys exposed at the surface, Site V, for periods of 12 and 18 months.

RESULTS AND DISCUSSIONS

The results presented and discussed herein also include the corrosion data for the alloys exposed at the surface for the International Nickel Company, Inc. Permission for their use has been granted by Dr. T. P. May, Reference 10 .

The deep ocean data for depths of 2,500 and 6,000 feet after comparable periods of exposure are included for comparison purposes. 
The chemical compositions of the aluminum alloys are given in Table 2 and their corrosion rates and types of corrosion in Table 3 . The variations of the corrosion rates and maximum pit depths of the alloys with depth and with oxygen content of seawater are shown graphically in Figures 2 through 9.

Aluminum alloys corrode chiefly by the pitting and crevice types in seawater, both of which are localized types, which means that the greater portion of the surface area of a specimen is unattacked. Therefore, corrosion rates calculated from weight losses and expressed as mils per year, which indicates uniform thinning of the material, are very misleading because they create an erroneous impression of the behavior of the material. In order to present a more realistic picture of the behavior of aluminum alloys, the maximum and average pit depths and the maximum depth of crevice corrosion are also reported.

In Figure 2 the corrosion rates of the aluminum alloys versus depth are shown. The variation of the oxygen content of seawater with depth is also shown in Figure 2. The corrosion rates of aluminum alloys 1100-H14, 5083-H113 and 3003-H14 increase progressively with depth. Those of the 6061-T6 and 2219-T81 alloys are greater at depth than at the surface but their increases are not progressive since their rates at the 2,500-foot depth are greater than those at the 6,000-foot depth. The corrosion rate of $2024-0$ at the 6,000-foot depth was greater than at the surface, but at the 2,500-foot depth it was less than at the surface. The corrosion rate of 5086-H34 decreased slightly with depth. It is shown in Figure 2 that based on corrosion rates the corrosion of 5083-H113, 1100-H14 and 3003-H14 aluminum alloys are depth dependent.

The corrosion rates of aluminum alloys 2219-T81 and 6061-T6 increased with the decreasing concentration of oxygen in seawater while those of 5086-H34 decreased slightly as shown in Figure 3.

The corrosion rates of aluminum alloys 1100-H14, 3003-H14, 2024-0 and 5083-H113 are independent of the concentration of oxygen in seawater as shown in Figure 4. The corrosion rates of three of these alloys, 1100-H14, 3003-H14 and 5083-H113, were shown to be depth (pressure) dependent, Figure 2.

The maximum depths of pits of aluminum alloys 3003-H14, 2024-0 and 5083-H113 increased with depth (pressure), i.e., they were pressure dependent as shown in Figure 5. The maximum depths of pits of alloy 5086-H34 decreased with increase in depth. Although those of alloys 2219-T81 and 6061-T6 were deeper at a depth of 6,000 feet than at the surface, the depths of pits were at the maximums at the 2,500-foot depth, Figure 5.

The maximum depths of pits of aluminum alloys 2024-0, 2219-T81 and 6061-T6 increased as the concentration of oxygen in seawater decreased, while those of 5086-H34 decreased with the concentration of oxygen, Figure 6 . 
The maximum depths of pits in aluminum alloys 3003-H14 and 5083H113 were independent of the concentration of oxygen in seawater, Figure 7. The maximum pit depths of these two alloys were depth (pressure) dependent as shown in Figure 5.

The corrosion rates of 6061-T6 and the 5000 series alloys (5083, 5086 and 5456) decreased with increasing time of exposure in surface seawater while their maximum pit depths increased with time of exposure as shown in Figure 8. The corrosion rates of alloys 3003-H14, Alclad 3003-H12 and 2219-T81 did not decrease constantly with increasing time of exposure in surface seawater; they decreased with time through 540 days of exposure and thereafter increased sharply as shown in Figure 9.

The depths of the maximum pits in alloy 2219-T81 increased with time of exposure, those in alloy 3003-H14 decreased initially and after 400 days increased rapidly, Figure 9. The depths of the maximum pits in Alclad 3003-H12 increased through the first 400 days of exposure and thereafter became constant with time. This constancy is explained by the fact that the sacrificial protective alloy layers on the Alclad 3003-H12 are corroded 1aterally, thus preventing pitting of the protected core alloy.

The corrosion rates as well as the maximum pit depths of 6061-T6 and 2219-T81 increased with decreasing concentration of oxygen in seawater, Figures 3 and 6 . However, both the corrosion rates and maximum pit depths of 5086-H34 decreased with the concentration of oxygen in seawater. Although the maximum pit depths of 2024-0 increased with decreasing concentration of oxygen in seawater, Figure 6 , its corrosion rate appears to be affected to a much lesser extent by changes in the concentration of oxygen in seawater, Figure 4. Neither the changes in the corrosion rates nor the maximum pit depths of aluminum alloys 3003H14 and 5083-H113 appear to be dependent upon the changes in the concentration of oxygen in seawater as shown in Figures 4 and 7 . They are generally greater at the lower concentrations of oxygen, although not progressively. The corrosion rates of aluminum alloys 1100-H14, 3003H14 and 5083-H113 were depth (pressure) dependent in that they increased with depth, Figure 2, while those of 5086-H34 alloy decreased slightly with increasing depth. The corrosion rates of aluminum alloys 6061-T6, 2024-0 and 2219-T81 were not consistently influenced by depth, Figure 2. The maximum pit depths of four alloys, 5083-H113, 2024-0, 5086-H34 and 3003-H14 appear to have been affected by depth; those of 5083-H113, 2024-0 and 3003-H14 increased with depth while those of 5086-H34 decreased with increasing depth, Figure 5. The maximum pit depths of alloys 2219-T81 and 6061-T6 were not consistently affected by depth except that their maximum pit depths at a depth of 6,000 feet were deeper than at the surface. In general, the corrosion rates of the aluminum alloys decreased with increasing time of exposure in surface seawater while the maximum depths of the pits increased with time of exposure, Figures 8 and 9. 
The chemical compositions of the copper alloys are given in Table 4 and their corrosion rates in Table 5. The effects of depth, concentration of oxygen in seawater and time on the corrosion rates are shown graphically in Figures 10 through 12.

Copper alloys corrode uniformly, hence corrosion rates calculated from weight losses and reported as mils per year reflect the true condition of the alloys. Therefore, corrosion rates for the copper alloys can be used reliably for design purposes. However, this does not apply to the copper base alloys which are susceptible to parting corrosion.

The variation of the corrosion rates of copper and the copper alloys with depth in the Pacific Ocean are shown in Figure 10. Since the corrosion rates of all the copper alloys, except those attacked by parting corrosion, were so comparable, the average values were plotted in Figure 10. The corrosion of copper was insensitive to depth as well as to the changes of concentration of oxygen in seawater at depth as shown in Figure 10. The oxygen concentration curve was included in Figure 10 to show its variation with depth and to show whether the corrosion rate curves were of comparable shape. The average corrosion rate curve for the copper alloys, although showing a slight decrease with depth, did not decrease gradually; hence it is more oxygen than depth dependent. The corrosion rates of only one alloy, Nickel-Silver 非752, increased gradually with increasing depth, Figure 10; hence its corrosion is mostly depth dependent.

The corrosion of copper was independent of the concentration of oxygen in seawater as shown in Figure 11. However, the corrosion of the copper alloys decreased slightly with decreasing concentration of oxygen in seawater.

The corrosion rates of copper and the copper alloys decreased with increasing time of exposure in surface seawater as shown in Figure 12.

The following alloys were attacked by parting corrosion in seawater: commercial bronze, red brass, Muntz metal, manganese bronze A and nickel-manganese bronze, containing from 10 to 42 percent zinc, were dezincified; aluminum bronzes containing 5, 7, 10, 11 and 13 percent aluminum were dealuminified.

\section{NICKEL ALLOYS}

The chemical compositions of the nickel and nickel alloys are given in Table 6 and their corrosion rates and types of corrosion in Table 7. The effects of depth, concentration of oxygen in seawater and time are shown graphically in Figures 13 to 19.

In stagnant seawater and underneath fouling many of the nickel alloys are attacked by pitting and crevice corrosion in addition to general surface attack. Under the same conditions some of the more 
highly alloyed nickel alloys are immune to corrosion, such as $\mathrm{Ni}-\mathrm{Cr}-\mathrm{Fe}$ 718, Ni-Cr-Mo 3 and 625, Ni-Mo-Cr "C", and $\mathrm{Ni}-\mathrm{Cr}-\mathrm{Fe}-\mathrm{Mo}$ " $\mathrm{F}$ ", "G" and "X". $\mathrm{Ni}-\mathrm{Co}-\mathrm{Cr}-\mathrm{Mo} 700$ alloy was attacked only by incipient crevice corrosion after 400 days of exposure at a depth of 2,500 feet.

The effect of depth on the corrosion of nickel alloys is shown in Figures 13, 14 and 15. The corrosion rates of alloys Ni-Cr-Fe 610 (cast) and 88 decreased with increasing depth, Figure 14. The corrosion rates of alloys Ni-Cu 400, Ni-Cr 75, 65-35 and 80-20, and Ni-Cr-Fe 600 and X750 decreased from the surface to the 2,500-foot depth and remained constant to the 6,000-foot depth,Figures 13, 14 and 15. A11 the other alloys except Ni-Sn-Zn 23 and Ni-Si D were more affected by the oxygen concentration than by depth. The corrosion rates of Ni-Sn-Zn 23 and Ni-Si D alloys were higher at the 6,000-foot depth than either at the surface or at the 2,500-foot depth, showing that neither depth nor oxygen were exerting the major influence on the corrosion of these two alloys.

The effect of the concentration of oxygen in seawater on the corrosion rates of nickel alloys is shown in Figures 16, 17 and 18. The corrosion rates of alloys electrolytic nickel, Ni-200, 201, 210, 211 and $301, \mathrm{Ni}-\mathrm{Cu} 402,406,410, \mathrm{~K} 500$, K505 and 45-55, Ni-Cr-Fe X750, $\mathrm{Ni}-\mathrm{Mo}-\mathrm{Fe}$ "B", $\mathrm{Ni}-\mathrm{Cr}$ 80-20, and Ni-Mo 2 decreased with decreasing concentration of oxygen in seawater as shown in Figures 16, 17 and 18 . The corrosion rates of some alloys decreased with the oxygen concentration to about $1.35 \mathrm{ml}$ per liter and thereafter remained constant to $0.4 \mathrm{ml}$ per liter - alloys $\mathrm{Ni}-\mathrm{Cu} 400, \mathrm{Ni}-\mathrm{Cr}-\mathrm{Fe} 600$ and $\mathrm{Ni}-\mathrm{Cr} 75$. The corrosion of alloys Ni-Sn-Zn 23 and Ni-SiD are apprently not affected to any major extent by the concentration of oxygen in seawater, Figures 17 and 18.

The effect of time on the corrosion rates of some nickel alloys in surface seawater is shown in Figure 19. The corrosion rates of alloys $\mathrm{Ni}-200, \mathrm{Ni}-\mathrm{Cu} 400, \mathrm{Ni}-\mathrm{Cr}-\mathrm{Fe} 600$ and $\mathrm{X750}$, and $\mathrm{Ni}-\mathrm{Fe}-\mathrm{Cr} 902$ decreased with increasing time of exposure.

In general, pitting and crevice corrosion were more rapid in surface exposure than at depth.

Welding Ni-200 with electrode No. 141 and filler metal 61 resulted in corrosion of the weld bead material and/or in the adjacent heat affected zone.

There was no accelerated corrosion of $\mathrm{Ni}-\mathrm{Cu} 400$ alloy or of the weld beads when welded with electrodes 130 and 180 ; however, weld beads of filler metal 60 and electrode 190 were attacked locally.

The corrosion of Ni-Cu $\mathrm{K} 500$ alloy was noi affected by welding with electrode 64 at the 2,500-foot depth, but the weld beads from electrodes 64 and 134 were attacked during 540 days of exposure at the surface and the weld bead of 134 electrode at the 2,500-foot depth.

The weld beads on Ni-Cr-Fe 600 alloy made from electrodes 132, 182, 62 and 82 were selectively attacked during exposure at the surface and at the 2,500-foot depth except the bead from electrode 182 at the 2,500foot depth which was only uniformly etched. 
The weld beads on $\mathrm{Ni}-\mathrm{Cr}-\mathrm{Fe} 718$ alloy made from 718 electrodes were uncorroded.

The weld beads on Ni-Cr-Fe X750 alloy made from electrodes 69 and 718 were selectively corroded during exposure at the surface and at the 2,500-foot depth, except the bead made from electrode 69 at the 2, 500-foot depth.

The weld beads on Ni-Cr-Mo 625 alloy made with 625 electrodes were uncorroded.

The weld beads on $\mathrm{Ni}-\mathrm{Fe}-\mathrm{Cr} 800$ alloy made with electrodes 82 and 138 were selectively attacked during exposure at the surface and at the 2,500-foot depth.

The weld beads on Ni-Fe-Cr 825 alloy made with electrode 135 were selectively attacked while weld beads made with electrode 65 were unattacked at the 2,500-foot depth and only by incipient pitting at the surface.

\section{STEELS}

The chemical compositions of the steels are given in Table 8 and their corrosion rates and types of corrosion in Table 9. The effects of depth, concentration of oxygen in seawater and time are shown graphically in Figures 20 to 22.

Since the corrosion rates of the steels were nearly the same at any one depth, the average values for any one depth were averaged and plotted in Figures 20 to 22.

The effect of depth on the average corrosion rate of the steels is shown in Figure 20. The variation of the concentration of oxygen in seawater with depth is also plotted in Figure 20 for comparison purposes. The shapes of the curves for the steels and AISI 1010 steel show that the corrosion rates are not depth (pressure) dependent. The shapes of those curves are practically the same as the shape of the oxygen curve, indicating that the concentration of oxygen exerts a major influence on the corrosion of steels in seawater.

The effect of the concentration of oxygen in seawater on the corrosion rates of steels is shown in Figure 21. The curve for the average corrosion rates of all the steels is a straight line, indicating that the corrosion rates of steels in seawater are proportional to the oxygen concentration.

The corrosion rate of AISI 1010 steel and the averages of the corrosion rates of all the carbon and low alloy steels after one year of exposure versus the oxygen content and the temperature of seawater were analyzed using the technique of linear regression analysis. By this technique a relationship between oxygen content, temperature and corrosion rate was obtained for both the average of all carbon and low alloy steels and for AISI 1010 steel. The derived formulae are: 


$$
\begin{aligned}
& \text { Corrosion Rate }(\mathrm{MPY})=0.84+1.0\left(\mathrm{O}_{2}\right)+0.014 \text { (T) } \\
& \quad \text { (avg of carbon and } \\
& \quad \text { low alloy steels) } \\
& \text { Corrosion Rate (MPY) }=0.19+1.1\left(\mathrm{O}_{2}\right)+0.1(\mathrm{~T})
\end{aligned}
$$$$
\text { (AISI 1010) }
$$

The corrosion rates are in mils per year (MPY), the oxygen content of seawater in milliliters per liter $(\mathrm{ml} / 1)$ and temperature in degrees Centigrade $\left({ }^{\circ} \mathrm{C}\right)$.

These derived formulae illustrate two important points:

(1) The concentration of oxygen in seawater is a major variable and its effect on the corrosion rate of steel in seawater is linear.

(2) The temperature of the seawater has less effect on the corrosion of steel in seawater than the oxygen content and its effect is also linear.

These formulae, however, cannot be used to predict the corrosion rates of steels in seawater at other locations due to the influences of other variables such as time, currents, sediment effects, etc. For example, the above formulae are not satisfactory for predicting corrosion rates for steels in the Tongue-of-the-0cean, Atlantic Ocean. Since they are not applicable, it is obvious that other variables in that location are different from those in the Pacific Ocean off the Channel Islands.

The effect of time of exposure in surface seawater on the average corrosion rates of steels is shown in Figure 22. The corrosion rates decrease parabolically with increasing time of exposure.

All the steels except AISI Type 502, in general, corroded uniformly except for some pitting in surface seawater which was caused by fouling. AISI Type 502, because it contained about 5 percent chromium, was pitted.

\section{CAST IRONS}

The chemical compositions of the cast irons are given in Table 10 and their corrosion rates and types of corrosion in Table 11. The effects of depth, concentration of oxygen in seawater and time are shown graphically in Figures 23 to 25.

The effect of depth on the corrosion rates of the cast irons is shown in Figure 23. The shape of the corrosion rate curve for the alloy 
cast irons was very close to that of the oxygen curve and shows that the corrosion of the alloy cast irons is not depth dependent. The shapes of the curves for gray cast iron, the austenitic cast irons, and the silicon and silicon-molybdenum cast irons show that depth is not an important variable in their corrosion behavior.

The effect of the concentration of oxygen in seawater on the corrosion rates of cast irons is shown in Figure 24. The corrosion rates of gray cast iron and the alloy cast irons decreased practically linearly with the concentration of oxygen in seawater. The corrosion rates of the austenitic cast irons decreased with the concentration of oxygen in seawater while the silicon and silicon-molybdenum cast irons were uncorroded; hence were insensitive to the concentration of oxygen.

All the cast irons corroded uniformly except the silicon and silicon-molybdenum cast irons which were uncorroded.

The effect of time of exposure on the corrosion of cast irons during surface exposure in seawater is shown in Figure 25. Data were available for only two austenitic cast irons and their corrosion rates decreased asymptotically with increasing time of exposure. Their corrosion rates became practically constant at between 2 and 3 mils per year after about two years of exposure.

\section{STAINLESS STEELS}

The chemical compositions of the stainless steels are given in Table 12 and their corrosion rates and types of corrosion in Tables 13 through 17. The effect of depth and the concentration of oxygen in seawater on the corrosion rates of stainless steels are shown graphically in Figures 26 through 31.

In general, stainless steels corrode chiefly by pitting and crevice corrosion in seawater. In these types of localized attack the majority of the surface area is unattacked so that corrosion rates calculated from weight losses are very misleading because they reflect a uniform thinning of the material. However, in spite of this, the corrosion rates of a number of the stainless steels were plotted versus depth and the concentration of oxygen in seawater to see if any information of value could be obtained.

The corrosion rates of the 200 and 400 Series stainless steels as affected by depth are shown in Figure 26. The corrosion rates of AISI 430 and $18 \mathrm{Cr}-14 \mathrm{Mn}-0.5 \mathrm{~N}$ stainless steels decreased with increasing depth. The corrosion rates of AISI 201, 202, 410 and 446 were lower at depth than at the surface, but they did not decrease progressively with increasing depth.

The effects of changes in the oxygen concentration of seawater on the corrosion rates of the 200 and 400 Series stainless steels are shown in Figure 27. The corrosion rates of AISI 410 decreased linearly with the oxygen content while those for AISI 201, 202 and 446 were not 
uniformly decreased. The corrosion rates of AISI 430 and $18 \mathrm{Cr}-14 \mathrm{Mn}-$ $0.5 \mathrm{~N}$ stainless steels, although lower at the lower oxygen concentrations than at the highest oxygen concentration, were not uniformly affected by the oxygen concentration.

Examination of the pitting, tunneling and crevice corrosion data for these stainless steels in Tables 13, 15 and 17 shows only a general relationship with corrosion rates. These types of corrosion were, in general, more severe or just as severe in the surface seawater (highest oxygen concentration) than at depths of 2,500 and 6,000 feet. However, it is more realistic to assess the performance of these stainless steels on their localized types of corrosion performance than upon calculated corrosion rates.

The corrosion rates of the 300 Series stainless steels as affected by depth are shown in Figure 28. Only the corrosion rates of the AISI 304 and 304L stainless steels decreased with increasing depth. The corrosion rates of AISI 301, 302, 316, 316 (sensitized), 330, 347, 304 (sensitized) and 325 stainless steels were lower at depth than at the surface, but they did not decrease progressively with increasing depth. In addition, the shape of the corrosion rate curve for AISI 325 was similar to the oxygen concentration curve.

The effect of changes in the concentration of oxygen in seawater on the corrosion rates of the 300 Series stainless steels are shown in Figure 29. The corrosion rates of the alloys shown in Figure 29 decreased with decreasing oxygen concentration, although not uniformly.

Examination of the pitting, tunneling and crevice types of corrosion in Table 14 for the alloys whose corrosion rates were plotted in Figures 28 and 29 shows that, in general, there is no definite correlation between their corrosion rates and the severity of these types of corrosion. For example, the corrosion rates of AISI 304L varied from 1.0 to 0.4 to $<0.1 \mathrm{MPY}$ at the three depths, while pitting corrosion was to perforation ( $115 \mathrm{mils}$ ) in all exposures while crevice and tunneling corrosion was more severe at the 6,000-foot depth where the corrosion rate was the lowest ( $<0.1 \mathrm{MPY})$.

Oxygen and depth apparently had no effect on the corrosion of the following 300 Series stainless steels: AISI 309, 310, 311, 316L, 317 , 321 (slightly affected) and 329.

The effect of depth on the corrosion rates of some of the 600 Series precipitation hardening stainless steels is shown in Figure 30 . The corrosion rate of $631-$ TH1050 and 635 decreased with increasing depth of seawater. The corrosion rates of $630-\mathrm{H} 925$ and $632-\mathrm{RH} 1100$ were lower at depth than at the surface but they did not decrease progressively with increasing depth.

The effect of changes in the concentration of oxygen in seawater on the corrosion rates of the 600 Series precipitation hardening stainless steels is shown in Figure 31. The corrosion rate of AISI 632RH1100 decreased progressively with the oxygen content of seawater. The corrosion rates of AISI 630-H925, 631-TH1050 and 635, although 
lower at the lower oxygen concentrations than at the highest, did not decrease progressively with the oxygen concentration.

Here again, comparison of the corrosion rates with the severity of the pitting, tunneling and crevice types of corrosion (Table 6) showed no definite correlations.

The corrosion rates and types of corrosion of the miscellaneous cast and wrought stainless steels are given in Table 17. Except for the $18 \mathrm{Cr}-14 \mathrm{Mn}-0.5 \mathrm{~N}$ which contained no nickel, the others contained greater percentages of chromium and nickel than the conventional stainless steels in addition to molybdenum and copper. The corrosion rates of these stainless steels were mostly less than 0.1 MPY and instances of pitting and crevice corrosion were few except for the $18 \mathrm{Cr}-15 \mathrm{Mn}-0.5 \mathrm{~N}$ alloy. Significant pitting and crevice corrosion occurred during surface exposures of wrought alloy $20-\mathrm{Cb}$ and cast alloy $\mathrm{Ni}-\mathrm{Cr}-\mathrm{Cu}-\mathrm{Mo}$ 非 .

\section{TITANIUM ALLOYS}

The chemical compositions of the titanium alloys are given in Table 18 and their corrosion rates and types of corrosion in Table 19.

There was no corrosion of any of the alloys except the welded 13V-11Cr-3AI alloy. It was susceptible to stress corrosion cracking during surface exposures. Specimens were in two welded conditions, half were butt welded and a 3-inch diameter circular weld bead was placed on the other half of the specimens. The welded specimens were intentionally not stress relieved in order to retain the maximum internal welding stresses in the specimens during exposure. The stress corrosion cracks extended across the butt welds normal to the direction of the beads and developed within 398 days of exposure. The stress corrosion cracks in the specimens with the circular welds extended radially across the weld beads and they also developed within 398 days of exposure.

\section{MISCELLANEOUS ALLOYS}

The chemical compositions of the miscellaneous alloys are given in Table 20 and their corrosion rates and types of corrosion in Table 21. The effect of depth, concentration of oxygen in seawater and time are shown in Figures 32 to 34.

Columbium, tantalum and tantalum alloy Ta60 were uncorroded during 763 days of exposure at the surface and 402 days of exposure at a depth of 2,500 feet.

The effect of depth on the corrosion rates of the miscellaneous alloys is shown in Figure 32. The corrosion rates of tin, molybdenum and tungsten decreased with increasing depth. The corrosion rates of lead and lead-tin solder were lower at depth than at the surface but 
did not decrease progressively with increasing depth. The corrosion rate of zinc, on the other hand, was much greater at the 6,000-foot depth than at either the surface or the 2,500-foot depth.

The effect of the concentration of oxygen in seawater on the corrosion rates of the miscellaneous alloys is shown in Figure 33. The corrosion rates of lead, tin, lead-tin solder, molybdenum and tungsten were lower at the lower oxygen concentrations than at the highest, but the decreases were not linear. Since there were only two points for the molybdenum and tungsten curves, there is no assurance that the curves would be linear with more points at intermediate oxygen concentrations. The corrosion rate for zinc was definitely not dependent upon the oxygen concentration of seawater; it was the same at the lowest as at the highest concentration of oxygen in seawater and twice as high at the intermediate oxygen concentration.

The effect of time of exposure at the surface on the corrosion rate of molybdenum and tungsten are shown in Figure 34. The corrosion rate of molybdenum decreased with increasing time of exposure while that of tungsten definitely increased.

\section{SUMMARY}

The purpose of this investigation was to determine the effects of surface seawater on the corrosion of different types of alloys for comparison with their deep ocean corrosion behavior. To accomplish this 1,134 specimens of 189 different alloys were exposed 5 feet below the lowest tide level in the Pacific Ocean at Point Mugu, California (Site V, Figure I) for from 366 to 763 days.

\section{Aluminum Alloys}

In general the corrosion rates of the aluminum alloys were greater at depth than at the surface in the Pacific Ocean after one year of exposure, except for 5086-H34 whose corrosion rate was slightly lower.

The maximum pit depths of the aluminum alloys were greater at depth than at the surface, except for 5086-H34 whose maximum pit depths were less than at the surface.

The corrosion rate of 5086-H34 decreased slightly with the oxygen concentration of seawater, those of 2219-T81 and 6061-T6 increased with decreasing oxygen concentration and those of 1100-H14, 5083-H113 and 3003-H14 were higher at the lower oxygen concentrations, but not progressively. The corrosion rate of 2024-0 appears to be independent of the oxygen concentration of seawater.

The maximum pit depths of alloys 2024-0, 2219-T81 and 6061-T6 increased with decreasing concentration of oxygen in seawater, while those of 5086--H34 decreased with the oxygen concentration. The maximum pit depths of 3003-H14 were deeper at the lower oxygen concentrations, but 
not progressively. The maximum pit depths of 5083-H113 were apparently not dependent upon the oxygen concentration.

The corrosion rates of the 5000 Series aluminum alloys and 6061-T6 decreased with increasing time of exposure at the surface in the Pacific Ocean while their maximum pit depths increased. The corrosion rates of 2219-T81, 3003-H14 and Alclad 3003-H12 decreased with time of exposure at the surface through 540 days of exposure and thereafter, for some unknown reason, increased rapidly. Their maximum pit depths, in general, increased with time of exposure.

The aluminum alloys were attacked by pitting and crevice types of corrosion; hence, corrosion rates calculated from weight losses are unsuitable for assessing the corrosion behavior.

Crevice corrosion, in general, was more severe at depth than at the surface.

Copper Alloys

The copper alloys, in general, corroded uniformly except for some isolated cases of pitting and cratering. Also, there was dezincification of Muntz metal and nickel-manganese bronze and dealuminification of the aluminum bronzes.

The corrosion rate of copper was essentially unaffected by depth and that of all the copper alloys was lower at depth than at the surface, but not progressively.

The corrosion rate of copper was unaffected by changes in the concentration of oxygen in seawater while the average rate of the copper alloys decreased with decreasing concentration of oxygen. The corrosion rate of Muntz metal, which also was dezincified, also decreased with the concentration of oxygen in seawater.

The corrosion rates of all the copper alloys decreased with increasing time of exposure at the surface except Muntz metal whose corrosion rate increased with time.

Nickel Alloys

Fourteen (14) of the nickel base alloys were uncorroded: Ni-CrFe 718, Ni-Cr-Mo 3, Ni-Cr-Mo 625, Ni-Fe-Cr 800, $\mathrm{Ni}-\mathrm{Fe}-\mathrm{Cr} 804$, $\mathrm{Ni}-\mathrm{Fe}-\mathrm{Cr}$ 825, Ni-Fe-Cr 825 (sensitized), Ni-Fe-Cr 825Cb, Ni-Fe-Cr 901, Ni-Cr$\mathrm{Fe}-\mathrm{Mo}$ "F", Ni-Cr-Fe-Mo "G", Ni-Cr-Fe-Mo "X", Ni-Mo-Fe "B", and Ni-Mo$\mathrm{Cr}$ "C".

The corrosion rates of the other nickel base alloys were higher at the surface than at depth. The corrosion rates of $\mathrm{Ni}-\mathrm{Cr}-\mathrm{Fe} 600$ and $\mathrm{Ni}-$ $\mathrm{Cr}-\mathrm{Fe} 88$ decreased with increasing depth while those of the other alloys did not decrease progressively with depth.

Most of the alloys which were corroded were also attacked by crevice corrosion.

The corrosion rates of all except two nickel base alloys (Ni-SnZn 23 and Ni-Si D) decreased with decreasing concentration of oxygen in 
seawater. The corrosion rates of $\mathrm{Ni}-\mathrm{Cr}-\mathrm{Fe} \mathrm{X} 750, \mathrm{Ni}-\mathrm{Mo} 2, \mathrm{Ni}-200$ and $\mathrm{Ni}-\mathrm{Cu} 402,406,410, \mathrm{~K} 500$, K505 and 45-55 alloys decreased linearly with the concentration of oxygen in seawater.

The corrosion rates of $\mathrm{Ni}-200, \mathrm{Ni}-\mathrm{Cu} 400, \mathrm{Ni}-\mathrm{Cr}-\mathrm{Fe} 600$ and $\mathrm{X} 750$, and $\mathrm{Ni}-\mathrm{Fe}-\mathrm{Cr} 902$ decreased with increasing time of exposure at the surface.

In general, pitting and crevice corrosion were more rapid in surface seawater exposure than at depth.

There was either no corrosion or uniform corrosion of weld beads and in the adjacent heat affected zones when $\mathrm{Ni}-\mathrm{Cu} 400$ alloy was welded with electrodes 130 and 180, Ni-Cr-Fe 718 with electrode 718, and NiCr-Mo 625 with electrode 625.

There was selective corrosion, line corrosion or pitting of either the weld beads or in the adjacent heat affected zones or both when Ni200 was welded with electrodes 61 and 141, Ni-Cu 400 with electrodes 60 and 190, Ni-Cu K500 with electrodes 64 and 134, Ni-Cr-Fe 600 with electrodes 62, 82, 132 and 182, Ni-Cr-Fe X750 with electrodes 69 and 718, $\mathrm{Ni}-\mathrm{Fe}-\mathrm{Cr} 800$ with electrodes 82 and 138, and $\mathrm{Ni}-\mathrm{Fe}-\mathrm{Cr} 825$ with electrodes 65 and 135 .

Steels

The steels were all corroded uniformly and their corrosion rates were comparable - carbon steels, low alloy-high strength steels, nickel steels, and the very high strength steels.

The corrosion rates of the steels were lower at depth than at the surface, but they did not decrease progressively with increasing depth; i.e., they were not depth dependent.

The average corrosion rates of all the steels decreased linearly with the concentration of oxygen in seawater.

The corrosion rates, the oxygen concentration and temperature of seawater were analyzed using linear regression analysis. The following relationships were obtained for AISI 1010 steel and the averages of the other steels:

Corrosion Rate $(\mathrm{MPY})=0.84+1.0\left(0_{2}\right)+0.014(\mathrm{~T})$

(Avg of carbon and

low alloy steels)

Corrosion Rate $(\mathrm{MPY})=0.19+1.1\left(0_{2}\right)+0.1$ (T)

(AISI 1010)

The corrosion rates are in mils per year (MPY), the oxygen content of seawater in milliliters per liter (m1/1) and temperature in degrees Centigrade $\left({ }^{\circ} \mathrm{C}\right)$. 
These derived formulae illustrate two important points:

(1) The concentration of oxygen in seawater is a major variable and its effect on the corrosion rate of steel in seawater is linear.

(2) The temperature of seawater has less effect on the corrosion of steel in seawater than the oxygen content and its effect is also linear.

These formulae, however, cannot be used to predict the corrosion rates of steels in seawater at other locations due to the influence of other variables such as time, currents, sediment effects, etc.

The corrosion rates of the steels decreased progressively with increasing time of exposure in surface seawater.

Cast Irons

Silicon and silicon-molybdenum cast irons were uncorroded in seawater at the surface and at depth in the Pacific Ocean after one year of exposure.

The corrosion rates of the other cast irons were lower at depth than at the surface, but were not depth dependent.

The corrosion rates of the alloy cast irons and gray cast iron decreased linearly with the concentration of oxygen in seawater and those of the austenitic cast irons progressively.

The corrosion rates of two austenitic cast irons, Type 4 and Type D-2C, decreased asymptotically with time of exposure at the surface in seawater.

Stainless Steels

The following stainless steels were attacked only by incipient crevice corrosion after one year of exposure in seawater: AISI Type $309,316 \mathrm{~L}, 317,329$ and $633,20 \mathrm{Cb} 3$ and $\mathrm{Ni}-\mathrm{Cr}-\mathrm{Mo}-\mathrm{Si}$.

All the other stainless steels were attacked by pitting, tunneling and crevice corrosion in various degrees of severity.

In general, the miscellaneous wrought and cast stainless steels, except the $18 \mathrm{C}-14 \mathrm{Mn}-0.5 \mathrm{~N}$ steel, were less severely attacked than the others.

Titanium Alloys

The titanium alloys, unwelded and welded, except the 13V-11Cr-3Al alloy, were uncorroded. Welded $13 \mathrm{~V}-11 \mathrm{Cr}-3 \mathrm{Al}$ titanium alloy was susceptible to stress corrosion cracking when the welding stresses were not relieved by thermal treatment. 
Columbium, tantalum and tantalum-tungsten alloy Ta60 were uncorroded. However, magnesium alloy FS-1 was practically disintegrated after one year of exposure in seawater.

The corrosion of lead (antimonial chemical and tellurium), tin, zinc, lead-tin solder, molybdenum and tungsten were not depth dependent.

The corrosion rates of lead, tin, lead-tin solder, molybdenum and tungsten decreased with the concentration of oxygen in seawater while that of zinc was not dependent on the oxygen concentration.

The corrosion rate of molybdenum decreased with increasing time of exposure in seawater at the surface while that of tungsten increased.

\section{CONCLUSIONS}

Seawater at depth in the Pacific Ocean at the NCEL test sites was more aggressive to aluminum alloys than was seawater at the surface after one year of exposure, except for 5086-H34 alloy whose corrosion rate was slightly lower at depth.

In general, the corrosion rates and maximum pit depths of the aluminum alloys increased with decreasing oxygen concentration of seawater.

Aluminum alloys, because their modes of corrosion are the localized pitting and crevice types, must be protected for seawater applications if reasonable service life is desired. In general, aluminum alloys could not be recommended for deep sea applications for periods longer than three years if protective maintenance cannot be performed.

In most cases the copper base alloys corroded either at the same " rates or slightly slower rates at depth than at the surface in seawater. Copper base alloys which are susceptible to dezincification and dealuminification are not recommended for seawater service. The other copper alloys corroded uniformly and can be recommended for seawater service where their low corrosion rates can be tolerated.

The nickel base alloys which were not corroded in seawater can be recommended for seawater applications.

Nickel base alloys susceptible to crevice corrosion are not recommended for seawater applications unless satisfactory precautions can be taken to prevent this type of attack.

The use of welded nickel alloys for seawater applications can be recommended only for those alloys which are not preferentially attacked in either the weld beads or the adjacent heat affected zones or both.

Steels and cast irons, because they corrode uniformly, can be recommended for seawater applications and their reliability can be increased by the use of adequate protective measures.

The stainless steels, because of their susceptibility to crevice, pitting and tunnel corrosion, are not recommended for seawater applications. Alloys 309, 316L, 317, 329, 633, 20Cb-3 and Ni-Cr-Mo-Si could be used for limited applications of not more than one year if adequate protective measures are used. 
Titanium alloys, except welded $13 \mathrm{~V}-11 \mathrm{Cr}-3 \mathrm{Al}$ alloy, are recommended for seawater applications.

Columbium, tantalum and tantalum alloy Ta60 are recommended for seawater service where the expense can be justified.

Magnesium alloy FS-1 is unsatisfactory for seawater applications.

Molybdenum, tungsten and lead (chemical, antimonial and tellurium), because of their low uniform corrosion, can be recommended for seawater applications where their mechanical and physical properties fulfill the requirements.

Tin, zinc and lead-tin solder are not recommended for seawater service. Zinc of special purity, however, is used as sacrificial anodes to protect more noble alloys in many seawater applications. 


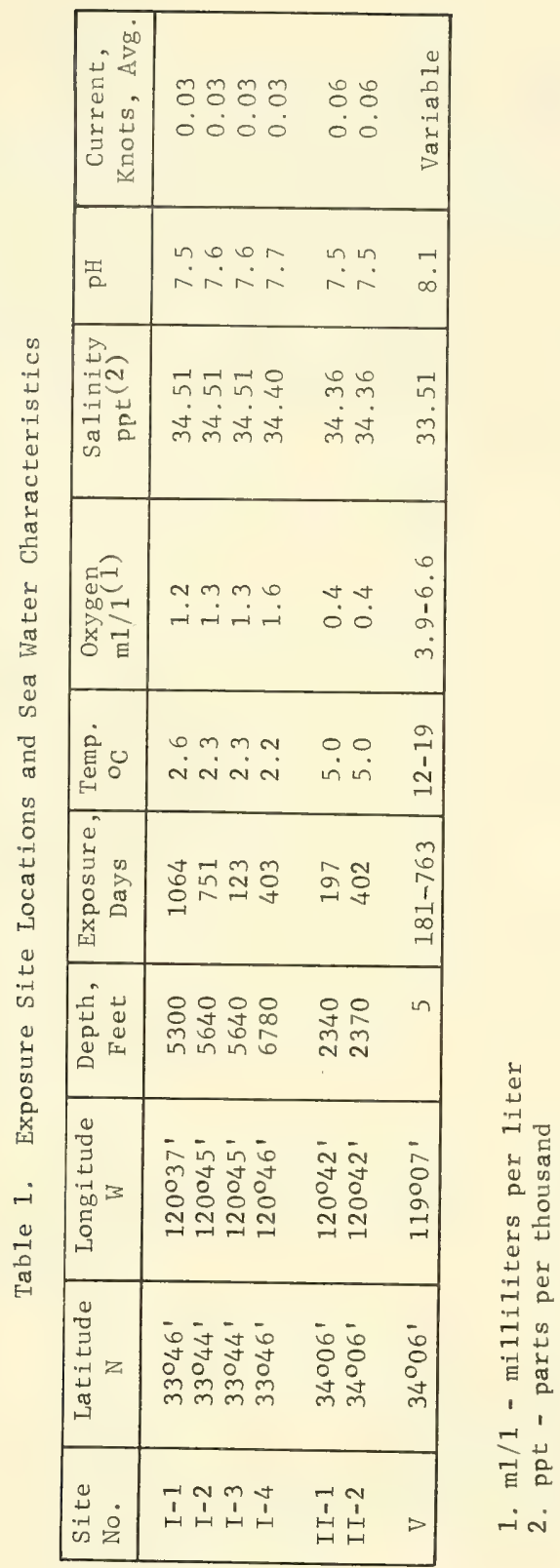




\begin{tabular}{|c|c|c|c|c|c|c|}
\hline 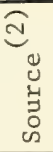 & 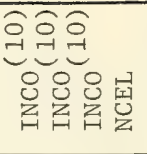 & 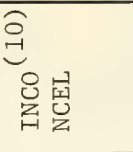 & 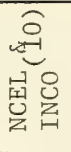 & 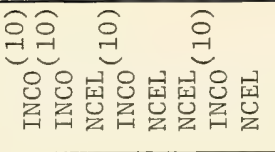 & 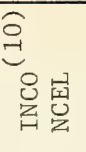 & 四 \\
\hline ন & 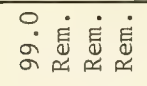 & 运离 & 离 & 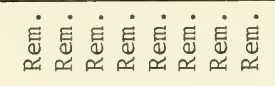 & 它悹 & $\underset{\dot{E}}{\dot{\Xi}}$ \\
\hline$\ddot{H}$ & $\begin{array}{ll}1 & 0 \\
1 & 0 \\
0\end{array}$ & 11 & 11 & 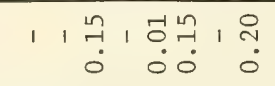 & $1 \stackrel{\operatorname{Ln}}{7}$ & $\stackrel{0}{\circ}$ \\
\hline 品 & $\begin{array}{llll}1 & 1 & 1 & 0 \\
& & & 0 \\
0\end{array}$ & $\begin{array}{l}\text { nी } \\
0 \\
0 \\
0 \\
0\end{array}$ & $\begin{array}{l}0 \\
\stackrel{-1}{-1} \\
0 \\
0\end{array}$ & 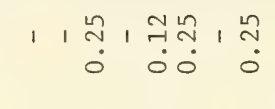 & $1 \stackrel{\Perp n}{0}$ & $\stackrel{0}{i}$ \\
\hline$\stackrel{-1}{z}$ & 111 & $\begin{array}{l}10 \\
0\end{array}$ & 11 & $\begin{array}{llllllll}1 & 1 & 1 & 1 & 1 & 1 & 1 & 1\end{array}$ & 11 & 1 \\
\hline Uै & $\begin{array}{llll}1 & 1 & 1 & 1\end{array}$ & $\begin{array}{l}10 \\
0 \\
0\end{array}$ & 11 & 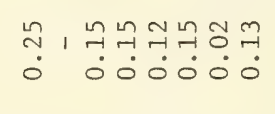 & 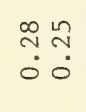 & 웅 \\
\hline$\sum^{\infty}$ & $\begin{array}{l}1 \ln \ln \\
-1-i 0\end{array}$ & $\begin{array}{l}\dot{0} \\
\dot{0} \\
\dot{2}\end{array}$ & $1 \stackrel{0}{+}$ & 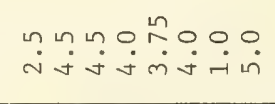 & $\begin{array}{l}0 \\
-i \\
-i\end{array}$ & $\stackrel{\infty}{\sim}$ \\
\hline$\stackrel{E}{\Sigma}$ & 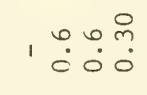 & 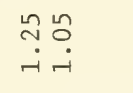 & 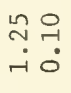 & 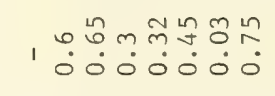 & 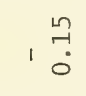 & $\stackrel{1}{2}$ \\
\hline$\vec{U}$ & 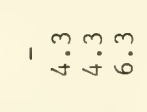 & In & $\stackrel{\circ}{\circ} \stackrel{0}{\because}$ & 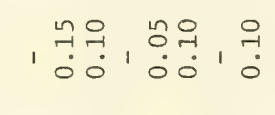 & ๗ั๊ & $\stackrel{0}{\rightarrow-1}$ \\
\hline 记 & 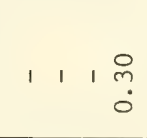 & \begin{tabular}{l} 
in $\infty$ \\
\multirow{1}{*}{$: 0$} \\
0 \\
0
\end{tabular} & 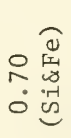 & 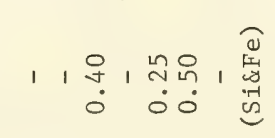 & $1 \stackrel{0}{0}$ & 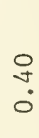 \\
\hline is & $\begin{array}{lll}1 & 1 & 0 \\
\end{array}$ & 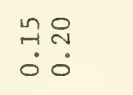 & $\begin{array}{l}\circ 0 \\
0 \\
0 \\
0\end{array}$ & 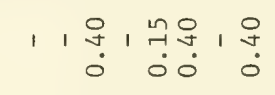 & $\begin{array}{l}1 \\
1 \\
0 \\
0\end{array}$ & ? \\
\hline 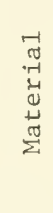 & 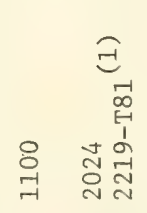 & 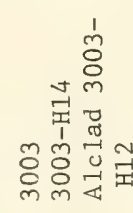 & 点 & 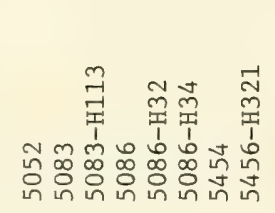 & 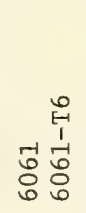 & 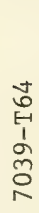 \\
\hline
\end{tabular}




\begin{tabular}{|c|c|}
\hline 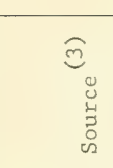 & 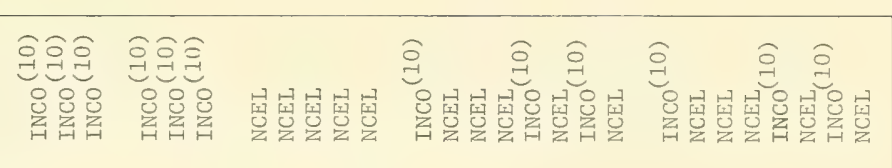 \\
\hline 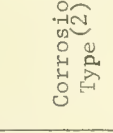 & 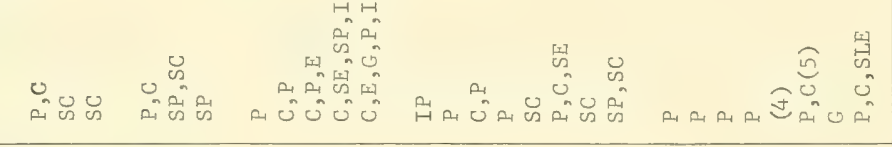 \\
\hline $\begin{array}{ll}0 \\
6\end{array}$ & 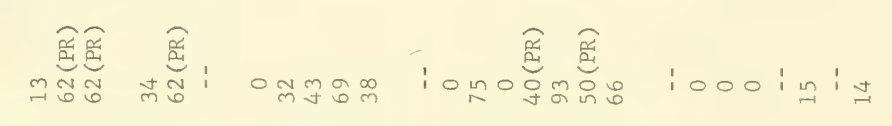 \\
\hline 要 & 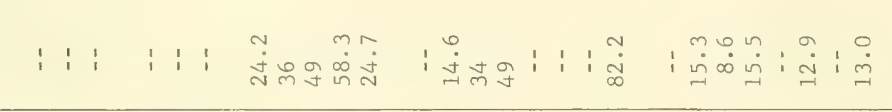 \\
\hline 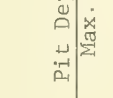 & 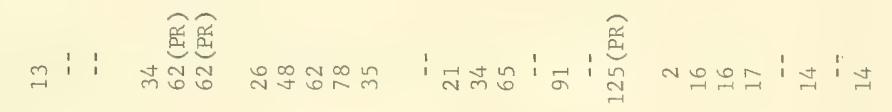 \\
\hline $\begin{array}{llll} & \end{array}$ & 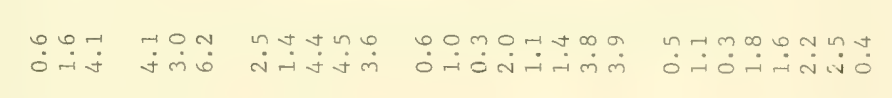 \\
\hline 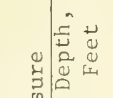 & 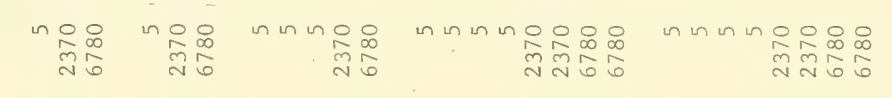 \\
\hline 总㩊 & 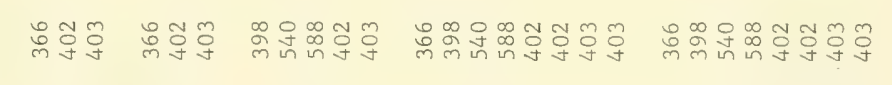 \\
\hline 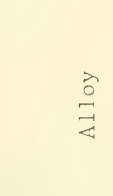 & 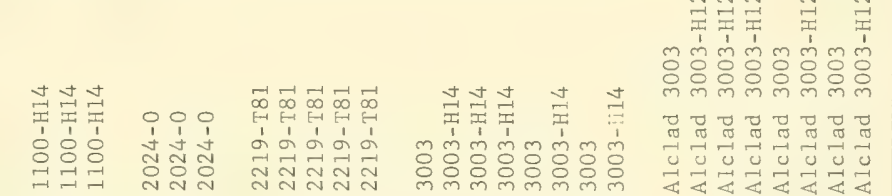 \\
\hline
\end{tabular}




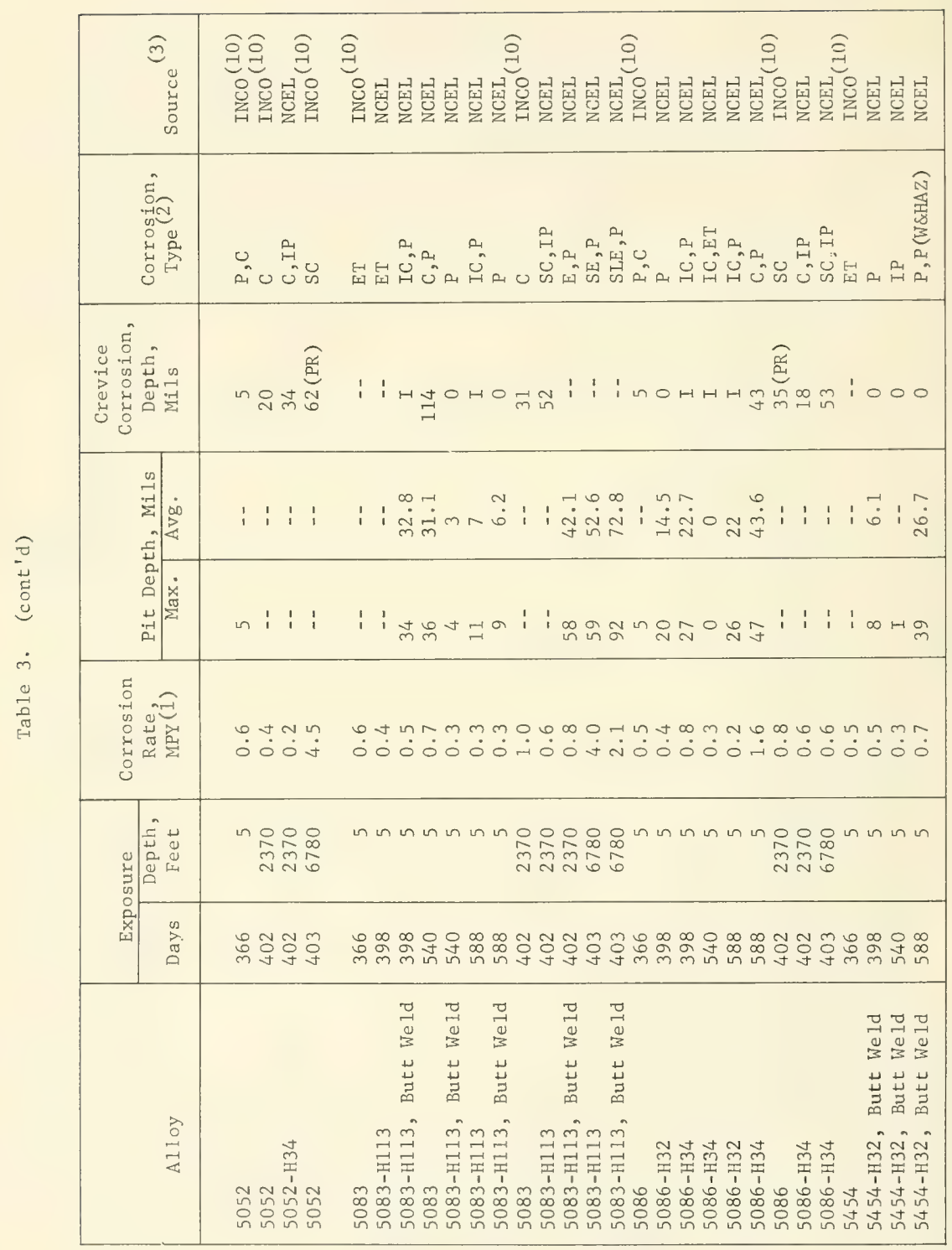




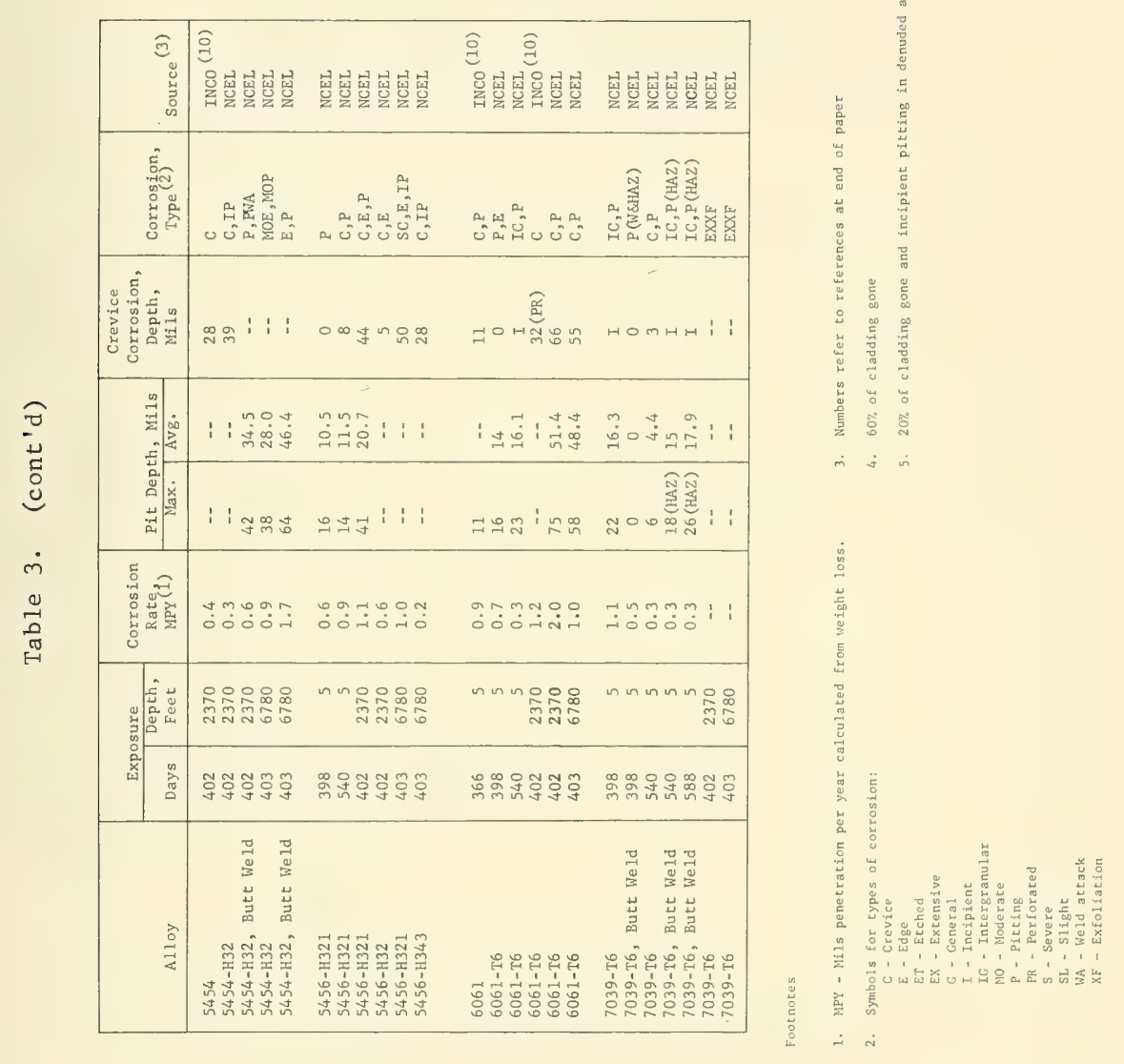




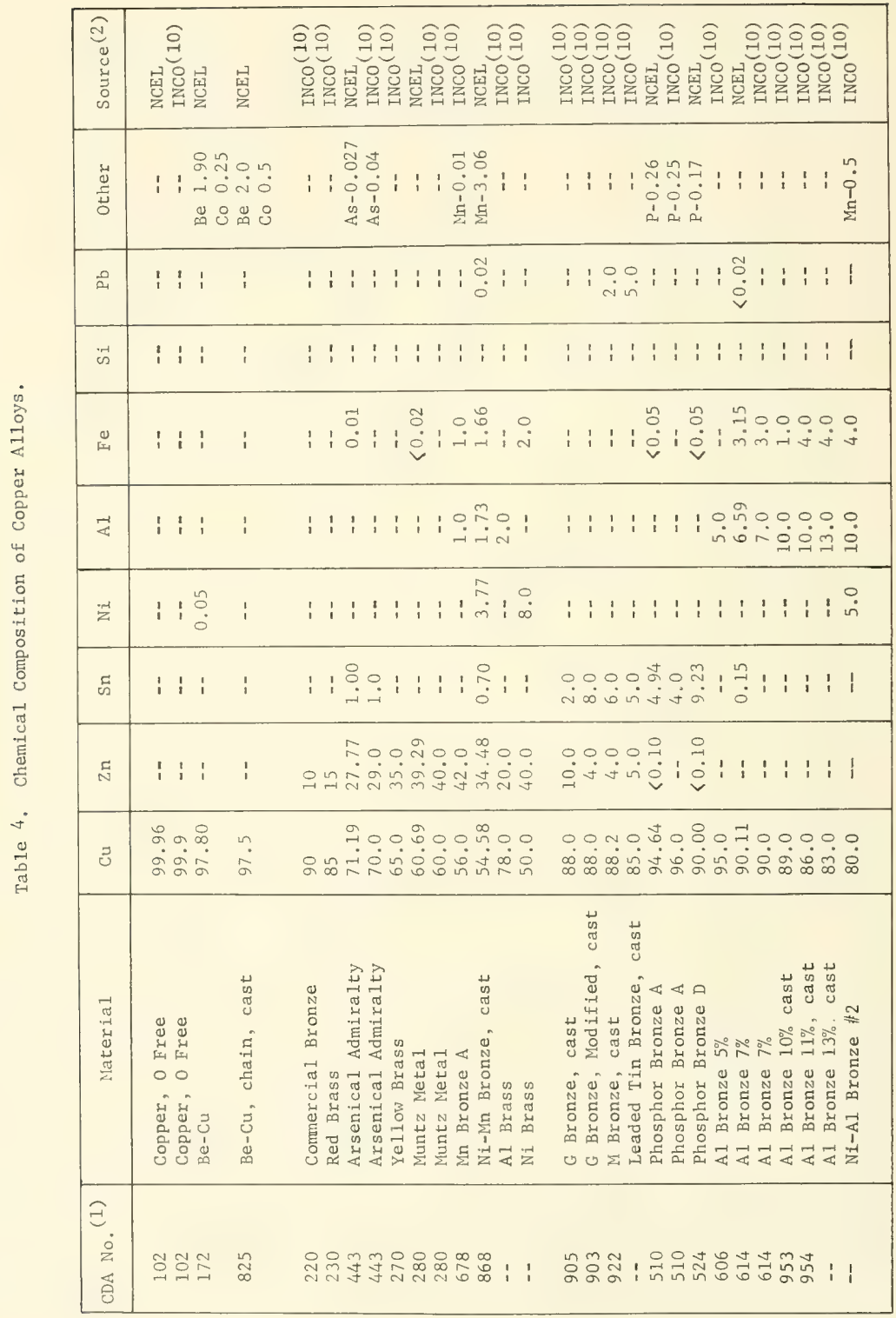




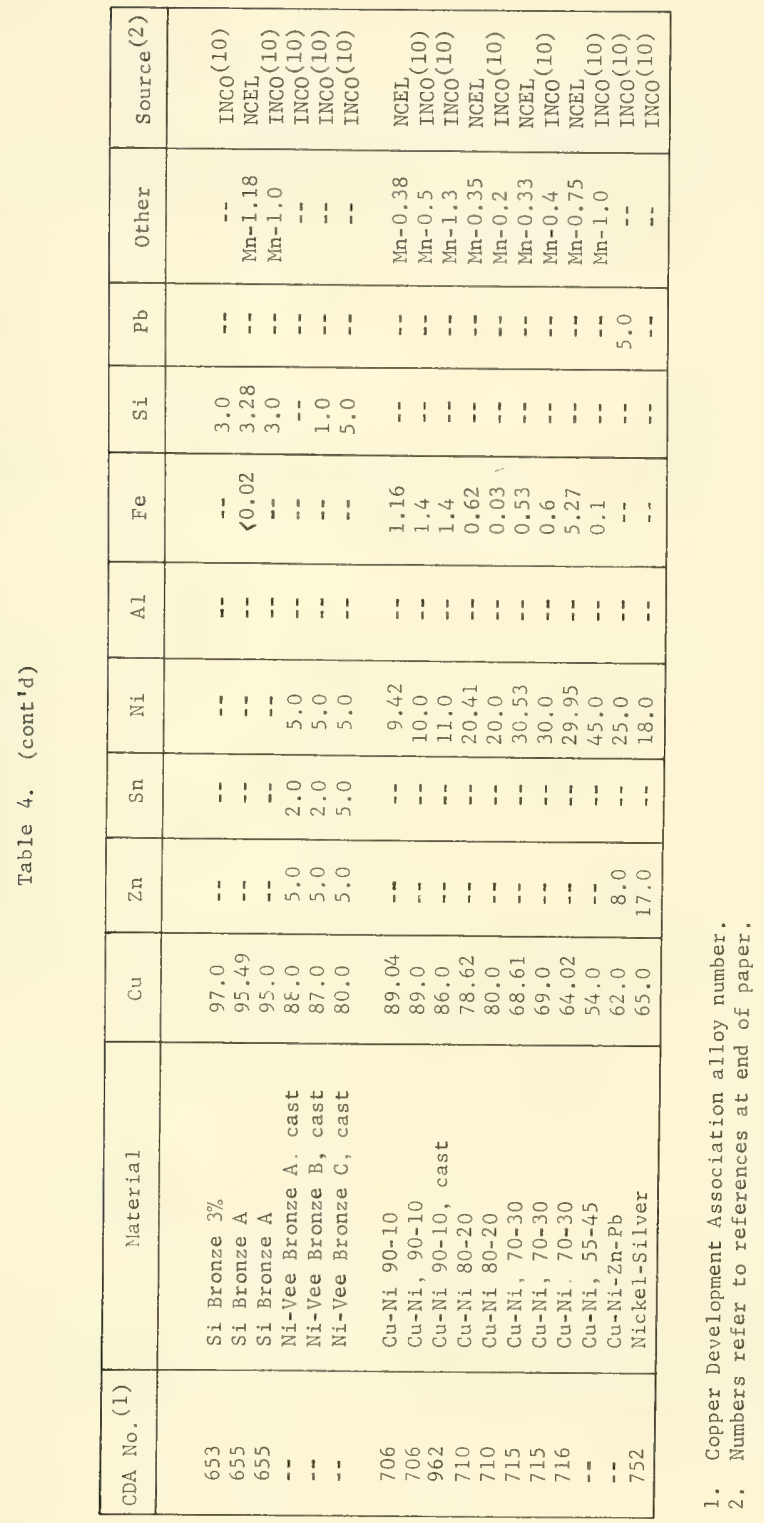




\begin{tabular}{|c|c|c|c|c|}
\hline 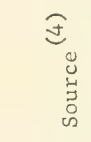 & 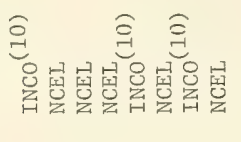 & 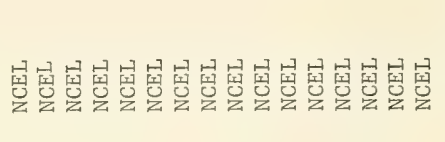 & 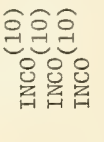 & 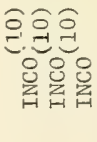 \\
\hline 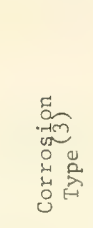 & 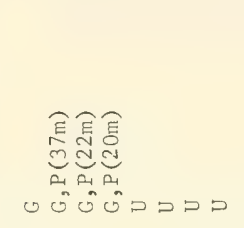 & 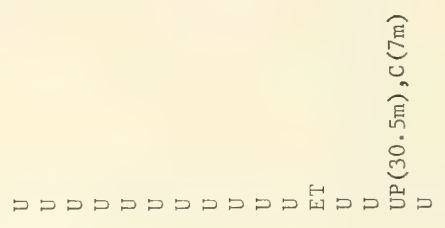 & 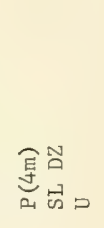 & 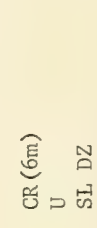 \\
\hline 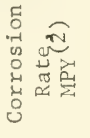 & 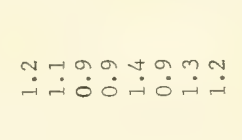 & 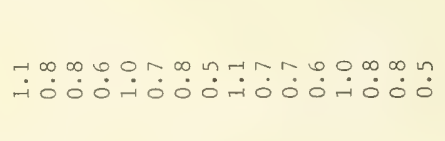 & $\dddot{r} \mathfrak{m}$ & $\stackrel{\sim}{\sim} \div$ \\
\hline 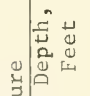 & 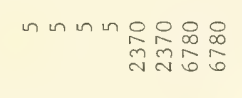 & 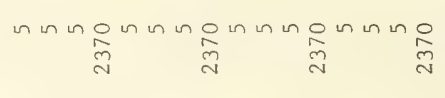 & $\begin{array}{l}n \circ \infty \\
\text { ñ } \\
\text { Nio }\end{array}$ & n \\
\hline$\stackrel{\substack{n \\
\stackrel{\pi}{\sigma}}}{\stackrel{\infty}{\sigma}}$ & 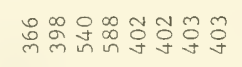 & 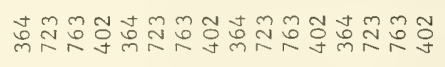 & 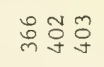 & 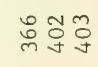 \\
\hline 至。 & 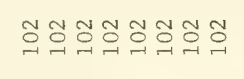 & 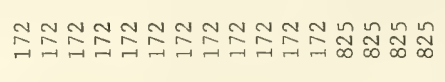 & ลิ กิ & 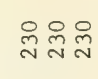 \\
\hline 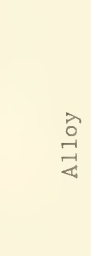 & 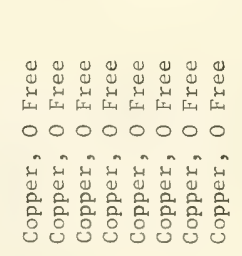 & 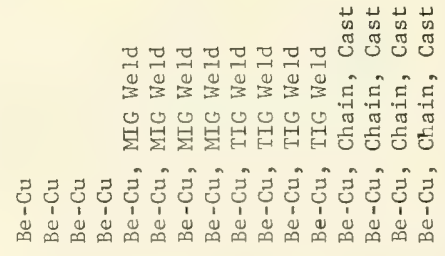 & 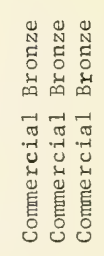 & 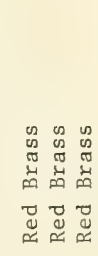 \\
\hline
\end{tabular}




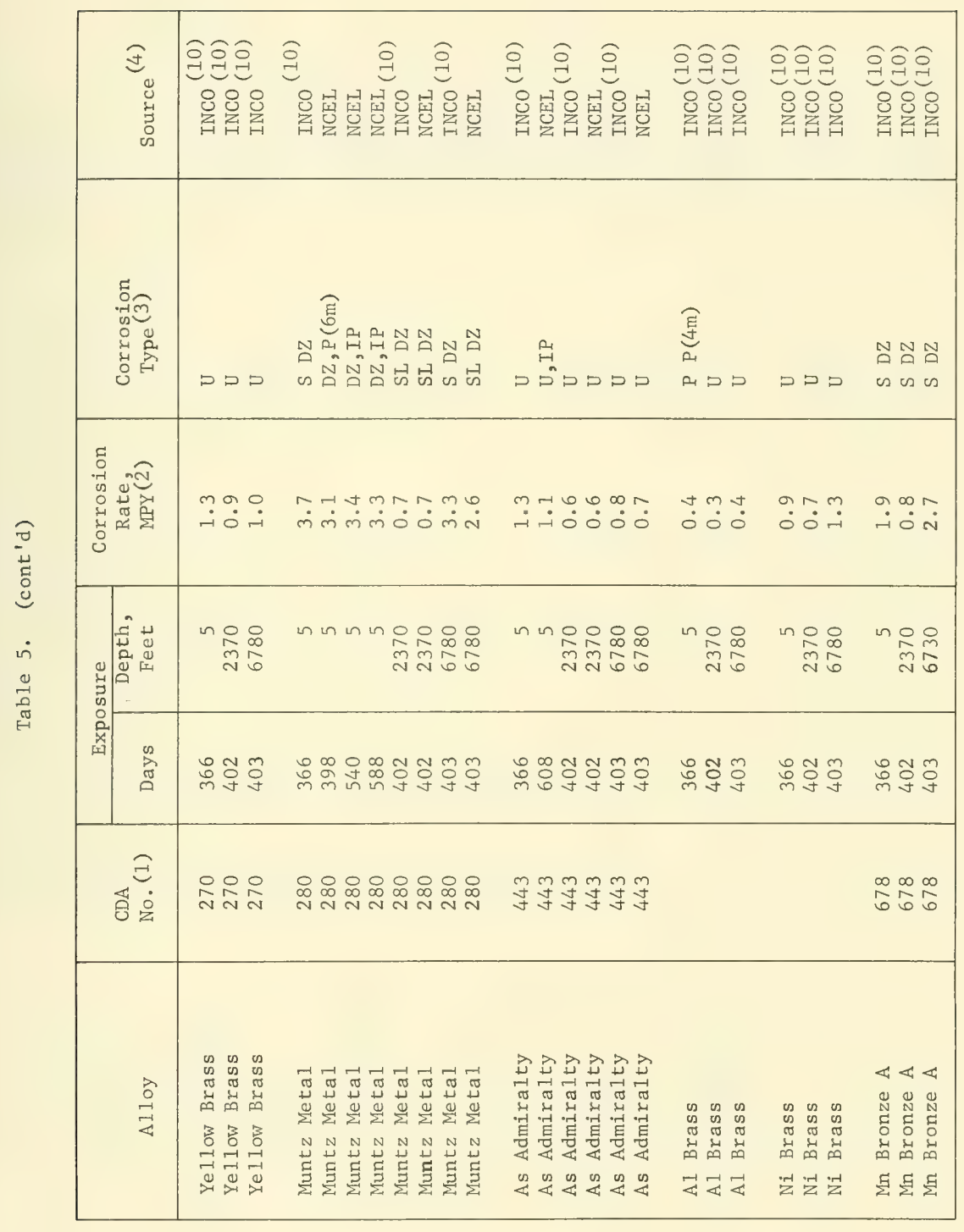




\begin{tabular}{|c|c|c|c|c|c|c|c|}
\hline 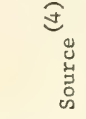 & 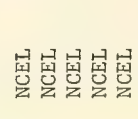 & 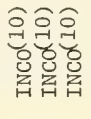 & 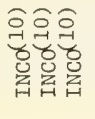 & 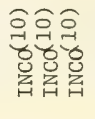 & 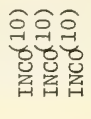 & 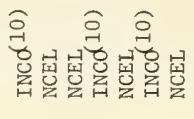 & 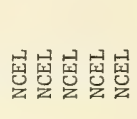 \\
\hline 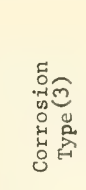 & 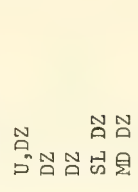 & 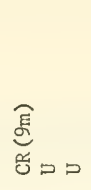 & 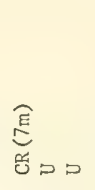 & 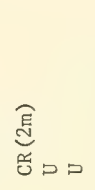 & 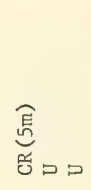 & 总 & 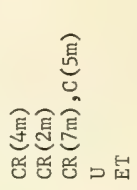 \\
\hline 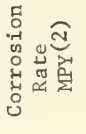 & 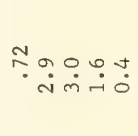 & ヘัฒ & ஜீ: & 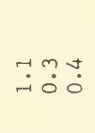 & $\dddot{m}$ in & 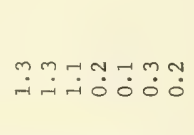 & $\ddot{0} \dot{0} \dot{0} \dot{\nabla}$ \\
\hline 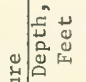 & 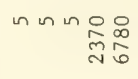 & 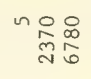 & 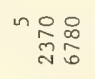 & in 0 & 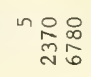 & 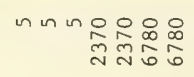 & 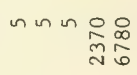 \\
\hline 福 离 & 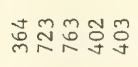 & 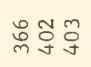 & 雚芋 & 惮号 & 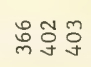 & 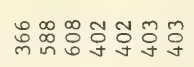 & 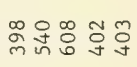 \\
\hline き & 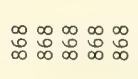 & 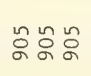 & 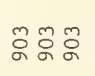 & สี สู สู & & 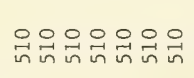 & 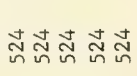 \\
\hline 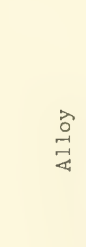 & 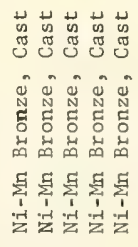 & 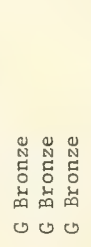 & 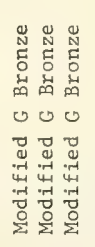 & 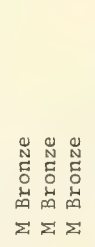 & 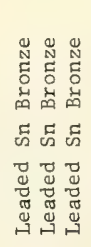 & 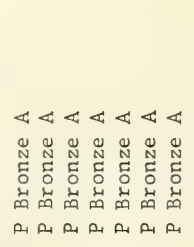 & 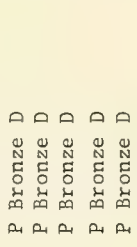 \\
\hline
\end{tabular}




\begin{tabular}{|c|c|c|c|c|c|c|c|c|}
\hline 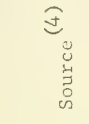 & 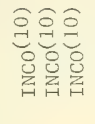 & 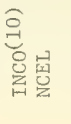 & 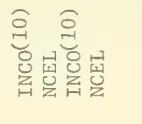 & 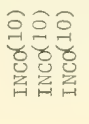 & 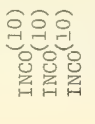 & 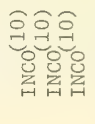 & 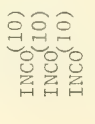 & 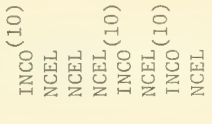 \\
\hline 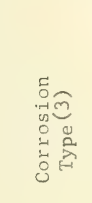 & טD的 & 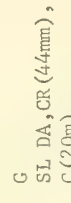 & 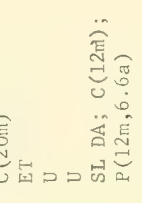 & 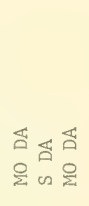 & 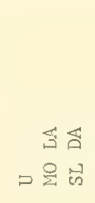 & 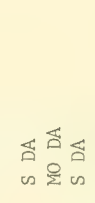 & $\begin{array}{r}8 \\
0=\frac{8}{2}\end{array}$ & 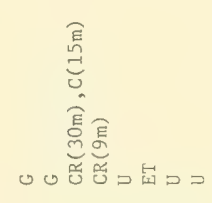 \\
\hline 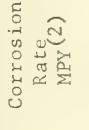 & 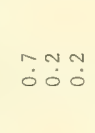 & $\begin{array}{l}00 \% \\
0: 0\end{array}$ & N N & भ̊̈ं & 풍 & 官 & تュ & 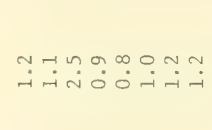 \\
\hline 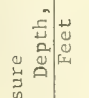 & 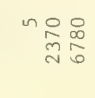 & $\operatorname{nn}$ & 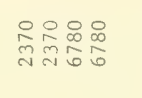 & 이요 & 们용 & 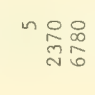 & 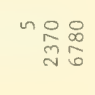 & 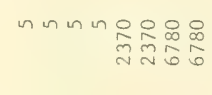 \\
\hline 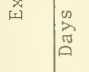 & 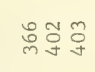 & 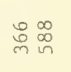 & 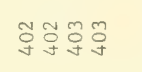 & 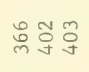 & 윰웜 & 号苧令 & 趿产号 & 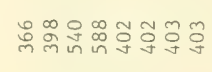 \\
\hline$\widehat{\Xi}$ & 융융 & 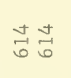 & 吉高吉寺 & 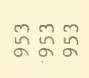 & 志芯芯 & & กู่ & 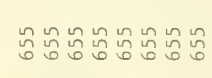 \\
\hline 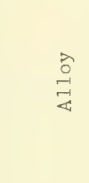 & 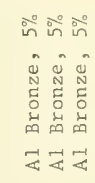 & 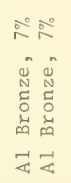 & 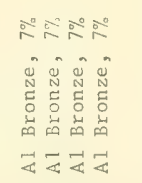 & 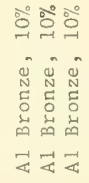 & 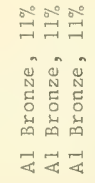 & 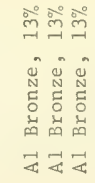 & 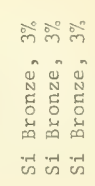 & 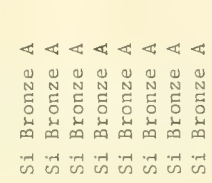 \\
\hline
\end{tabular}




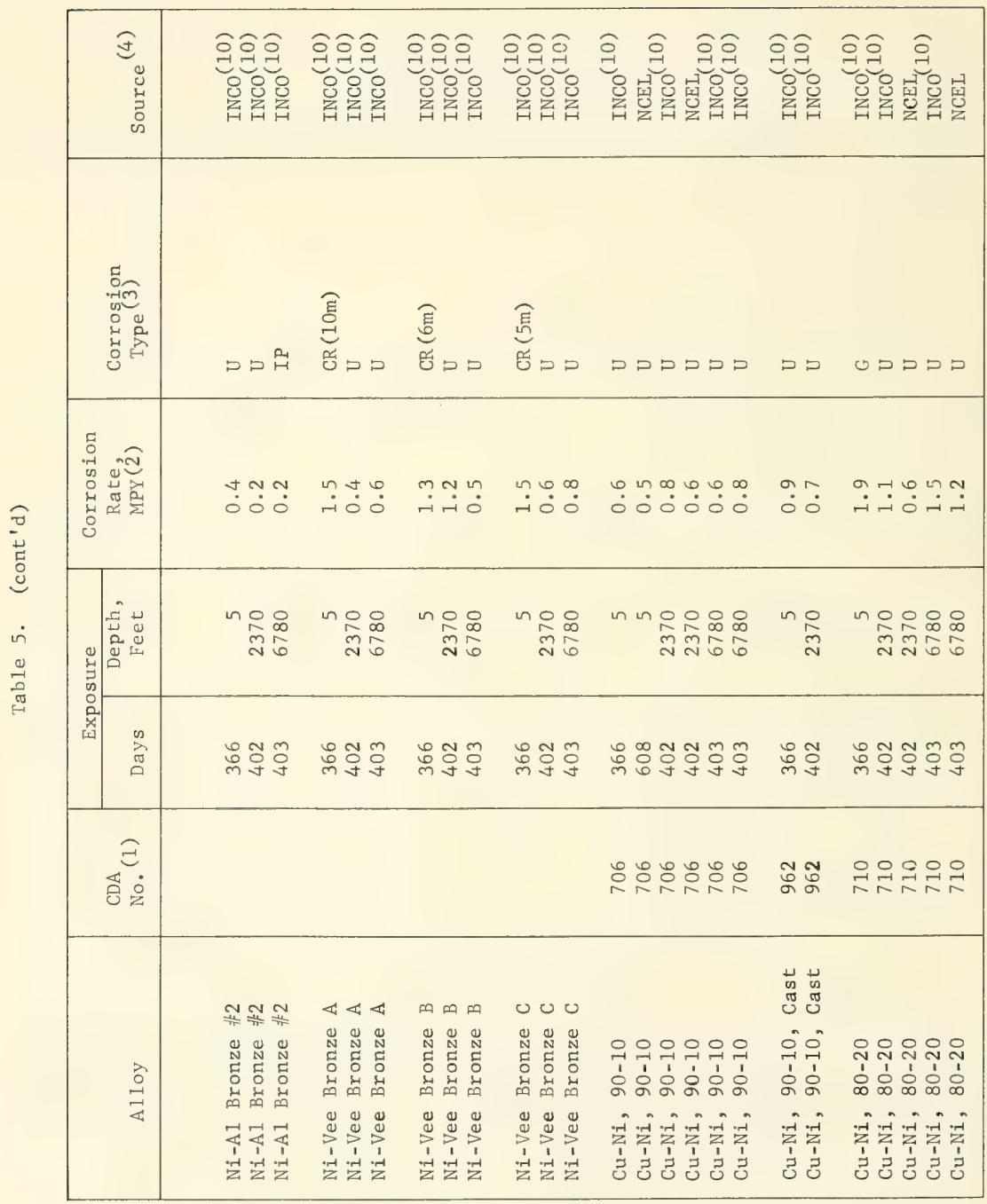




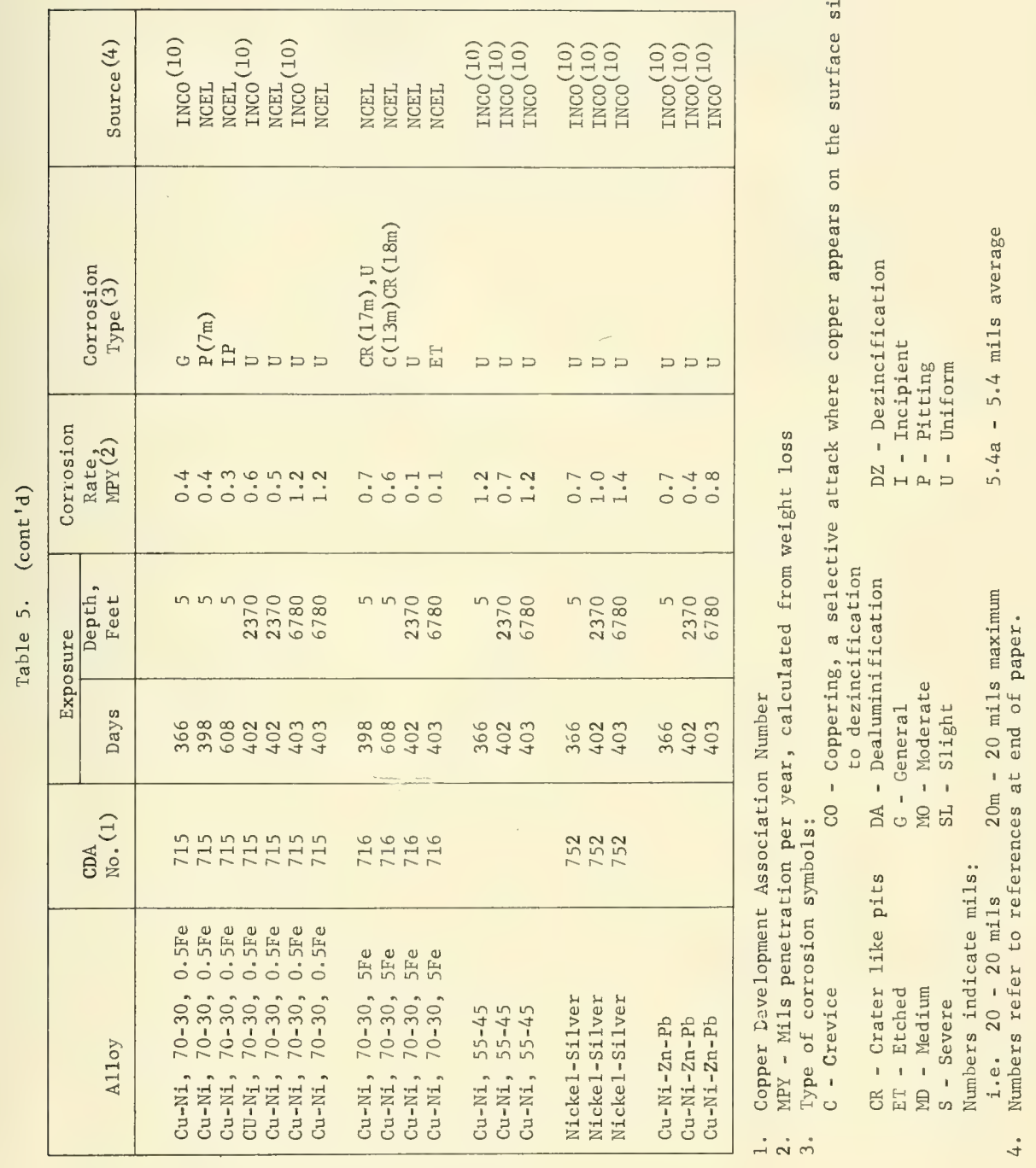




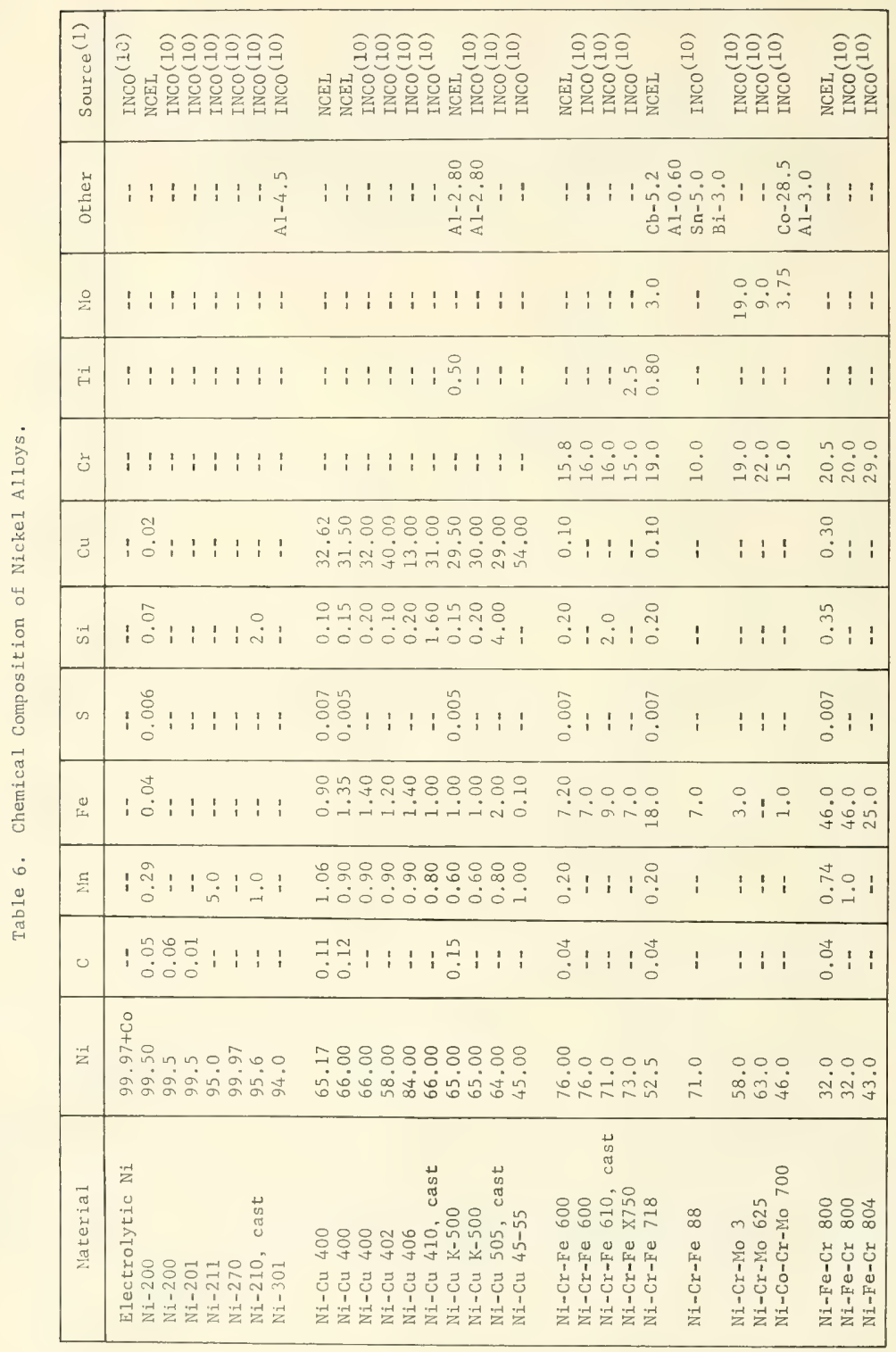




\begin{tabular}{|c|c|c|c|}
\hline 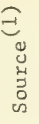 & 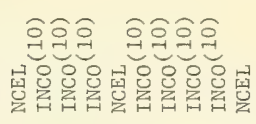 & 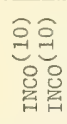 & 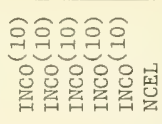 \\
\hline$\frac{\ddot{d}}{\stackrel{\Delta}{\Delta}}$ & \multicolumn{3}{|c|}{ 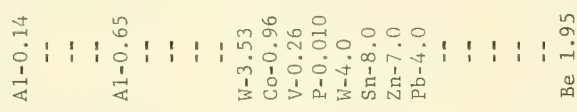 } \\
\hline$\frac{0}{2}$ & 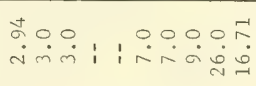 & $\stackrel{0}{0}:$ & $\begin{array}{llll}i & 1 & 0 & 0 \\
0 & i & 1\end{array}$ \\
\hline$\stackrel{-1}{H}$ & 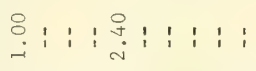 & $i:$ & 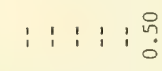 \\
\hline$\ddot{~}$ & 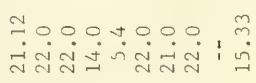 & $\stackrel{0}{\sim}:$ & 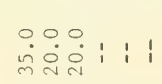 \\
\hline త & 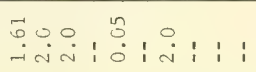 & i : & $i: 1: \dot{m} 1$ \\
\hline$\vec{\omega}$ & 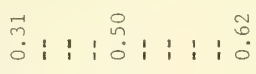 & i i & $\begin{array}{llllll}i & 1 & 1 & 1 & 0 & 1\end{array}$ \\
\hline n & 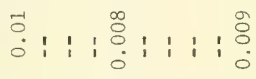 & i : & $\begin{array}{llllll}1 & 1 & 1 & 1 & 1 & 1\end{array}$ \\
\hline$\underset{\text { 幽 }}{0}$ & 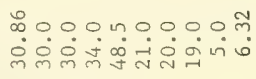 & $\stackrel{0}{\text { i : }}$ & $\begin{array}{l:llll}1 & 1 & 0 & 0 & 1\end{array}$ \\
\hline 플 & $\begin{array}{c}\infty \\
\infty \\
0\end{array}$ & $i$ & $1: 1: 1 \quad 1 \quad 11$ \\
\hline 0 & 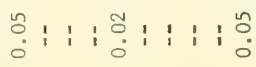 & i i & $\begin{array}{llllll}1 & 1 & 1 & 1 & 1 & 1\end{array}$ \\
\hline$\ddot{z}$ & 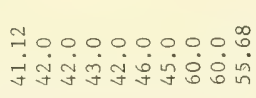 & $\stackrel{0}{0} 0$ & 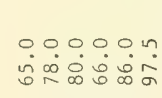 \\
\hline 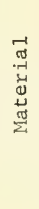 & 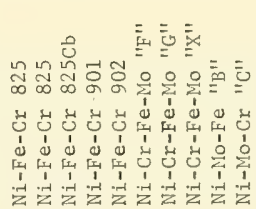 & 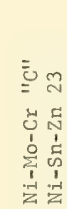 & 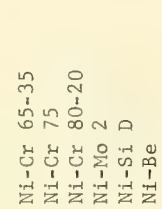 \\
\hline
\end{tabular}




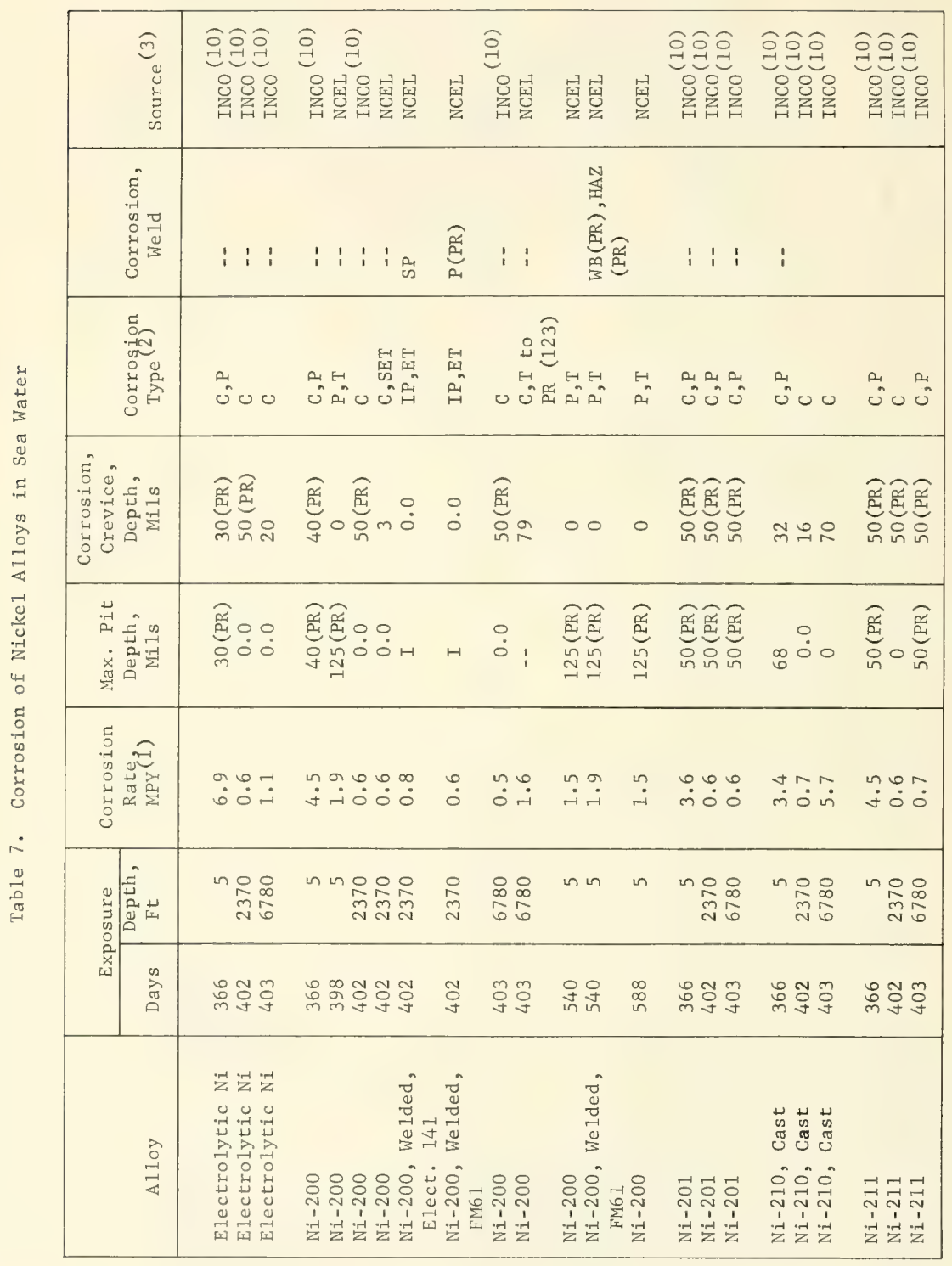




\begin{tabular}{|c|c|}
\hline 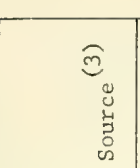 & 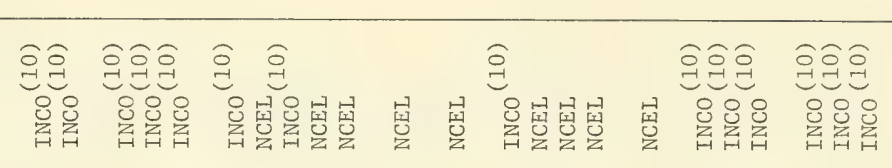 \\
\hline 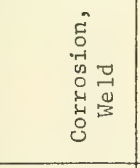 & 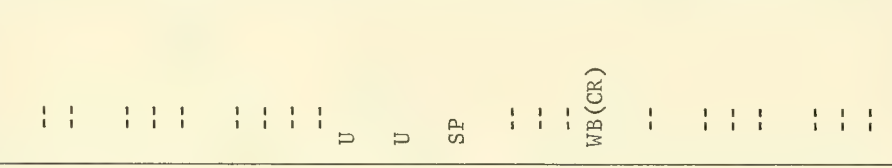 \\
\hline 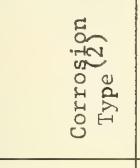 & 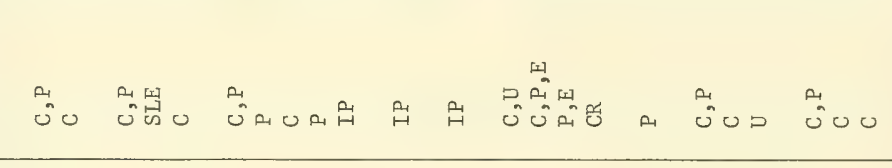 \\
\hline 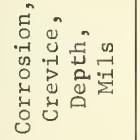 & 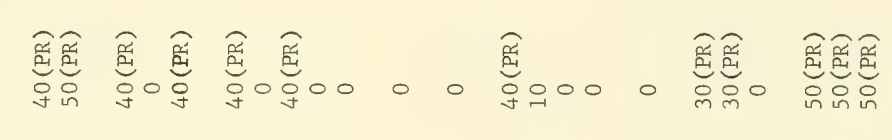 \\
\hline 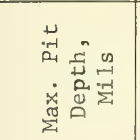 & 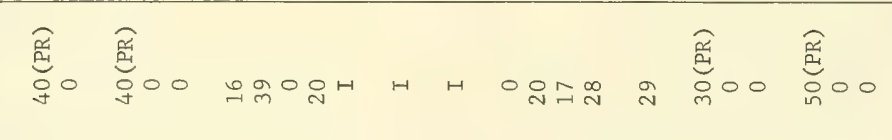 \\
\hline 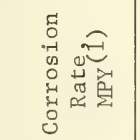 & 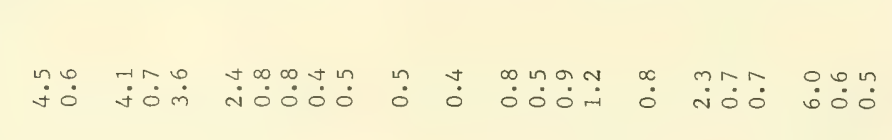 \\
\hline 這耪 & 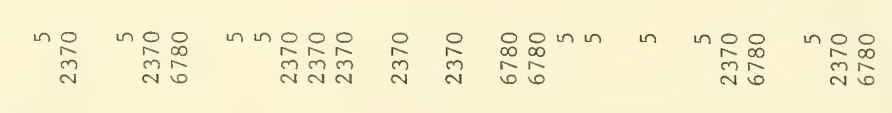 \\
\hline 辤曼 & 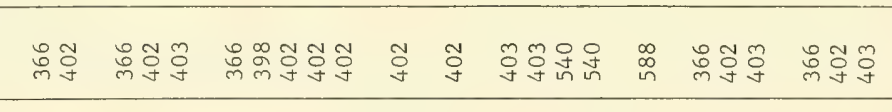 \\
\hline$\stackrel{\grave{a}}{\vec{a}}$ & 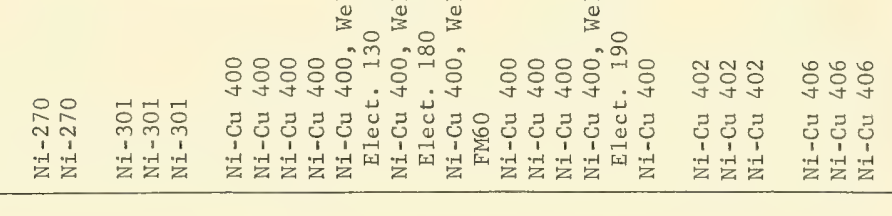 \\
\hline
\end{tabular}




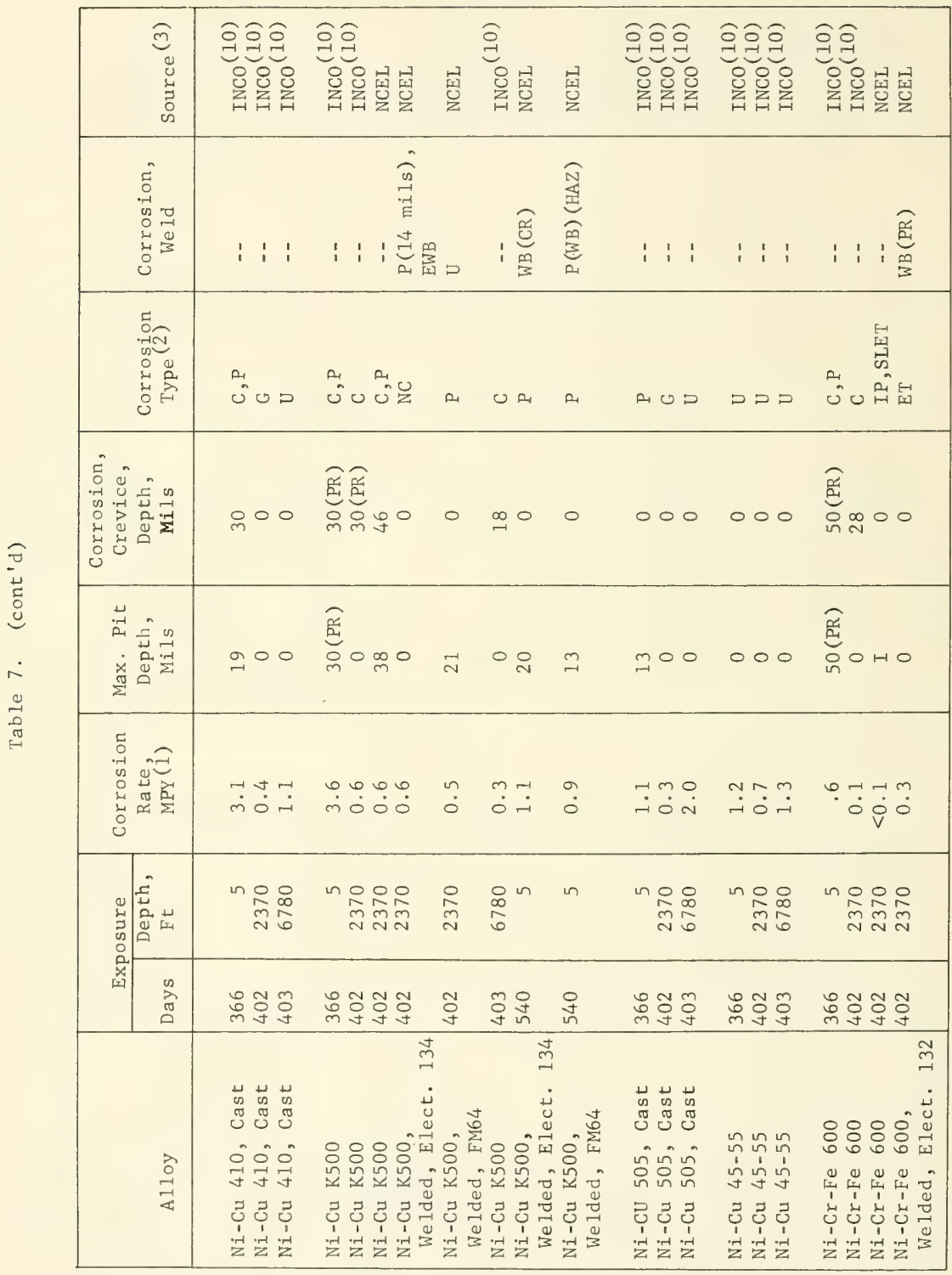




\begin{tabular}{|c|c|}
\hline 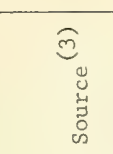 & 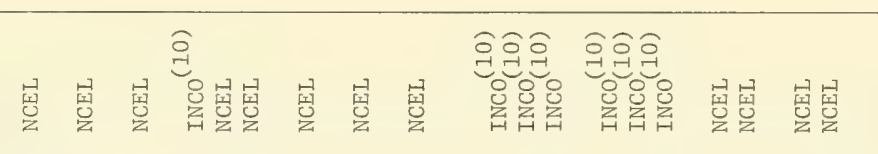 \\
\hline 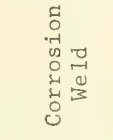 & 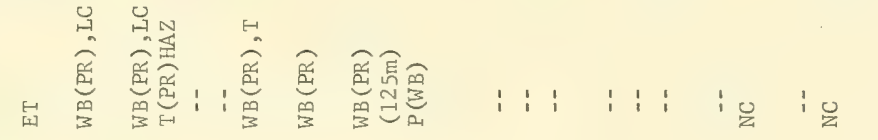 \\
\hline 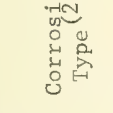 & 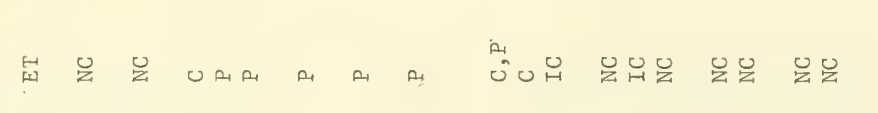 \\
\hline 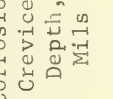 & 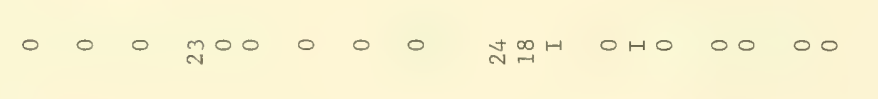 \\
\hline $\begin{array}{ll}0 \\
0\end{array}$ & 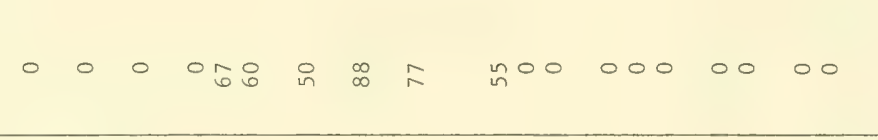 \\
\hline 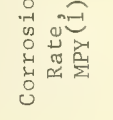 & 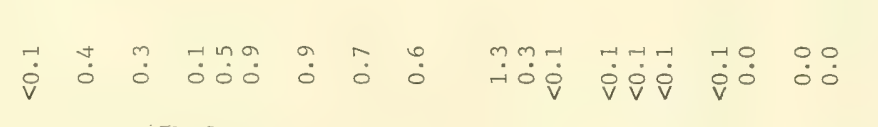 \\
\hline 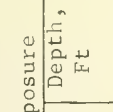 & 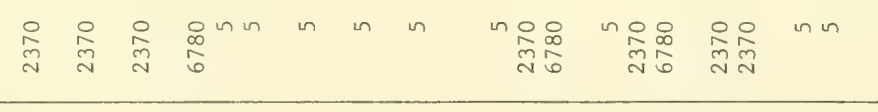 \\
\hline 番罯 & 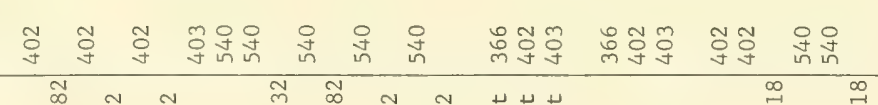 \\
\hline 高 & 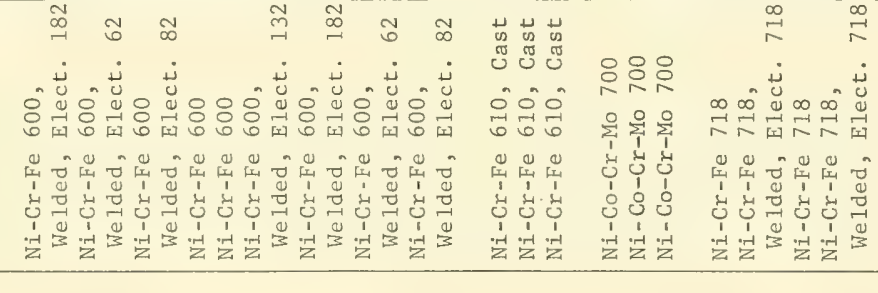 \\
\hline
\end{tabular}




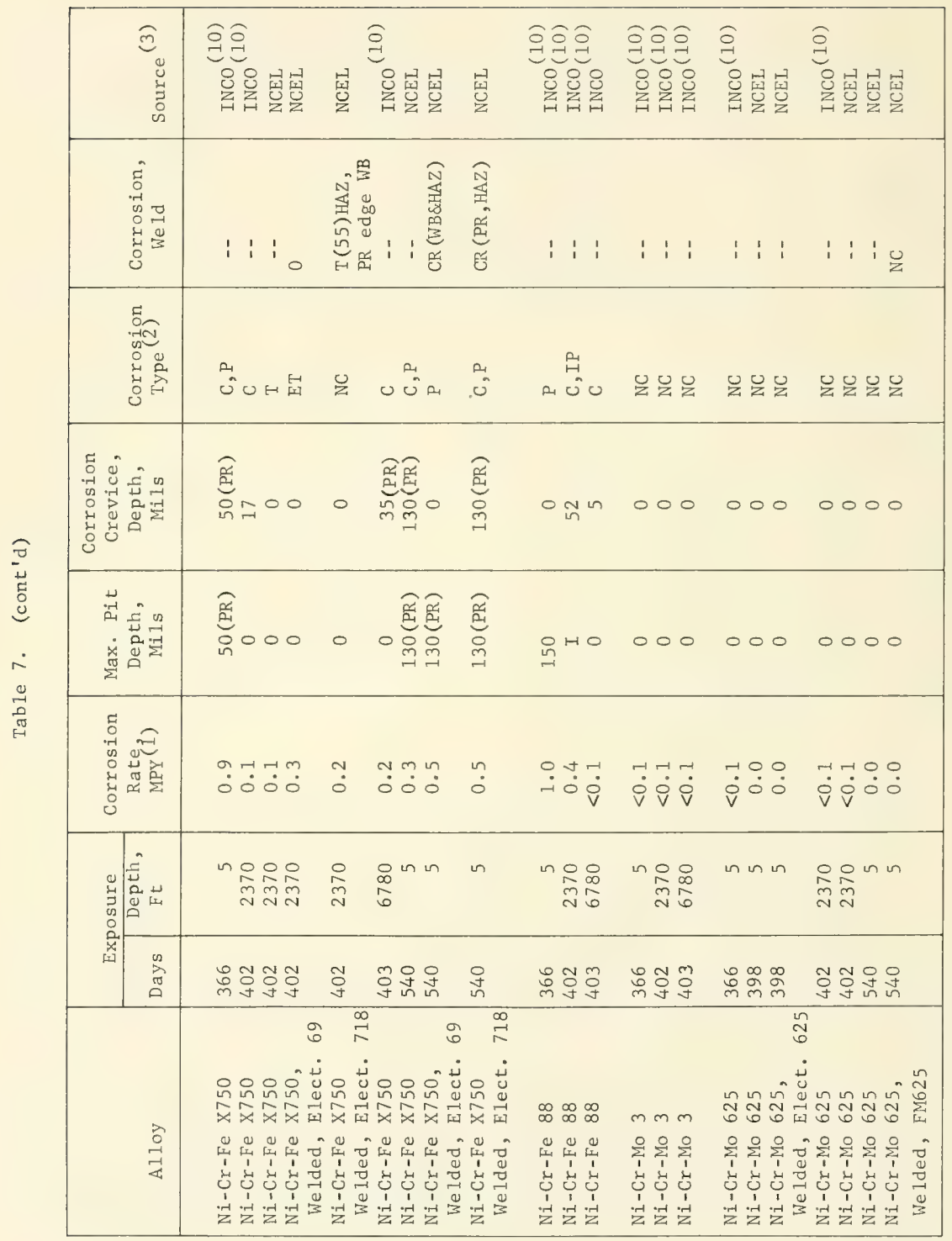




\begin{tabular}{|c|c|}
\hline 高 & 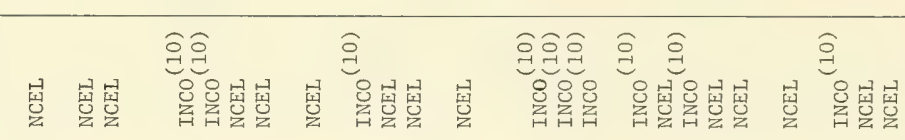 \\
\hline 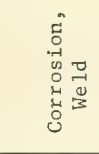 & 气 \\
\hline 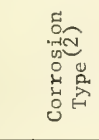 & 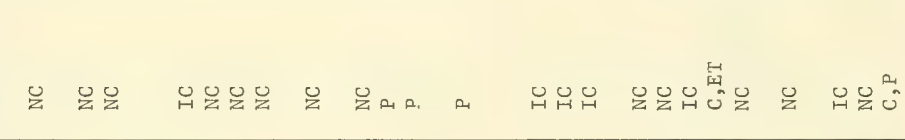 \\
\hline 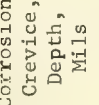 & 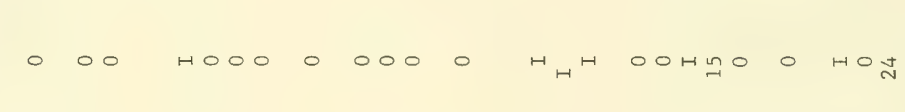 \\
\hline 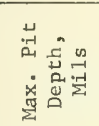 & 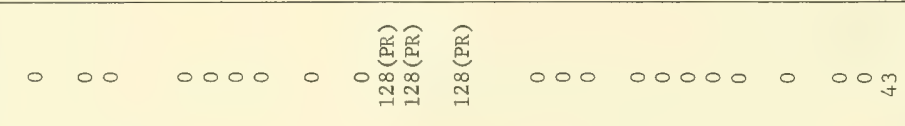 \\
\hline 资 & 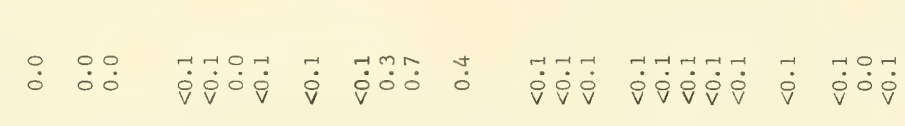 \\
\hline 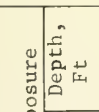 & 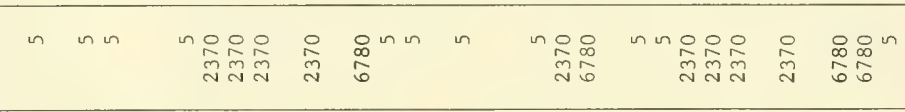 \\
\hline 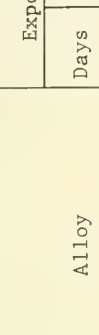 & 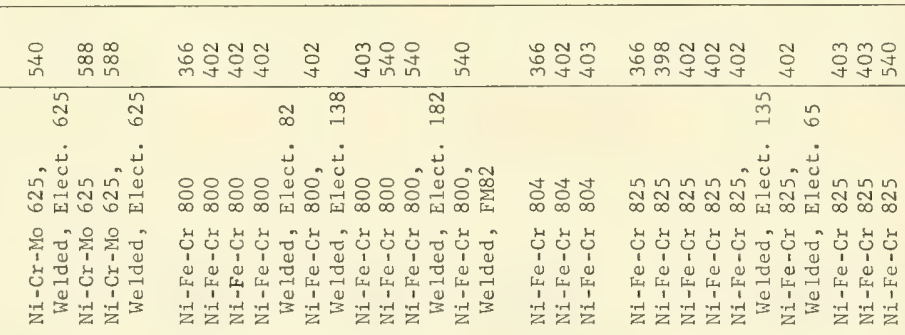 \\
\hline
\end{tabular}




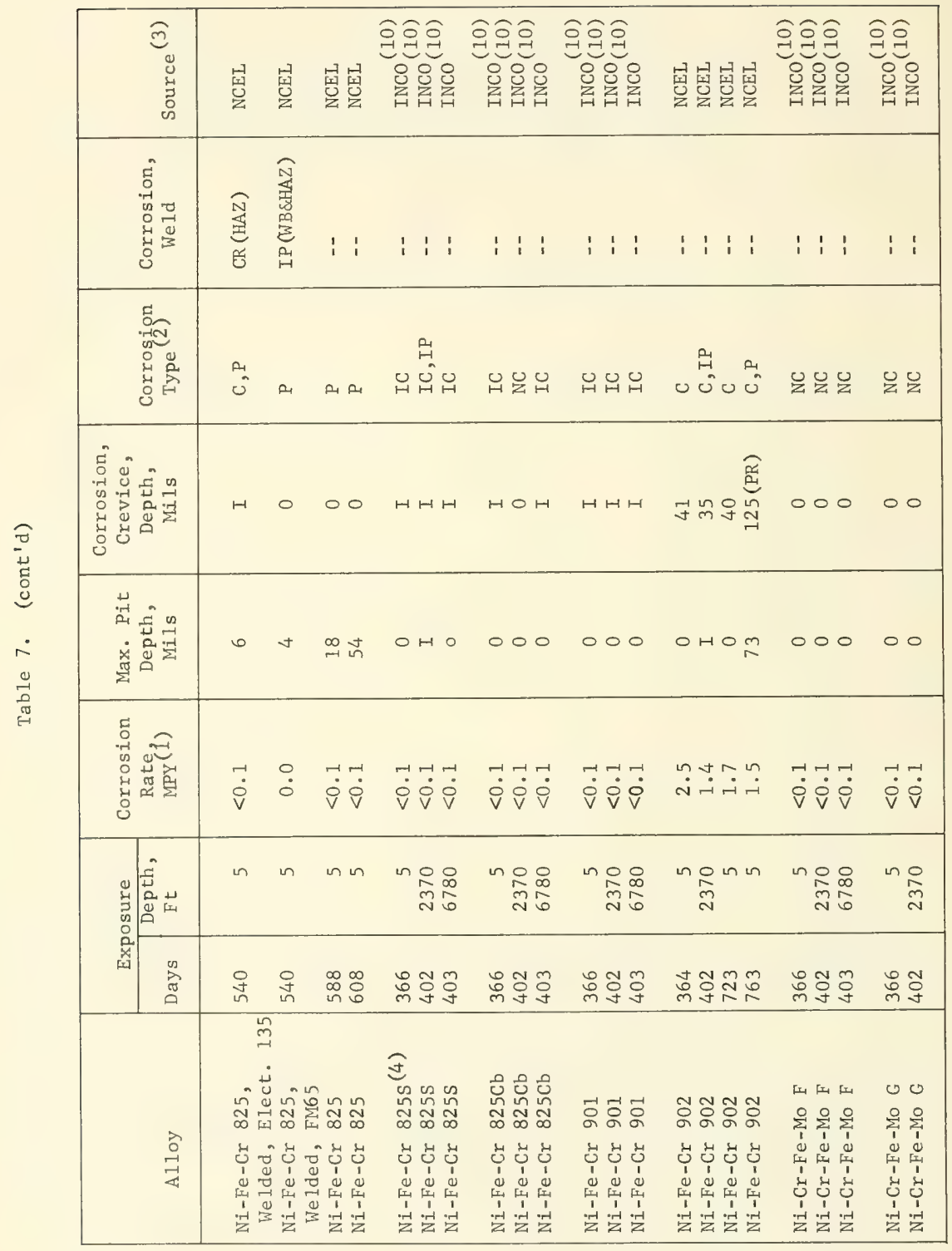




\begin{tabular}{|c|c|c|c|c|c|c|c|}
\hline 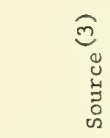 & 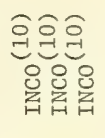 & 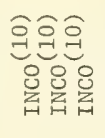 & 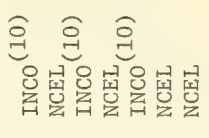 & 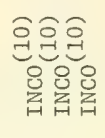 & 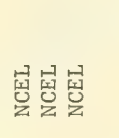 & 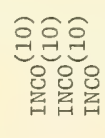 & 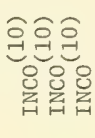 \\
\hline 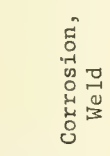 & $1 ; i$ & i i i & $\begin{array}{llllll}i & 1 & i & 1 & i & 1\end{array}$ & $1 \quad 1 \quad 1$ & $\begin{array}{lll}1 & 1 & 1\end{array}$ & $\begin{array}{l}i \\
i\end{array}$ & $i \quad i$ \\
\hline 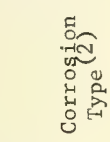 & 岂吕号 & Dט ט & 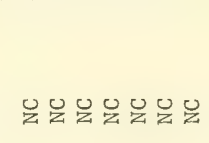 & An & 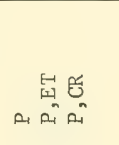 & $\begin{array}{llll}n_{1} & \\
0 & 0 & 0\end{array}$ & 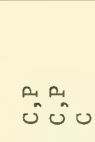 \\
\hline 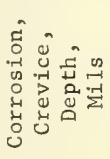 & 000 & 000 & 0000000 & $\hat{m} \stackrel{ }{m}$ & 000 & 点 & 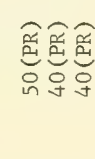 \\
\hline 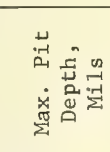 & 000 & 000 & 0000000 & $\hat{m} \circ 0$ & 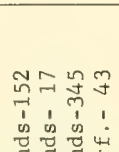 & 㣽 & 䄈虑。 \\
\hline 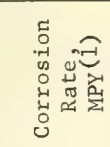 & $\overrightarrow{\dot{\theta}} \ddot{\dot{\theta}} \dot{0}$ & 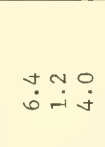 & 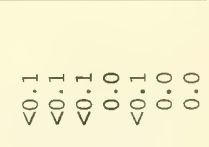 & $\begin{array}{l}n 90 \\
\dot{y} 0 \\
\dot{0}\end{array}$ & 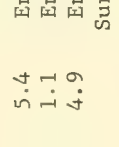 & $\ddot{\circ} \dot{0} \dot{0} \dot{0}$ & $\stackrel{-}{\sim} \dot{0} \dot{0}$ \\
\hline 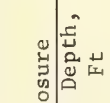 & 농요 & 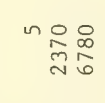 & 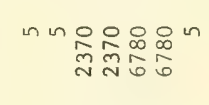 & 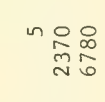 & no n & n & 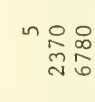 \\
\hline 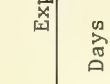 & 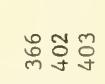 & 눙ำ & 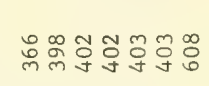 & 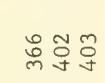 & 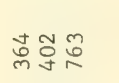 & 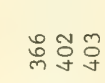 & 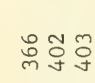 \\
\hline$\underset{\substack{2 \\
-1 \\
\frac{1}{4}}}{1}$ & 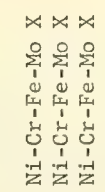 & 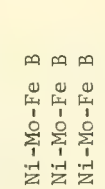 & 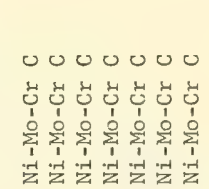 & 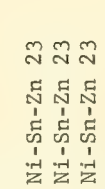 & 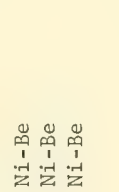 & 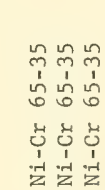 & 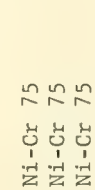 \\
\hline
\end{tabular}




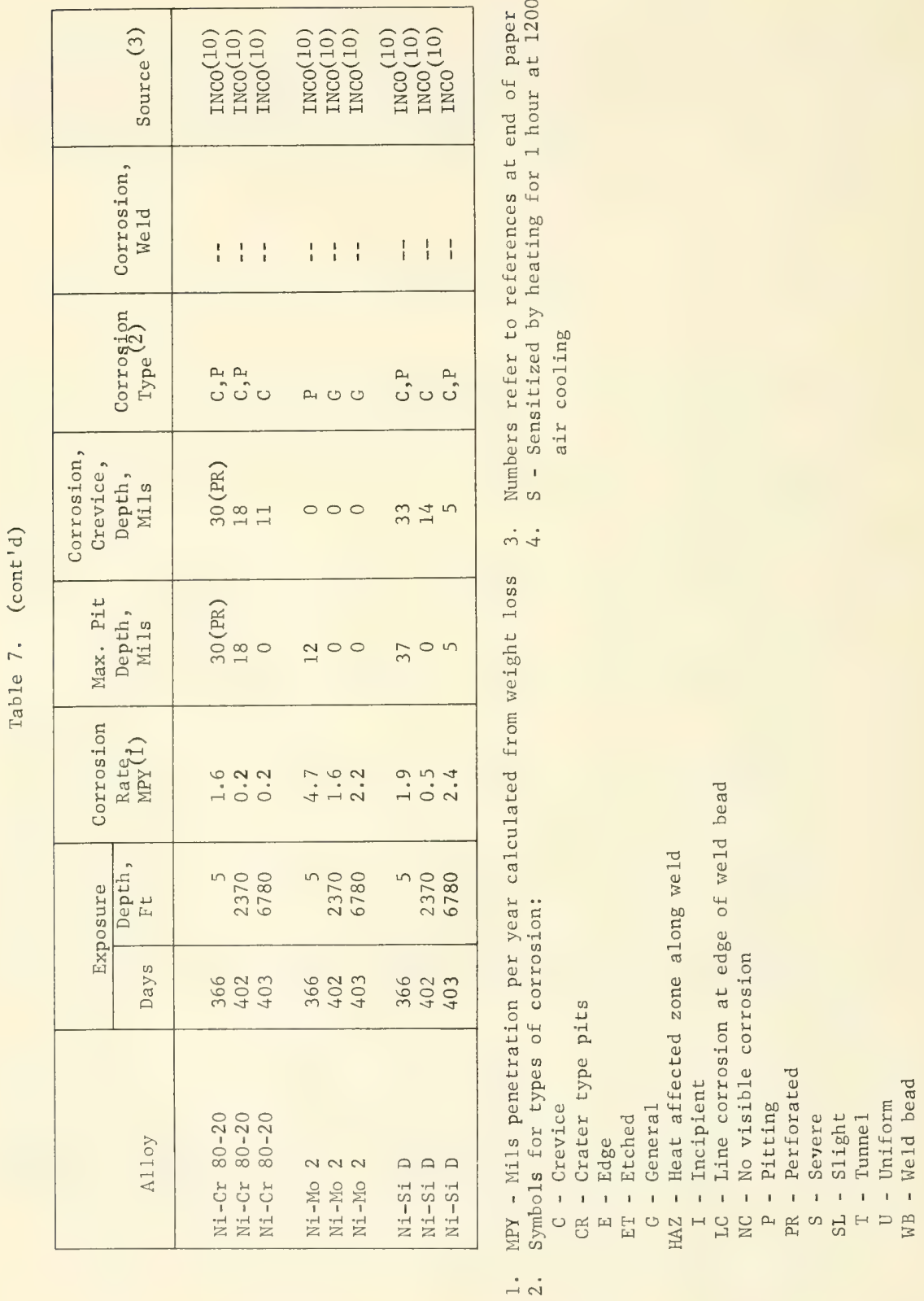




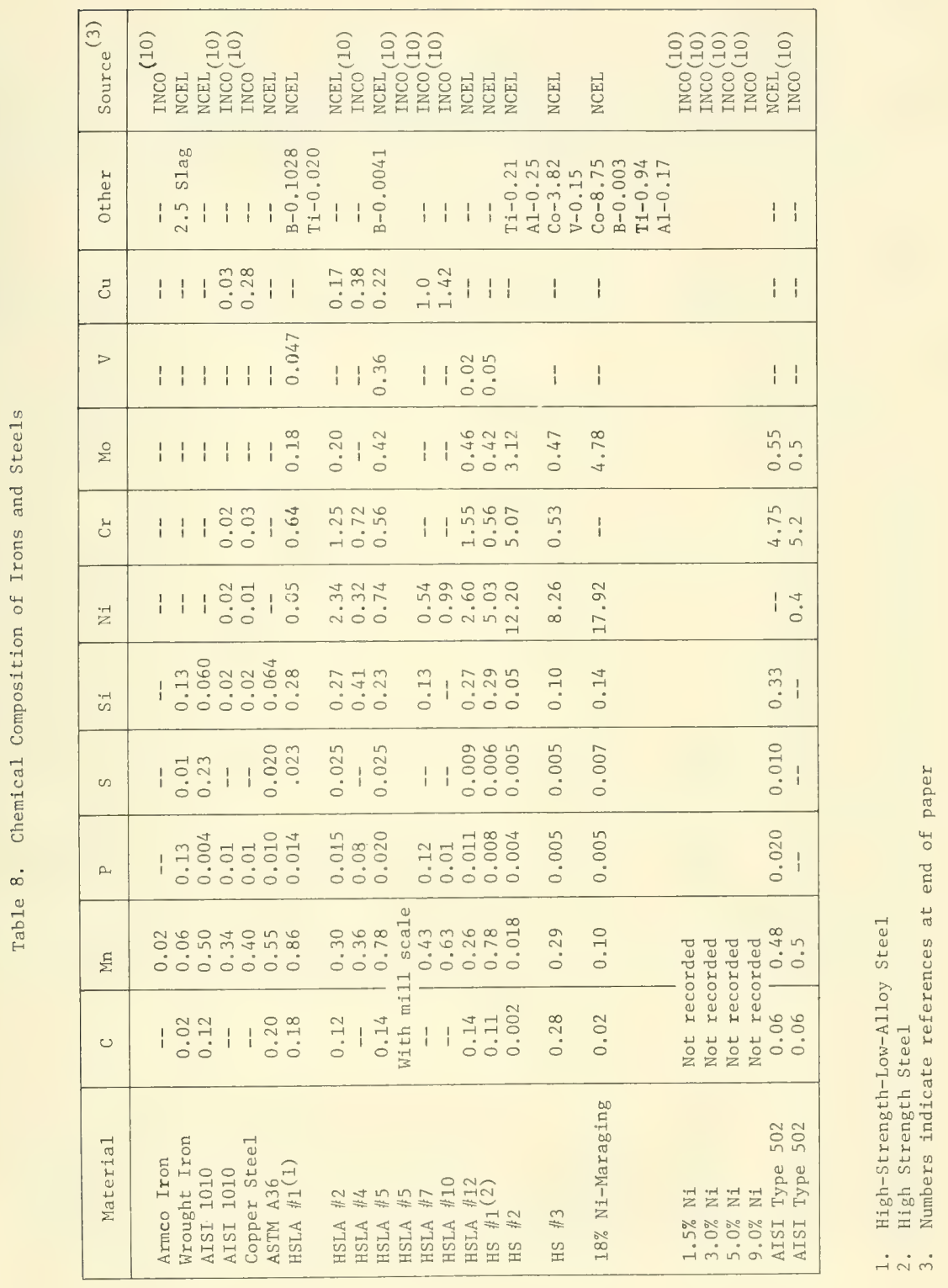




\begin{tabular}{|c|c|c|c|c|c|c|}
\hline 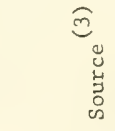 & 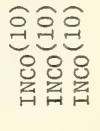 & 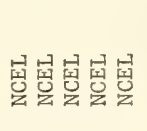 & 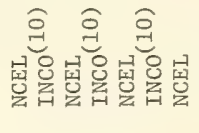 & 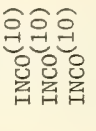 & 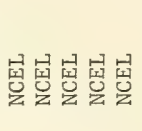 & 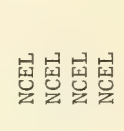 \\
\hline 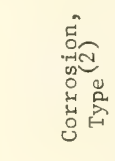 & Dט & லேロல0 & ڤ' & 0 & $\begin{array}{l}0 \\
\stackrel{H}{H} \\
\sim\end{array}$ & $\begin{array}{l}n \\
0 \\
0 \\
0\end{array}$ \\
\hline 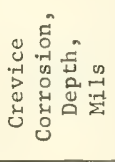 & $\begin{array}{l}1 \\
1\end{array} 1$ & $\begin{array}{lllll}1 & 1 & 1 & 1 & 1\end{array}$ & $0: 1 \quad i \quad i \sum_{n}^{n}$ & $1 \quad 1 \quad i$ & H: i & 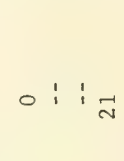 \\
\hline 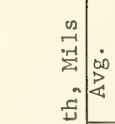 & i ' i i & i i i i i & 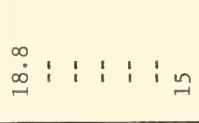 & $1 \quad 1 \quad 1$ & 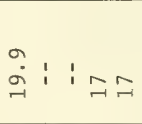 & n'i ĩ \\
\hline 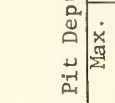 & i i i & i 1 i 11 & స & $\begin{array}{l}1 \\
1\end{array}$ & ले: ज्ञ & デ: \\
\hline 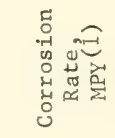 & 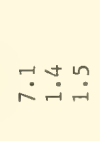 & 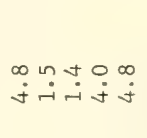 & ๓o & 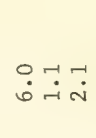 & 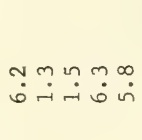 & $\begin{array}{l}\text { vo̊n } \\
\text { n் }\end{array}$ \\
\hline 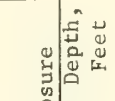 & 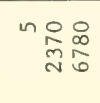 & 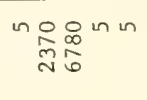 & 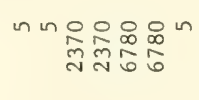 & n요요 & $\begin{array}{l}\text { no nn } \\
\text { लn } \\
\text { Nิ }\end{array}$ & $\begin{array}{l}\text { n용 } \\
\text { N } \\
\text { No }\end{array}$ \\
\hline 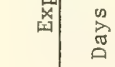 & 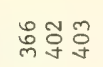 & 才。 & 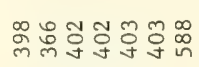 & 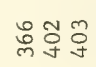 & $\begin{array}{l}\infty \\
0 \\
0\end{array}$ & 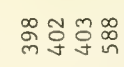 \\
\hline 总 & 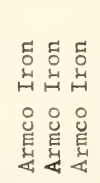 & 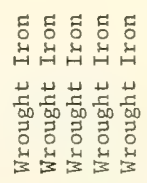 & 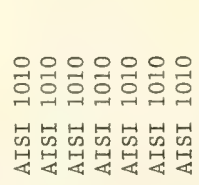 & 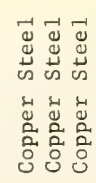 & 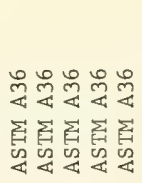 & 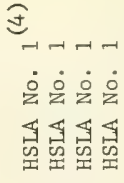 \\
\hline
\end{tabular}




\begin{tabular}{|c|c|c|c|c|c|c|}
\hline 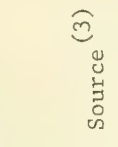 & 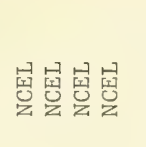 & 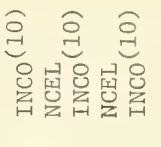 & 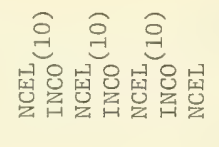 & 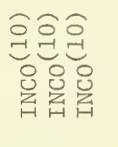 & 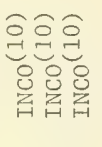 & 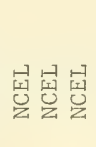 \\
\hline 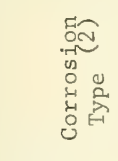 & 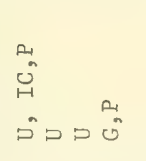 & $000=0$ & 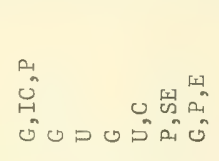 & 000 & 000 & 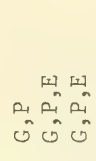 \\
\hline 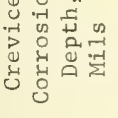 & H : : & $\begin{array}{lllll}i & 1 & 1 & i & 1\end{array}$ & $H: 1: i \stackrel{\sim}{i}: 0$ & : : : $:$ & $\begin{array}{lll}1 & : & 1\end{array}$ & 00 \\
\hline 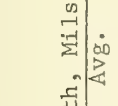 & 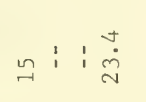 & & 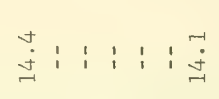 & & : $: 1:$ & 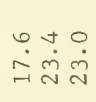 \\
\hline 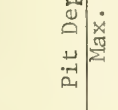 & $\therefore::_{\infty}^{\infty}$ & $\begin{array}{lllll}1 & 1 & 1 & 1 & 1\end{array}$ & $\stackrel{2}{\circ}: 1: 1: \stackrel{\circ}{m}$ & $: \quad: \quad:$ & 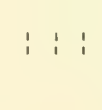 & กั่ \\
\hline 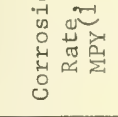 & 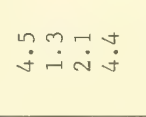 & 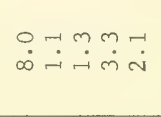 & 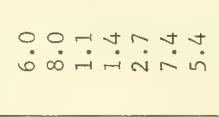 & 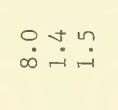 & 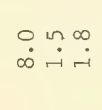 & 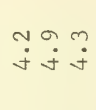 \\
\hline 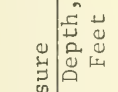 & 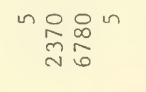 & 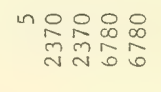 & 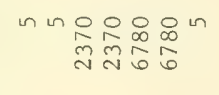 & $\begin{array}{l}\operatorname{in} \underset{0}{\infty} \\
\underset{\sim}{\infty} \frac{D}{0}\end{array}$ & 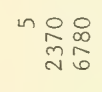 & $\ln$ in $\ln$ \\
\hline 凅 & 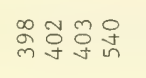 & 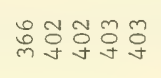 & 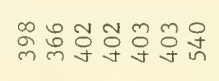 & 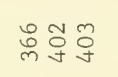 & 象旁孚 & 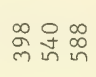 \\
\hline 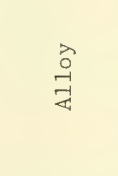 & 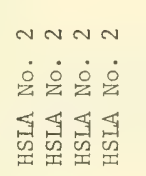 & 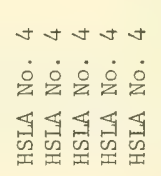 & 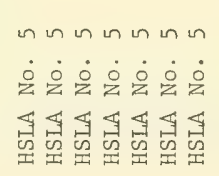 & 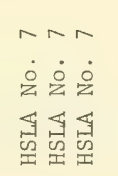 & 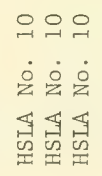 & 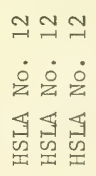 \\
\hline
\end{tabular}




\begin{tabular}{|c|c|c|c|c|c|c|c|c|}
\hline 厄ิ & 島島學 & 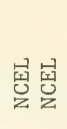 & 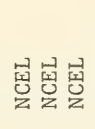 & 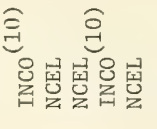 & 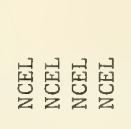 & 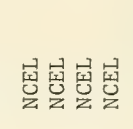 & 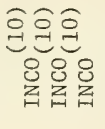 & 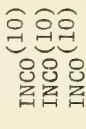 \\
\hline 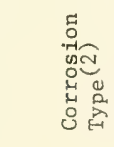 & 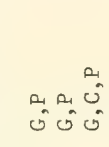 & $\begin{array}{l}n_{1}=1 \\
0^{\circ}\end{array}$ & 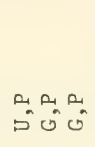 & 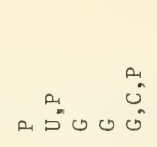 & 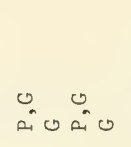 & 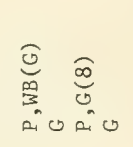 & ס כט & 000 \\
\hline 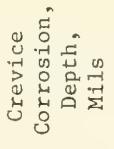 & 000 & $0=$ & 000 & 10100 & 0000 & 0000 & $\begin{array}{l}1 \\
1\end{array}$ & $11 \stackrel{0}{2}$ \\
\hline 吾 & 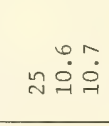 & $\begin{array}{l}a \\
\infty \\
\sim \\
\sim\end{array}$ & 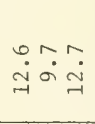 & $|\stackrel{0}{i}| 0_{\infty}^{\infty}$ & bo & rio & 1 i i & 111 \\
\hline â & $\underset{\forall}{\exists} \curvearrowleft$ & 유 ㄱ & $\stackrel{n}{\sim} \underset{\sim}{\sim} \stackrel{\infty}{-1}$ & $10010 \underset{7}{1}$ & 억엄 & $\stackrel{-1}{0} 00$ & 1 1 & 111 \\
\hline 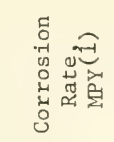 & 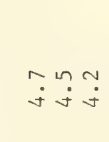 & थै भं. & $\begin{array}{l}0 \\
\dot{n} \dot{m} \dot{j}\end{array}$ & $\stackrel{\circ}{\circ}$ ஸ் & 过 थिं & $\begin{array}{l}0 \\
\dot{+} \dot{\sim} \dot{m}\end{array}$ & $\begin{array}{l}0 \text { n } \\
\infty \\
\infty\end{array}$ & $\begin{array}{l}0 m \\
\infty-1\end{array}$ \\
\hline 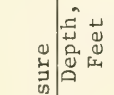 & $\ln \operatorname{nn} \ln$ & $\ln$ in & in in in & ⿰n⿴囗⿱一一) & no $\underset{N}{n}$ in in & 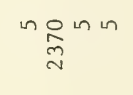 & 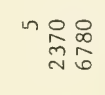 & 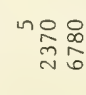 \\
\hline 齐 & $\begin{array}{l}\infty \\
\infty \\
\infty\end{array}$ & $\begin{array}{l}\infty \\
\infty \\
\infty \\
m\end{array}$ & $\begin{array}{l}\infty \\
\infty\end{array}$ & 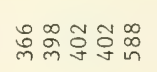 & 可设 & 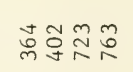 & 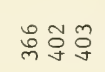 & 무요 \\
\hline$\stackrel{\hat{0}}{]}$ & 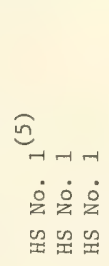 & $\begin{array}{l}N \sim \\
\dot{0}: \\
\dot{z} \\
0 \\
0\end{array}$ & 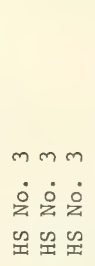 & 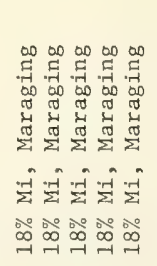 & 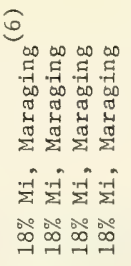 & 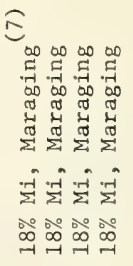 & 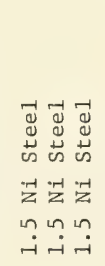 & 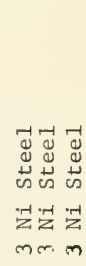 \\
\hline
\end{tabular}




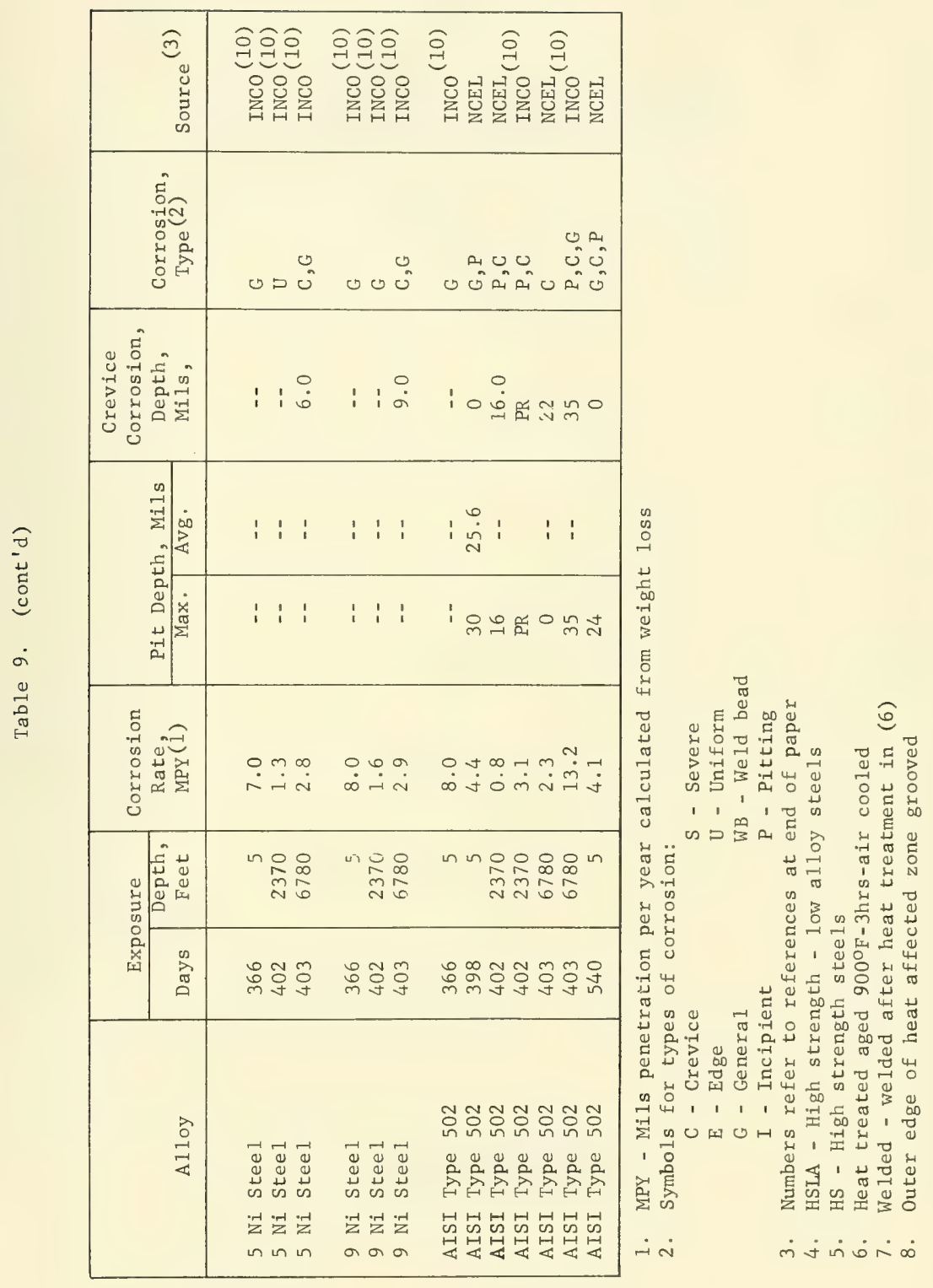




\begin{tabular}{|c|c|}
\hline 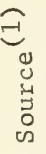 & 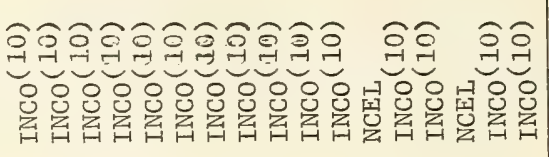 \\
\hline$\vec{u}$ & 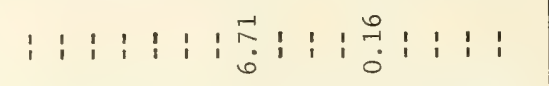 \\
\hline$\frac{0}{\Sigma}$ & $\begin{array}{llllllllllllllll}1 & 1 & 1 & 1 & 1 & 1 & 0 & 1 & 1 & 1 & 1 & 1 & 1 & 1 & 1 & 1\end{array}$ \\
\hline نे & 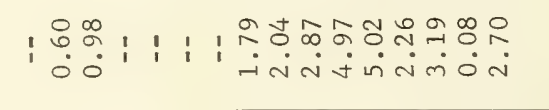 \\
\hline${ }^{-1-1}$ & 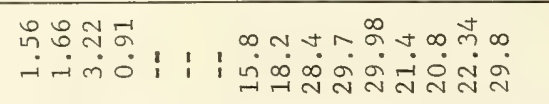 \\
\hline in & 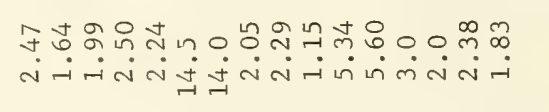 \\
\hline$\stackrel{\mathcal{L}}{\Sigma}$ & 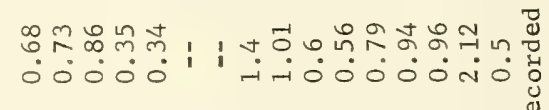 \\
\hline 0 & 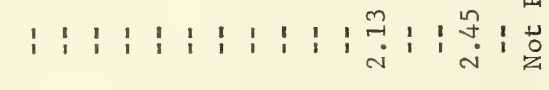 \\
\hline 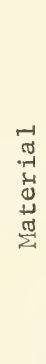 & 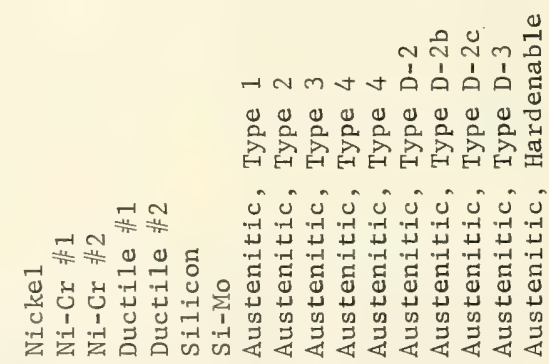 \\
\hline
\end{tabular}


Table 11. Corrosion of Cast Irons in Sea Water

\begin{tabular}{|c|c|c|c|c|c|}
\hline \multirow[b]{2}{*}{ Alloy } & \multicolumn{2}{|c|}{ Exposure } & \multirow{2}{*}{$\begin{array}{c}\text { Corrosion } \\
\text { Rate } \\
\text { MPY (1) }\end{array}$} & \multirow[b]{2}{*}{$\begin{array}{c}\text { Corrosion } \\
\text { Type (2) }\end{array}$} & \multirow[b]{2}{*}{ Source (3) } \\
\hline & Days & $\begin{array}{l}\text { Depth, } \\
\text { Ft }\end{array}$ & & & \\
\hline Gray & 366 & 5 & 2.6 & G & INCO $(10)$ \\
\hline Gray & 402 & 2370 & 1.7 & $\mathrm{U}$ & INCO $(10)$ \\
\hline Gray & 403 & 6780 & 1.8 & $\mathrm{U}$ & INCO $(10)$ \\
\hline Nickel & 366 & 5 & 7.6 & G. & INCo $(10)$ \\
\hline Nickel & 402 & 2370 & 1.5 & U & INCO (10) \\
\hline Nickel & 403 & 6780 & 2.9 & $\mathrm{l}$ & INCO \\
\hline $\mathrm{Ni}-\mathrm{Cr} \# 1$ & 366 & 5 & 5.2 & $\mathrm{U}$ & INCO $(10)$ \\
\hline $\mathrm{Ni}-\mathrm{Cr}$ & 402 & 2370 & 1.8 & $\mathrm{U}$ & INCO (10) \\
\hline $\mathrm{Ni}-\mathrm{Cr} \# 1$ & 403 & 6780 & 1.7 & U & INCO $(10)$ \\
\hline $\mathrm{Ni}-\mathrm{Cr} \| 2$ & 366 & 5 & 4.9 & G & INCO $(10)$ \\
\hline $\mathrm{Ni}-\mathrm{Cr} \# 2$ & 402 & 2370 & 1.8 & U & INCO (10) \\
\hline $\mathrm{Ni}-\mathrm{Cr} \| 2$ & 403 & 6780 & 1.8 & U & INco $(10)$ \\
\hline Ductile \#1 & 366 & 5 & 6.2 & $\mathrm{CR}(24 \mathrm{~m})$ & INCO $(10)$ \\
\hline Ductile $\| 1$ & 402 & 2370 & 1.9 & $\mathrm{U}$ & INCO (10) \\
\hline Ductile 1 & 403 & 6780 & 3.4 & G & INCO $(10)$ \\
\hline Duct11e $\# 2$ & 366 & 5 & 7.1 & G & INCO (10) \\
\hline Ductile " 2 & 402 & 2370 & 1.8 & u & INCO $(10)$ \\
\hline Ductile $\# 2$ & 403 & 6780 & 2.9 & G & INCO $(10)$ \\
\hline Sillcon & 366 & 5 & -0.1 & ET & INCO (10) \\
\hline Silicon & 402 & 2370 & $<0.1$ & NC & INCO (10) \\
\hline Silicon & 403 & 6780 & $<0.1$ & $\mathrm{NC}$ & INCO $(10)$ \\
\hline $\mathrm{Si}-\mathrm{Mo}$ & 366 & 5 & $<0.1$ & ET & INCO (10) \\
\hline Si-Yo & 402 & 2370 & $<0.1$ & $\mathrm{NC}$ & INCO (10) \\
\hline Si-Mo & 403 & 6780 & $<0.1$ & NC & INCO $(10)$ \\
\hline Austenitic, Type I & 366 & 5 & 2.7 & U & INCO (10) \\
\hline Austenitic, Type 1 & 402 & 2370 & 1.5 & $\mathrm{U}$ & INCO $(10)$ \\
\hline Austenitic, Type 1 & 403 & 6780 & 1.0 & $\mathrm{U}$ & INCO $(10)$ \\
\hline Austenitic, Type 2 & 366 & 5 & 2.9 & U & INCO (10) \\
\hline Austenitic, Type 2 & 402 & 2370 & 1.1 & $\mathrm{U}$ & INCO $(10)$ \\
\hline Austenitic, Type 2 & 403 & 6780 & 2.2 & $\mathrm{U}$ & INCO $(10)$ \\
\hline
\end{tabular}


Table 11. (cont'd)

\begin{tabular}{|c|c|c|c|c|c|}
\hline \multirow[b]{2}{*}{ Alloy } & \multicolumn{2}{|c|}{ Exposure } & \multirow{2}{*}{$\begin{array}{c}\text { Corrosion } \\
\text { Rate, } \\
\text { MPY (i) }\end{array}$} & \multirow[b]{2}{*}{$\begin{array}{l}\text { Corrosion } \\
\text { Type (2) }\end{array}$} & \multirow[b]{2}{*}{ Source ${ }^{(3)}$} \\
\hline & Days & $\begin{array}{l}\text { Depth, } \\
\text { Ft }\end{array}$ & & & \\
\hline Austenitic, Type 3 & 366 & 5 & 2.8 & $\mathrm{U}$ & $\operatorname{INCO}(10)$ \\
\hline Austenitic, Type 3 & 402 & 2370 & 0.6 & $\mathrm{U}$ & INCO $(10)$ \\
\hline Austenitic, Type 3 & 403 & 6780 & 1.8 & $\mathrm{U}$ & $\operatorname{INCO}(10)$ \\
\hline Austenitic, Type 4 & 366 & 5 & 2.4 & $\mathrm{U}$ & $\operatorname{INCO}(10)$ \\
\hline Austenitic, Type 4 & 364 & 5 & 2.4 & G & NCEL \\
\hline Austenitic, Type 4 & 402 & 2370 & 0.8 & $\mathrm{U}$ & $\operatorname{INCO}^{(10)}$ \\
\hline Austenitic, Type 4 & 402 & 2370 & 0.9 & G & NCEL \\
\hline Austenitic, Type 4 & 403 & 6780 & 2.0 & $\mathrm{U}$ & $\mathrm{INCO}^{(10)}$ \\
\hline Austenitic, Type 4 & 723 & 5 & 2.0 & G & NCEL \\
\hline Austenitic, Type 4 & 763 & 5 & 2.0 & G & NCEL \\
\hline Austenitic, Type D-2 & 366 & 5 & 2.4 & G & $\operatorname{INCO}(10)$ \\
\hline Austenitic, Type D-2 & 402 & 2370 & 1.1 & $\mathrm{U}$ & INCO $(10)$ \\
\hline Austenitic, Type D-2 & 403 & 6780 & 1.2 & $\mathrm{U}$ & $\operatorname{INCO}^{(10)}$ \\
\hline Austenitic, D-2B & 366 & 5 & 2.7 & G & INCO $(10)$ \\
\hline Austenitic, D-2B & 402 & 2370 & 0.9 & U & INCO (10) \\
\hline Austenitic, D-2B & 403 & 6780 & 1.6 & U & $\operatorname{INCO}^{(10)}$ \\
\hline Austenitic, D-2C & 364 & 5 & 3.2 & G & NCEL \\
\hline Austenitic, D-2C & 402 & 2370 & 1.8 & $\mathrm{U}$ & NCEL \\
\hline Austenitic, D-2C & 723 & 5 & 3.1 & $\mathrm{U}$ & NCEL \\
\hline Austenitic, D-2C & 763 & 5 & 2.8 & $\mathrm{U}$ & NCEL \\
\hline Austenitic, D-3 & 366 & 5 & 3.2 & G & $\operatorname{INCO}(10)$ \\
\hline Austenitic, D-3 & 402 & 2370 & 0.7 & $\mathrm{U}$ & $\mathrm{INCO}_{(10)}$ \\
\hline Austenitic, D-3 & 403 & 6780 & 2.7 & G & $\mathrm{INCO}^{(10)}$ \\
\hline $\begin{array}{l}\text { Austenitic, } \\
\text { Hardenable }\end{array}$ & 366 & 5 & 2.6 & $\mathrm{U}$ & $\operatorname{INCO}^{(10)}$ \\
\hline $\begin{array}{l}\text { Austenitic, } \\
\text { Hardenable }\end{array}$ & 402 & 2370 & 1.8 & $\mathrm{U}$ & $\operatorname{INCO}^{(10)}$ \\
\hline $\begin{array}{l}\text { Austenitic, } \\
\text { Hardenable }\end{array}$ & 403 & 6780 & 1.1 & $\mathrm{U}$ & $\operatorname{INCO}^{(10)}$ \\
\hline
\end{tabular}

1. MPY - Mils penetration per year calculated from weight loss

2. Symbols for types of corrosion:

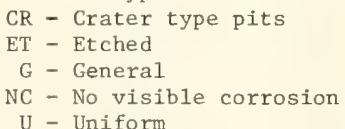

3. Numbers refer to references at end of paper 


\begin{tabular}{|c|c|c|}
\hline 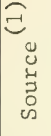 & 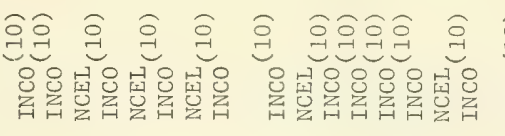 & 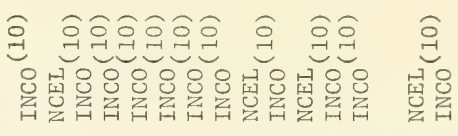 \\
\hline$\stackrel{\substack{0\\
}}{\Delta}$ & $\begin{array}{llllllll}1 & 1 & 1 & 1 & 1 & 1 & 1 & 1\end{array}$ & 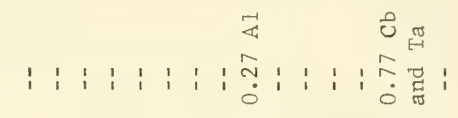 \\
\hline ב & 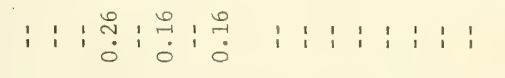 & 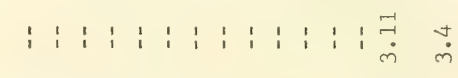 \\
\hline$\stackrel{2}{2}$ & 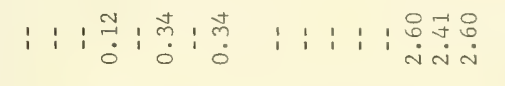 & 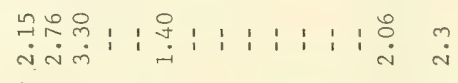 \\
\hline பै & 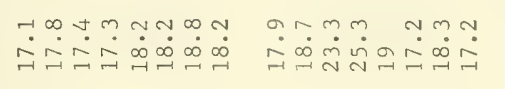 & 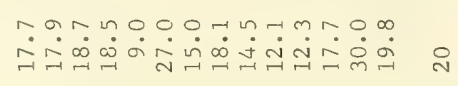 \\
\hline$\ddot{z}$ & 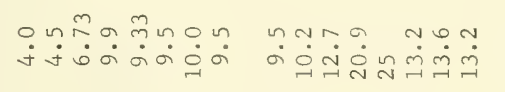 & 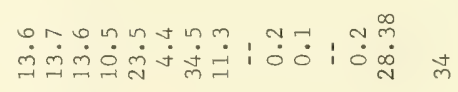 \\
\hline ت્1 & 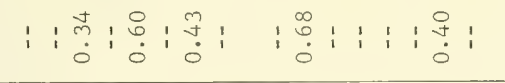 & 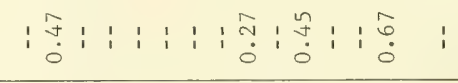 \\
\hline us & 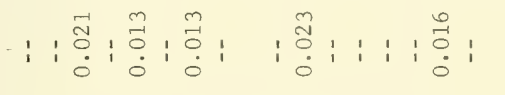 & 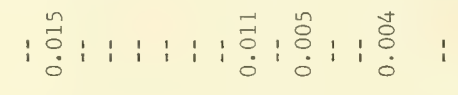 \\
\hline A & 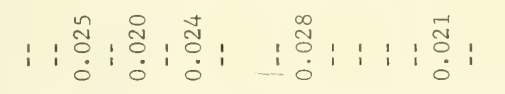 & 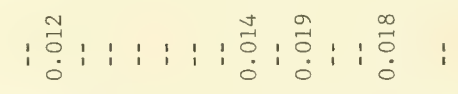 \\
\hline 돌 & 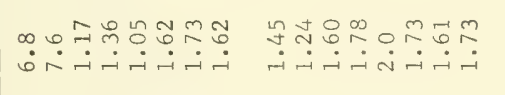 & 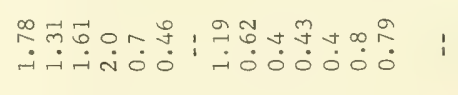 \\
\hline 0 & 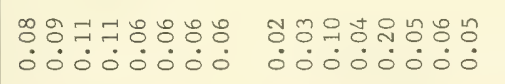 & 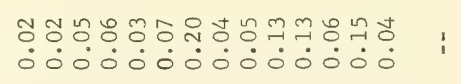 \\
\hline 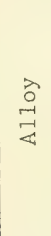 & 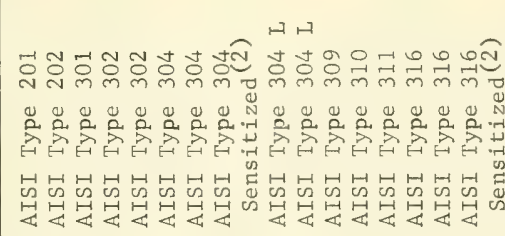 & 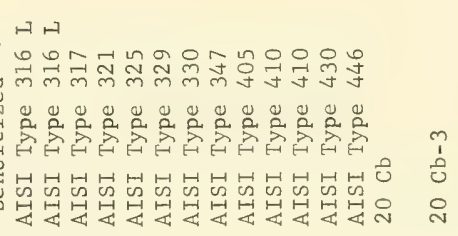 \\
\hline
\end{tabular}




\begin{tabular}{|c|c|}
\hline 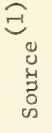 & 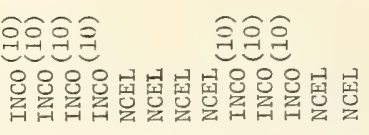 \\
\hline 苛 & 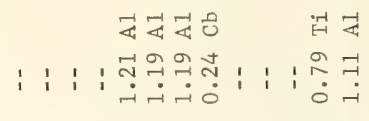 \\
\hline $\overrightarrow{0}$ & 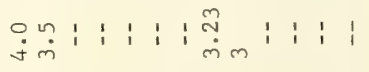 \\
\hline 을 & 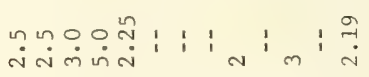 \\
\hline نे & 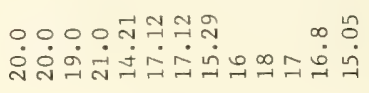 \\
\hline 资 & 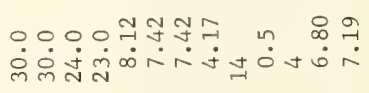 \\
\hline$\ddot{n s}$ & i : : \\
\hline w & 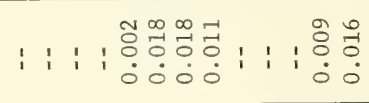 \\
\hline D. & 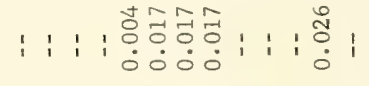 \\
\hline 돌 & i i : i \\
\hline 0 & 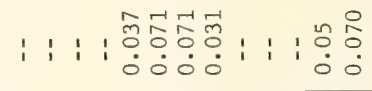 \\
\hline $\begin{array}{l}\overrightarrow{2} \\
\stackrel{2}{-1} \\
\overrightarrow{4}\end{array}$ & 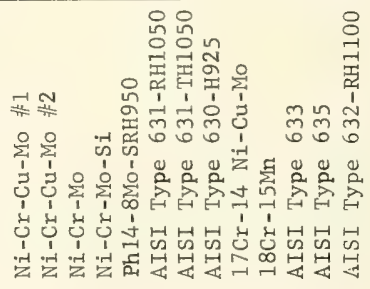 \\
\hline
\end{tabular}




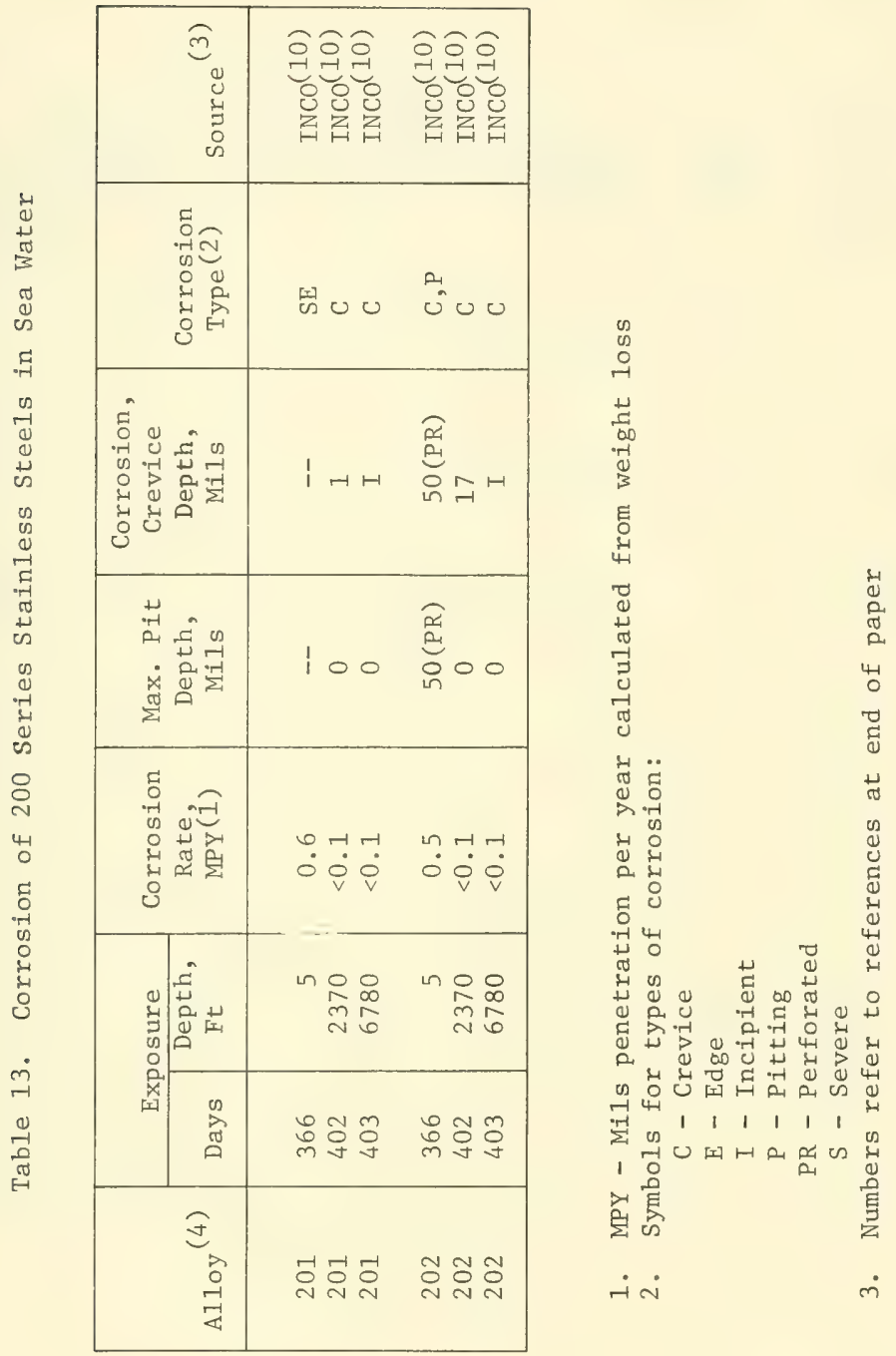




\begin{tabular}{|c|c|c|c|c|c|c|}
\hline 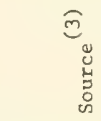 & 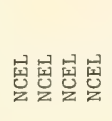 & 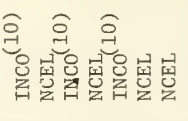 & 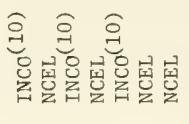 & 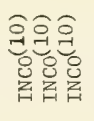 & 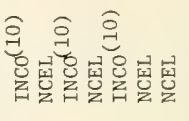 & 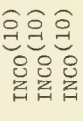 \\
\hline 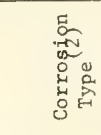 & 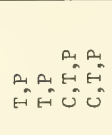 & 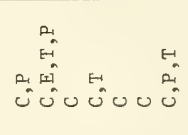 & 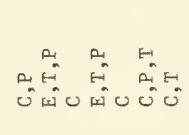 & in & 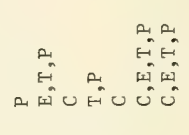 & 000 \\
\hline 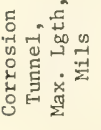 & 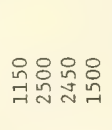 & 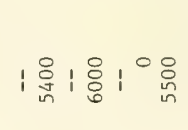 & 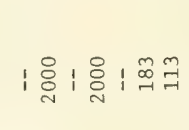 & $1: 11$ & 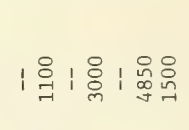 & $1: 11$ \\
\hline 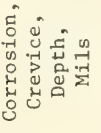 & 이배요 & H & m్mంH & 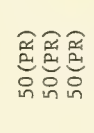 & OOHOH쓸 & HH \\
\hline 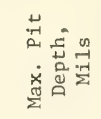 & 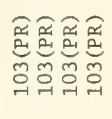 & 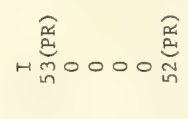 & 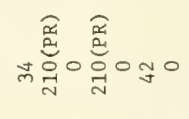 & 兑 & 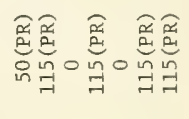 & 000 \\
\hline 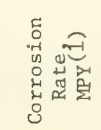 & iñ & 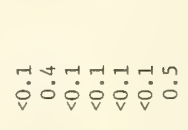 & 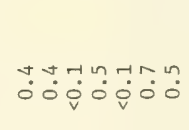 & Nion & 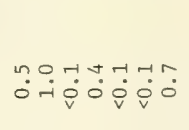 & ت1: \\
\hline 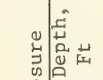 & 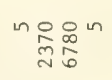 & 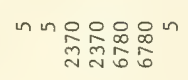 & 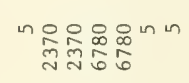 & 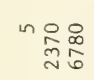 & 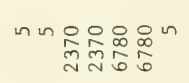 & 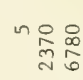 \\
\hline 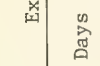 & 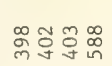 & 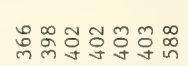 & 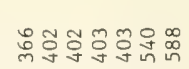 & 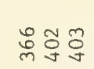 & 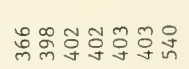 & 迎苧角 \\
\hline 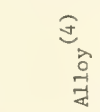 & 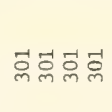 & 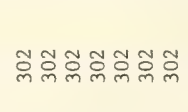 & 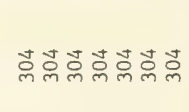 & 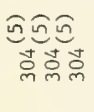 & 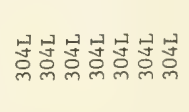 & த્లి \\
\hline
\end{tabular}




\begin{tabular}{|c|c|}
\hline 总 & 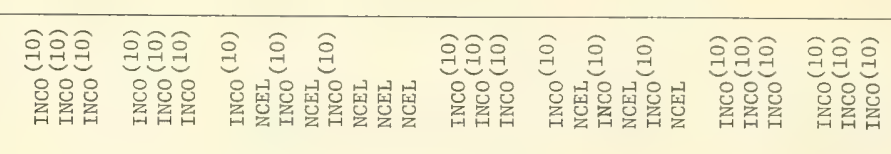 \\
\hline 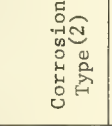 & 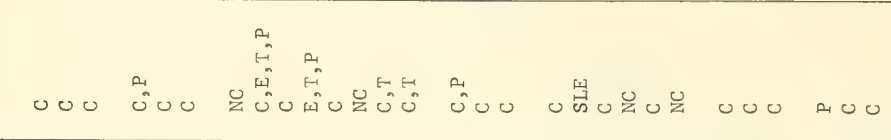 \\
\hline 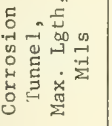 & 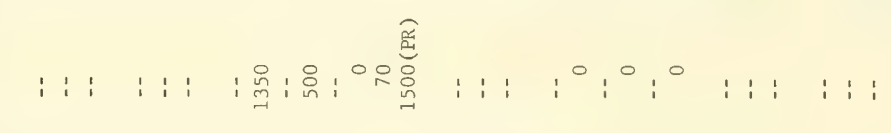 \\
\hline $\begin{array}{ll}0 \\
4 \\
4\end{array}$ & 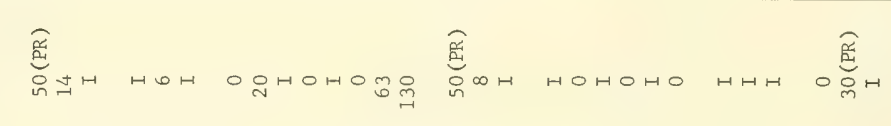 \\
\hline 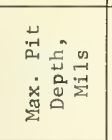 & 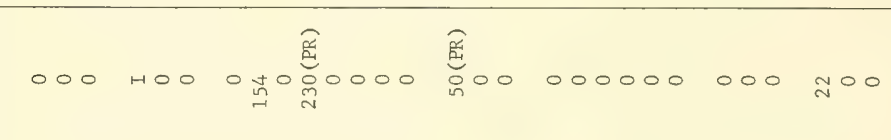 \\
\hline 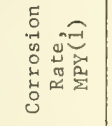 & 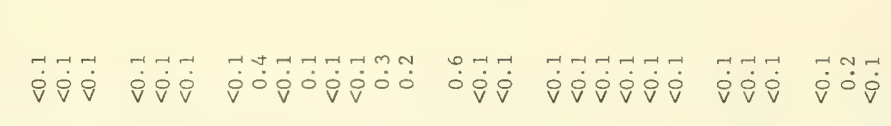 \\
\hline 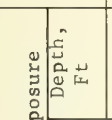 & 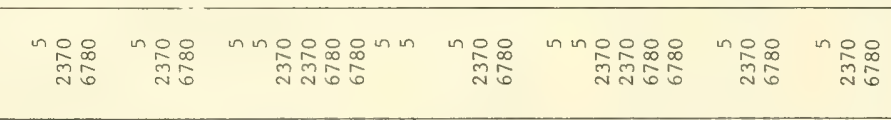 \\
\hline$\sqrt[2]{2}$ & 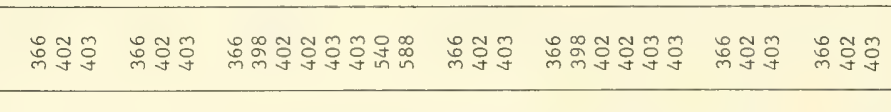 \\
\hline 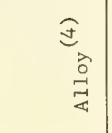 & 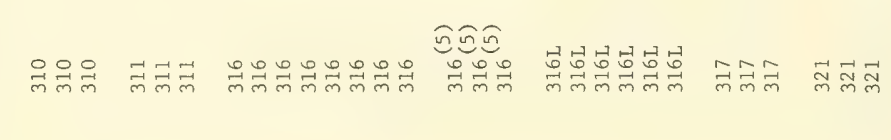 \\
\hline
\end{tabular}




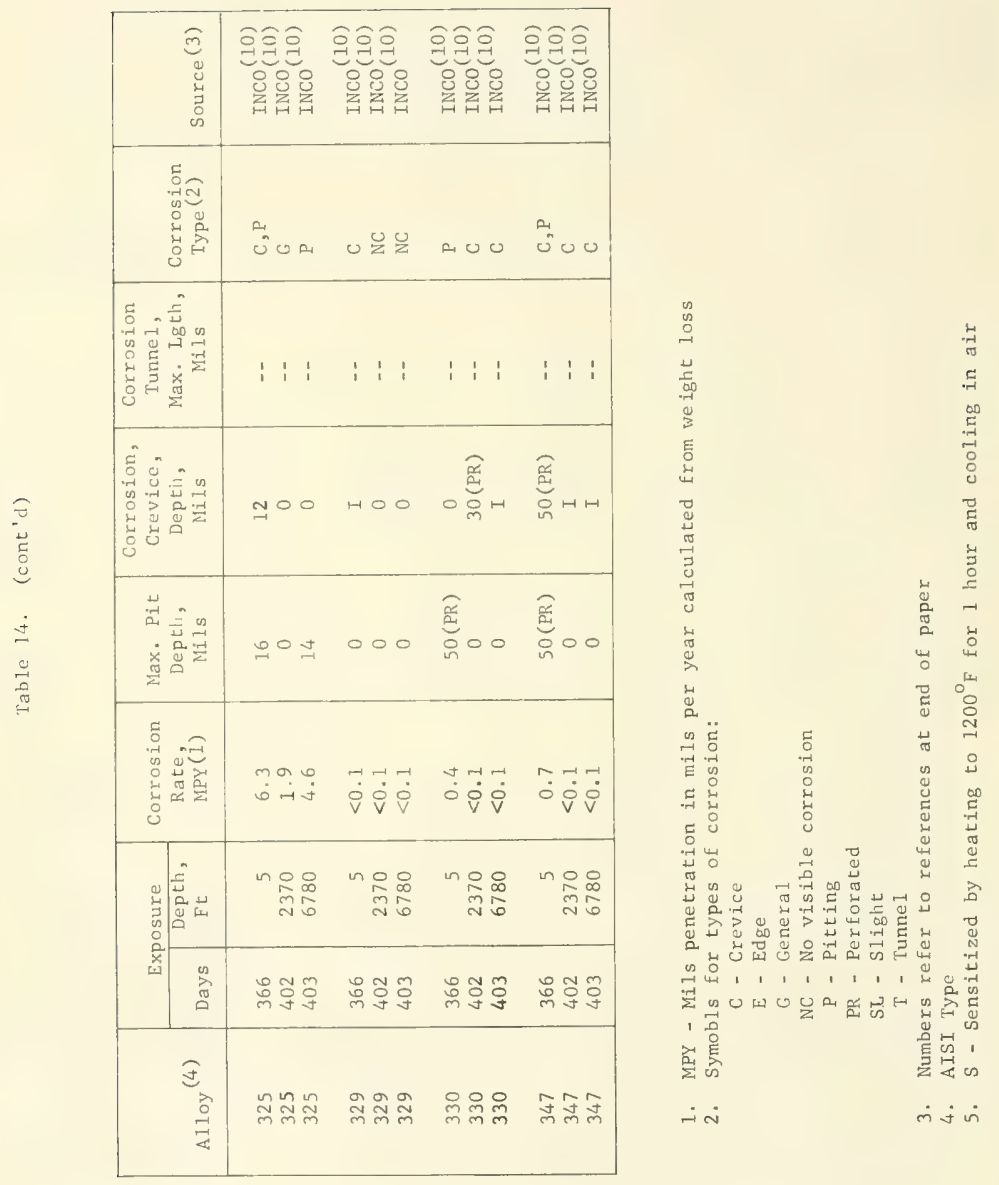




\begin{tabular}{|c|c|}
\hline 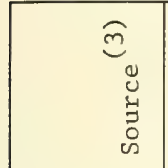 & 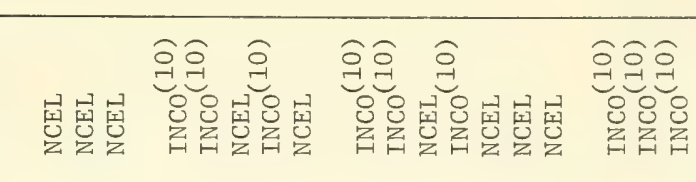 \\
\hline 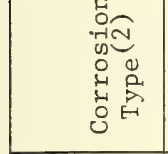 & 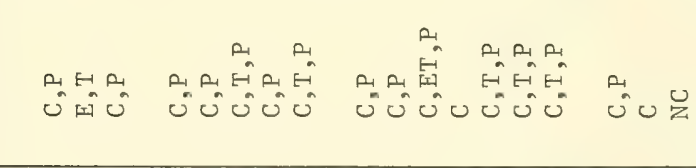 \\
\hline 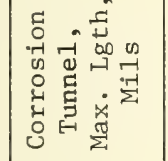 & 唔。 \\
\hline 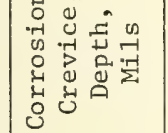 & 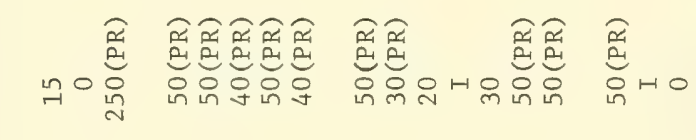 \\
\hline 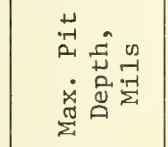 & 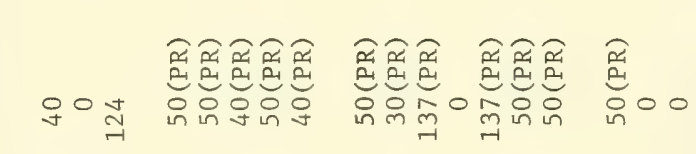 \\
\hline 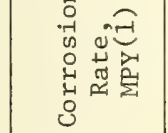 & 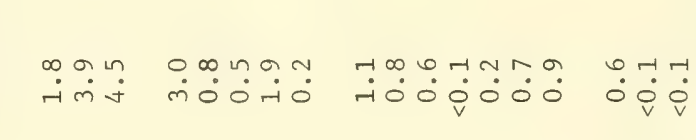 \\
\hline 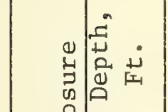 & 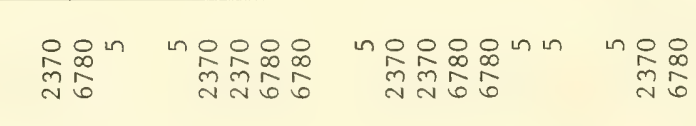 \\
\hline 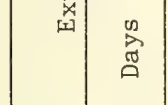 & 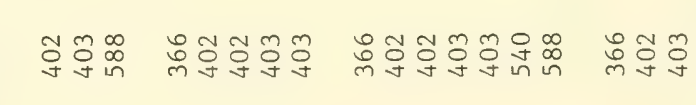 \\
\hline 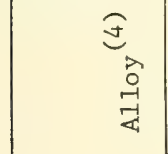 & 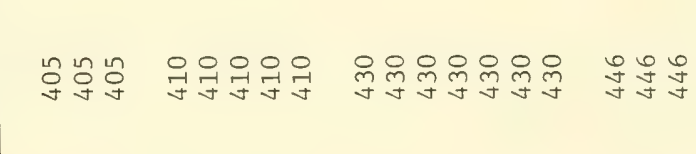 \\
\hline
\end{tabular}




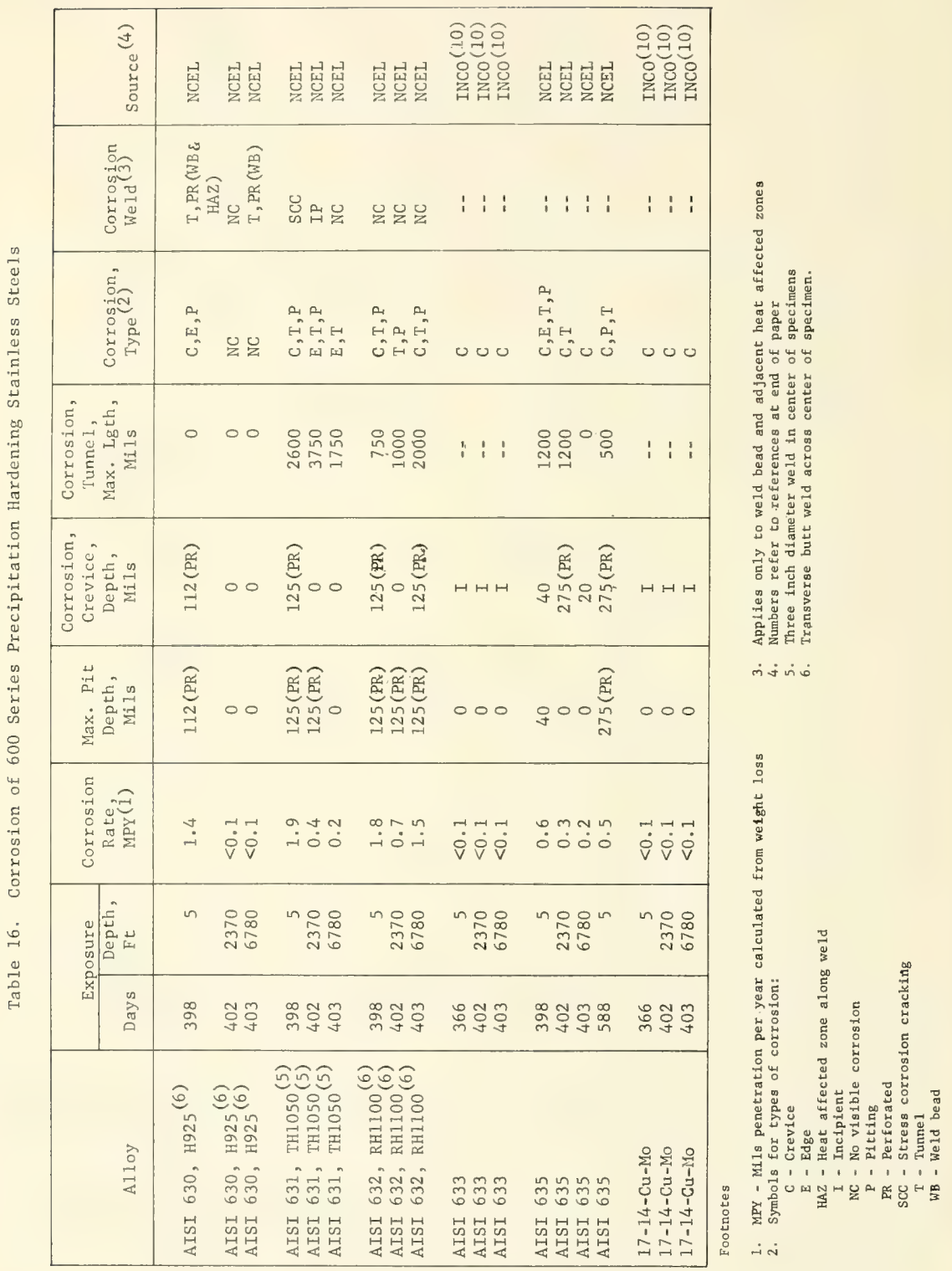




\begin{tabular}{|c|c|c|c|c|c|}
\hline 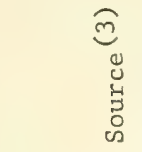 & 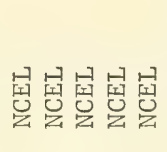 & 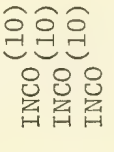 & 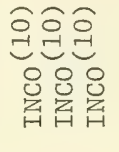 & 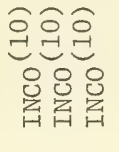 & 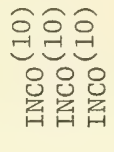 \\
\hline 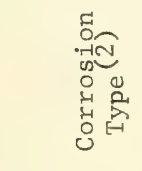 & 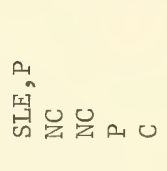 & 艺品 & ט O & $\cup م$ ه & 㟔ט0 \\
\hline 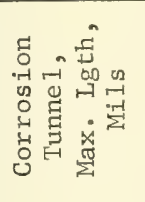 & 00000 & $1 i$ & $\begin{array}{ll}1 & 1 \\
1 & 1\end{array}$ & $\begin{array}{lll}1 & 1 & 1\end{array}$ & $\begin{array}{lll}i & 1 & 1\end{array}$ \\
\hline 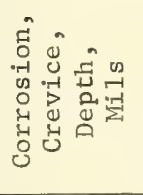 & $0000 \stackrel{\sim}{N}$ & $00 H$ & $H \infty 0$ & $\hat{N}^{\circ} 0$ & $O \mapsto H$ \\
\hline 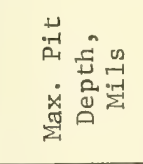 & ت্ন & $O H O$ & 000 & omo & 000 \\
\hline 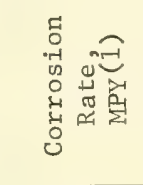 & 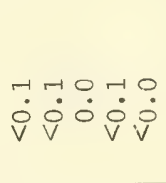 & $\begin{array}{l}\because-1 \\
\dot{0} \dot{0} \\
\dot{V}\end{array}$ & $\ddot{\circ} \dot{\circ} \dot{0} \dot{0}$ & $\ddot{0} \because \ddot{0}$ & $\ddot{\dot{0}} \dot{0} \dot{\nabla} \dot{\nabla}$ \\
\hline 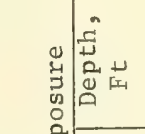 & 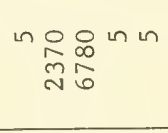 & 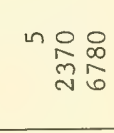 & $\begin{array}{l}\text { n } 0 \\
\text { m } \\
\text { N }\end{array}$ & 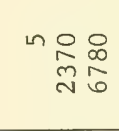 & 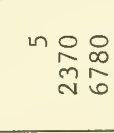 \\
\hline 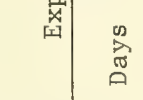 & 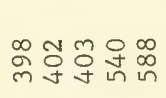 & 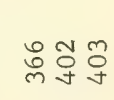 & 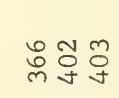 & 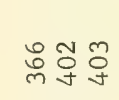 & 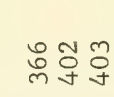 \\
\hline 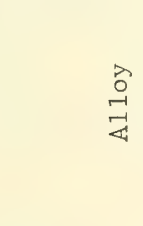 & 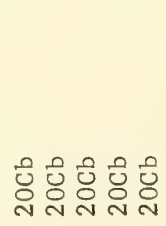 & $\begin{array}{lll}m & m & m \\
1 & 1 & 1 \\
0 & 0 & 0 \\
0 & 0 & 0 \\
0 & 0 & 0 \\
\text { N } & N\end{array}$ & 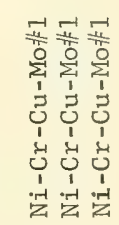 & 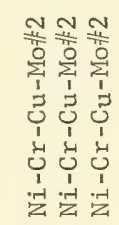 & 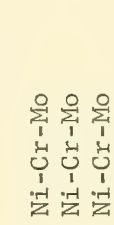 \\
\hline
\end{tabular}




\begin{tabular}{|c|c|c|c|}
\hline 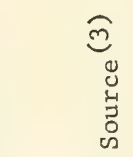 & 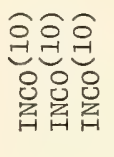 & 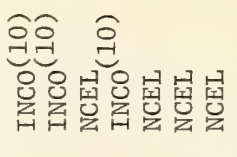 & \multirow{9}{*}{ 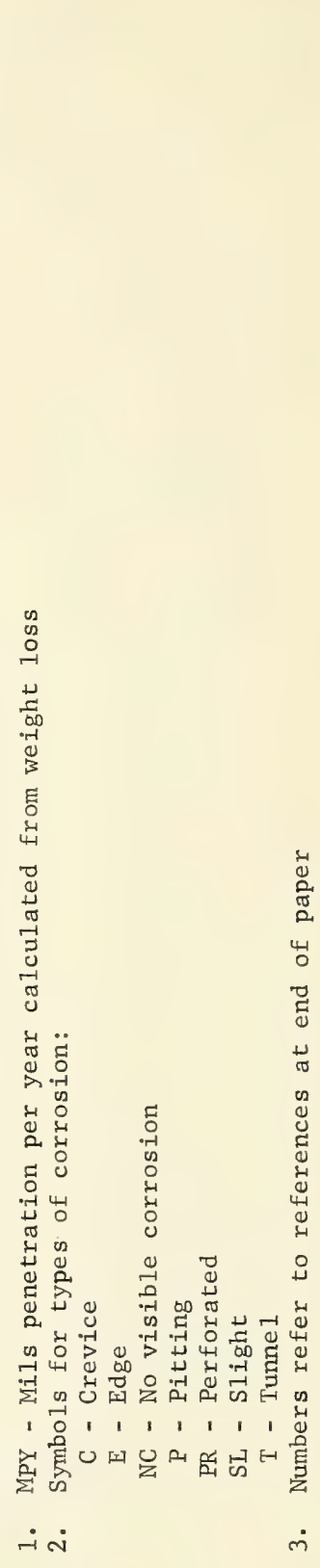 } \\
\hline 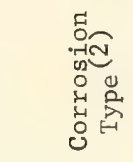 & 吕吕吕 & 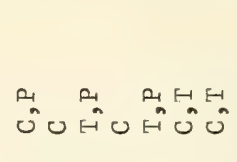 & \\
\hline 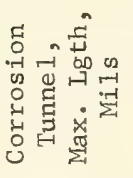 & 1 i i & 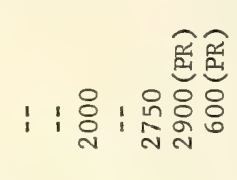 & \\
\hline 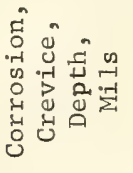 & 000 & 危点 & \\
\hline 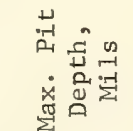 & 000 & 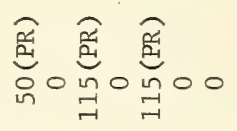 & \\
\hline 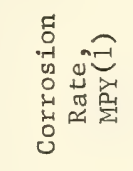 & $\ddot{\circ} \dot{\nabla} \dot{\theta} \dot{\circ}$ & 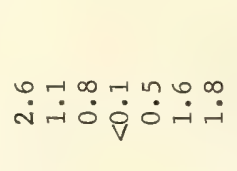 & \\
\hline ن. & 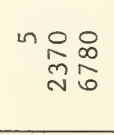 & 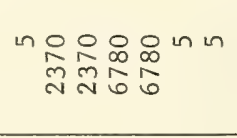 & \\
\hline 되 & 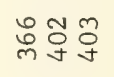 & 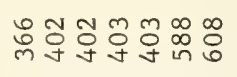 & \\
\hline $\begin{array}{l}\hat{2} \\
\stackrel{-1}{=} \\
\stackrel{-1}{4}\end{array}$ & 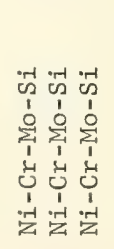 & 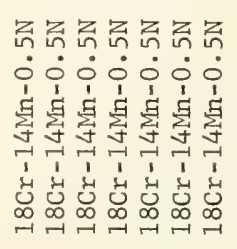 & \\
\hline
\end{tabular}




\begin{tabular}{|c|c|c|c|c|c|}
\hline $\begin{array}{c}\text { తิ } \\
0 \\
u \\
y \\
0 \\
0\end{array}$ & 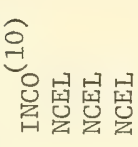 & 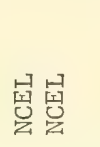 & 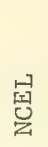 & 点 & \\
\hline 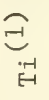 & 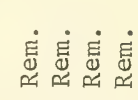 & 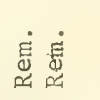 & $\underset{\dot{\Xi}}{\dot{\Xi}}$ & $\underset{\mathscr{E}}{\stackrel{\Xi}{凶}}$ & \\
\hline 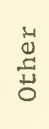 & $1: 1 \frac{ \pm}{1}$ & 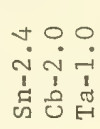 & $i$ & $i$ & \\
\hline ठै & 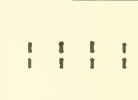 & $i$ & $!$ & @ & \\
\hline$>$ & $i \quad i \quad i$ & $i$ & $\begin{array}{l}0 \\
\dot{j}\end{array}$ & $\dot{0}$ & \\
\hline$\stackrel{-1}{\varangle}$ & 1 i i i & 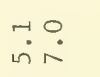 & in & $\stackrel{\circ}{\circ}$ & \\
\hline 0 & $1: \stackrel{0}{1}$ & $\begin{array}{l}\infty \\
\stackrel{\infty}{+} \\
\dot{0} \\
0\end{array}$ & ت्! & $\stackrel{\sim}{\sim}$ & \\
\hline I & 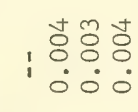 & $\begin{array}{ll}\infty & N \\
\wp & 8 \\
0 & 0 \\
0 & 0\end{array}$ & $\begin{array}{l}\hat{8} \\
0 \\
0\end{array}$ & $\begin{array}{l}0 \\
\stackrel{1}{0} \\
\dot{0}\end{array}$ & $\begin{array}{l}\text { aे } \\
\text { बे } \\
\text { बे }\end{array}$ \\
\hline$z$ & 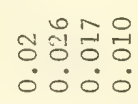 & $\begin{array}{l}m \\
\begin{array}{l}1 \\
0\end{array} \\
0 \\
0\end{array}$ & \begin{tabular}{l} 
\pm \\
\multirow{0}{0}{} \\
0
\end{tabular} & $\begin{array}{l}\tilde{N} \\
0 \\
0\end{array}$ & 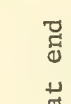 \\
\hline (1) & 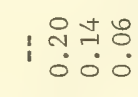 & $\begin{array}{l}N \text { ? } \\
\text { mo } \\
0\end{array}$ & $\stackrel{\sim}{\because}$ & $\stackrel{+}{\overrightarrow{1}}$ & 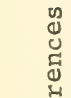 \\
\hline 0 & 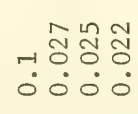 & 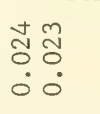 & $\begin{array}{l}\text { ָे } \\
0 \\
0\end{array}$ & $\begin{array}{l}\text { 거 } \\
\text { ல } \\
0\end{array}$ & 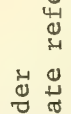 \\
\hline 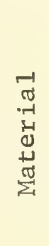 & 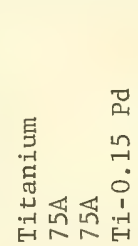 & 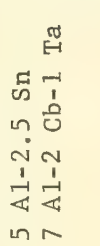 & $\begin{array}{l}> \\
+ \\
1 \\
1 \\
-1 \\
0 \\
0\end{array}$ & $\begin{array}{l}-1 \\
4 \\
m \\
1 \\
4 \\
0 \\
-1 \\
-1 \\
1 \\
m \\
\text { m }\end{array}$ & 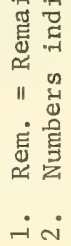 \\
\hline
\end{tabular}




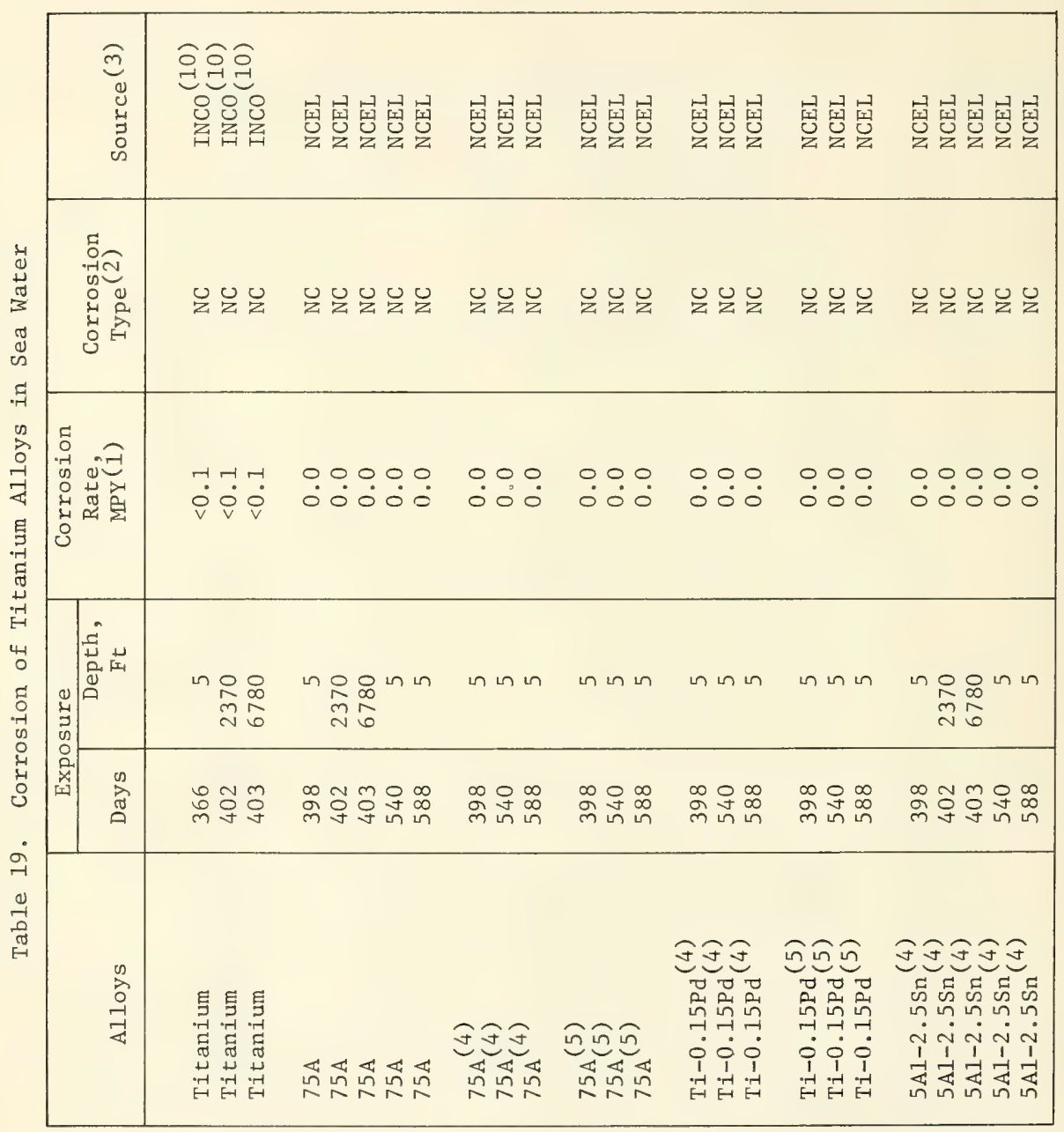




\begin{tabular}{|c|c|c|c|c|c|c|}
\hline $\begin{array}{c}\widehat{0} \\
0 \\
\breve{~} \\
0 \\
0\end{array}$ & 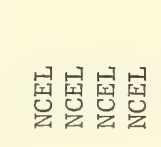 & 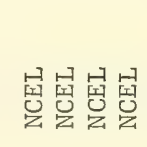 & 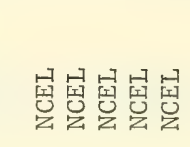 & 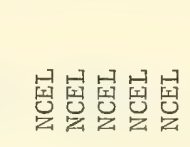 & 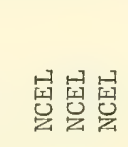 & 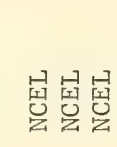 \\
\hline 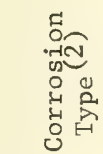 & 吕号克呈 & 只虽号品 & 兄兄兄克兄 & 兄兄吕兄兄 & 号号品 & 号号虽 \\
\hline 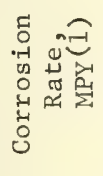 & $\begin{array}{l}000 \\
\dot{0} \dot{0}: \dot{0}\end{array}$ & 웅우 & 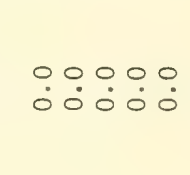 & 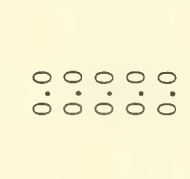 & $\begin{array}{l}0 \\
\dot{0} \\
0\end{array}$ & $\begin{array}{l}0: 0 \\
000 \\
0\end{array}$ \\
\hline 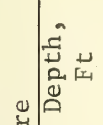 & 음요 & 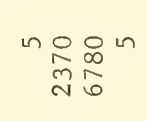 & n & 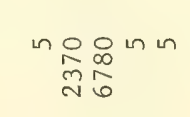 & $\ln n \operatorname{nn}$ & $\operatorname{nn} \backsim \backsim$ \\
\hline 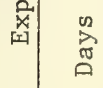 & 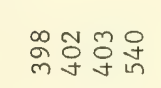 & 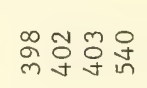 & 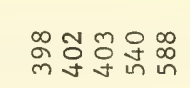 & 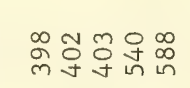 & 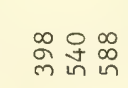 & 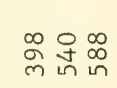 \\
\hline 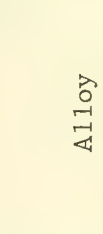 & 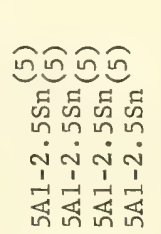 & 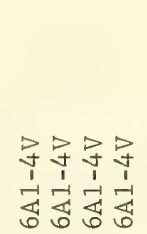 & 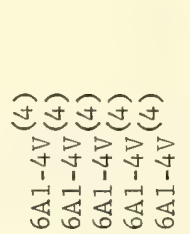 & 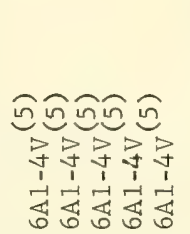 & 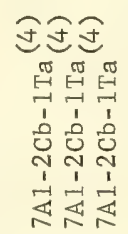 & 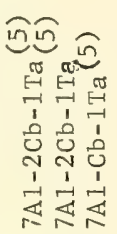 \\
\hline
\end{tabular}




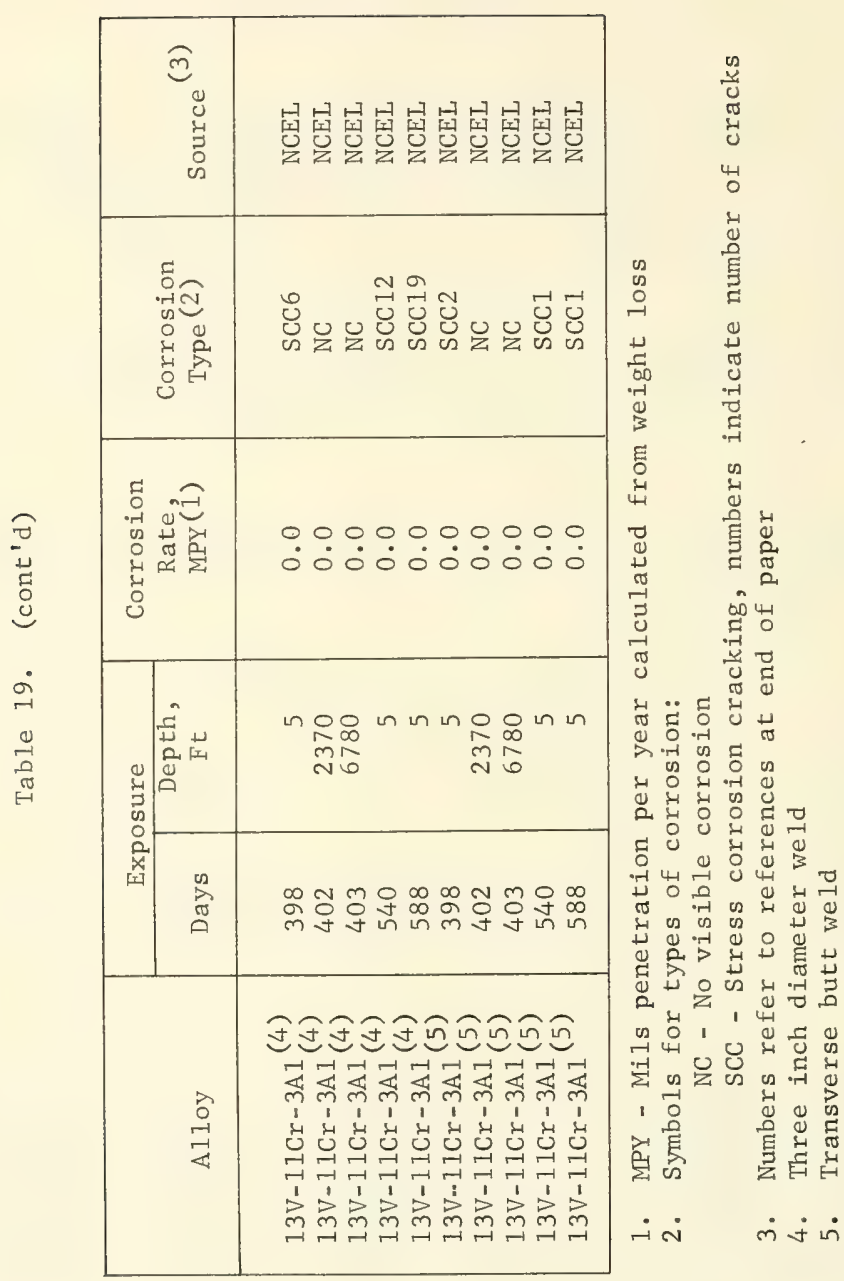




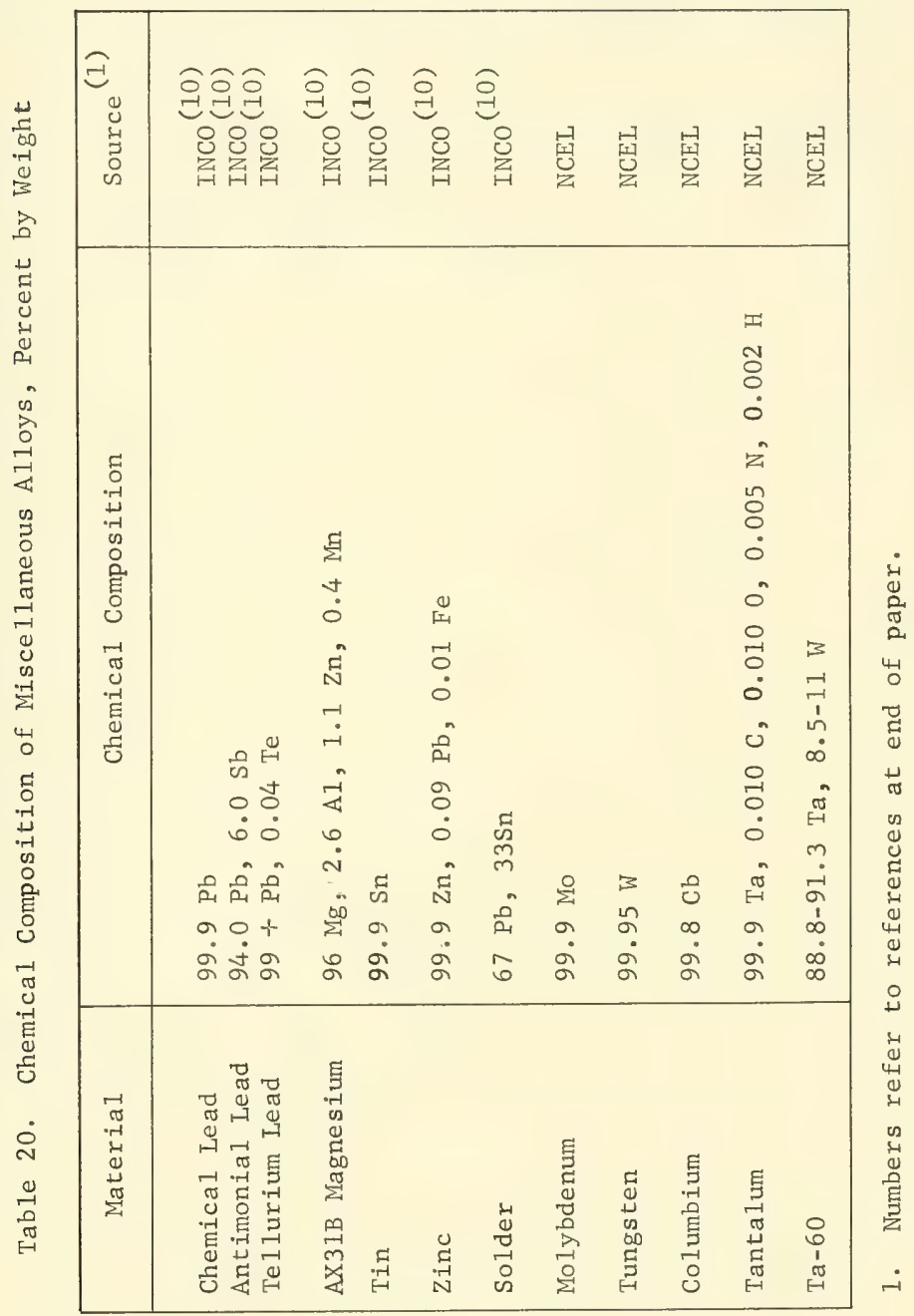




\begin{tabular}{|c|c|c|c|c|c|c|c|c|}
\hline 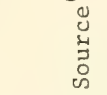 & 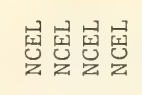 & 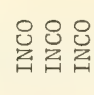 & $\begin{array}{l}80 \\
\text { 足是 }\end{array}$ & 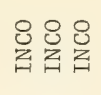 & 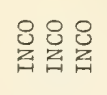 & 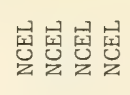 & 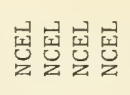 & 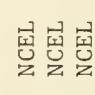 \\
\hline 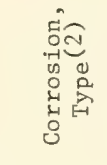 & 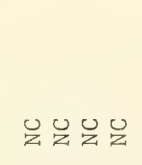 & $D D$ & $D D$ & D & さきす & ن & 乬乬乬 & $\dot{z} \tilde{z}$ \\
\hline 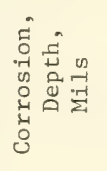 & ：１１１１ & 111 & 111 & 111 & 111 & or : & $1 \quad 1 \quad 1$ & $1: 1$ \\
\hline 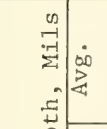 & $1 \quad 1 \quad 1 \quad 1$ & 111 & $1 \quad 1 \quad 1$ & $1 \quad 11$ & $1 \quad 1$ & $\begin{array}{llll}0 & 1 & 1 & 1\end{array}$ & 1111 & 111 \\
\hline 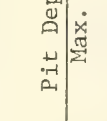 & $\begin{array}{llll}1 & 1 & 1 & 1\end{array}$ & 111 & 111 & 111 & 乘乘 & 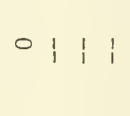 & $\begin{array}{llll}1 & 1 & 1 & 1\end{array}$ & 111 \\
\hline 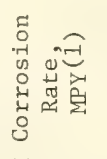 & $\begin{array}{l}8: 8: 8 \\
0000\end{array}$ & :? & 茴 & थீ & 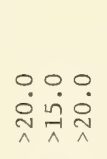 & ت- & $\begin{array}{l}8: 8: 8 \\
0000\end{array}$ & $\begin{array}{l}8: 8 \\
0: 0 \\
00\end{array}$ \\
\hline 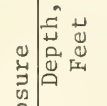 & no & n尺 & 요요 & 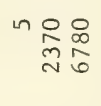 & n。요 & in $\underset{N}{\stackrel{v}{N}}$ in in & n $\underset{N}{N} \min$ & $\ln i n \in$ \\
\hline 齐 & 忒 & 串尽 & 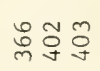 & 율웅 & 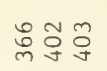 & 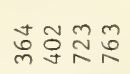 & 矛尽 & 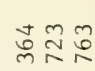 \\
\hline $\begin{array}{l}\hat{0} \\
\text { 妾 }\end{array}$ & 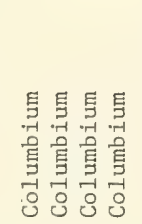 & 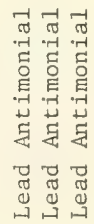 & 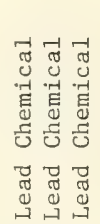 & 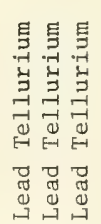 & 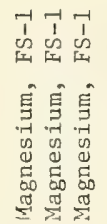 & 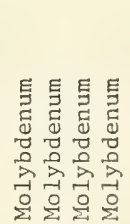 & 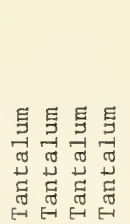 & 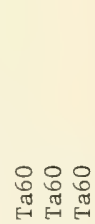 \\
\hline
\end{tabular}




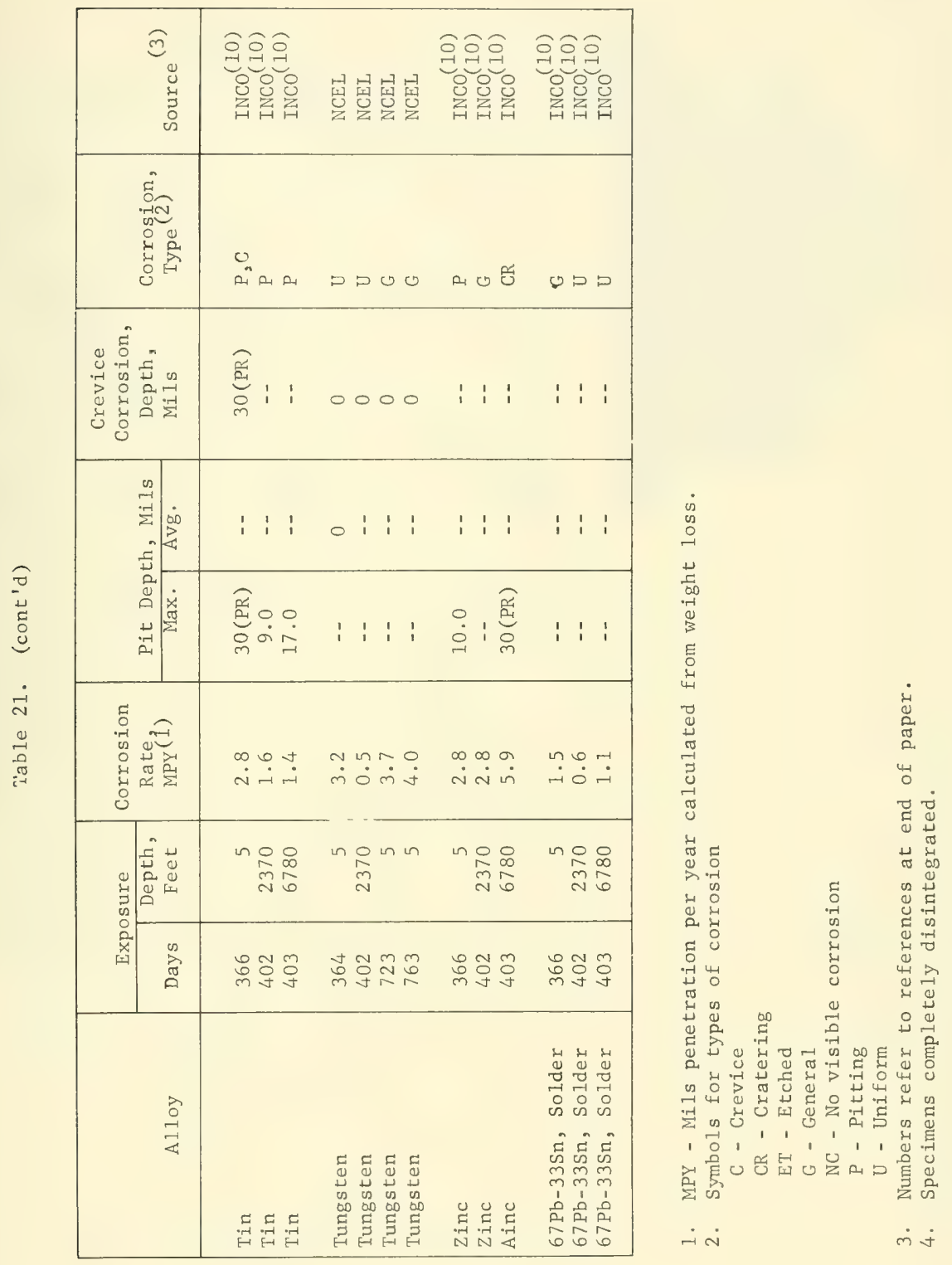




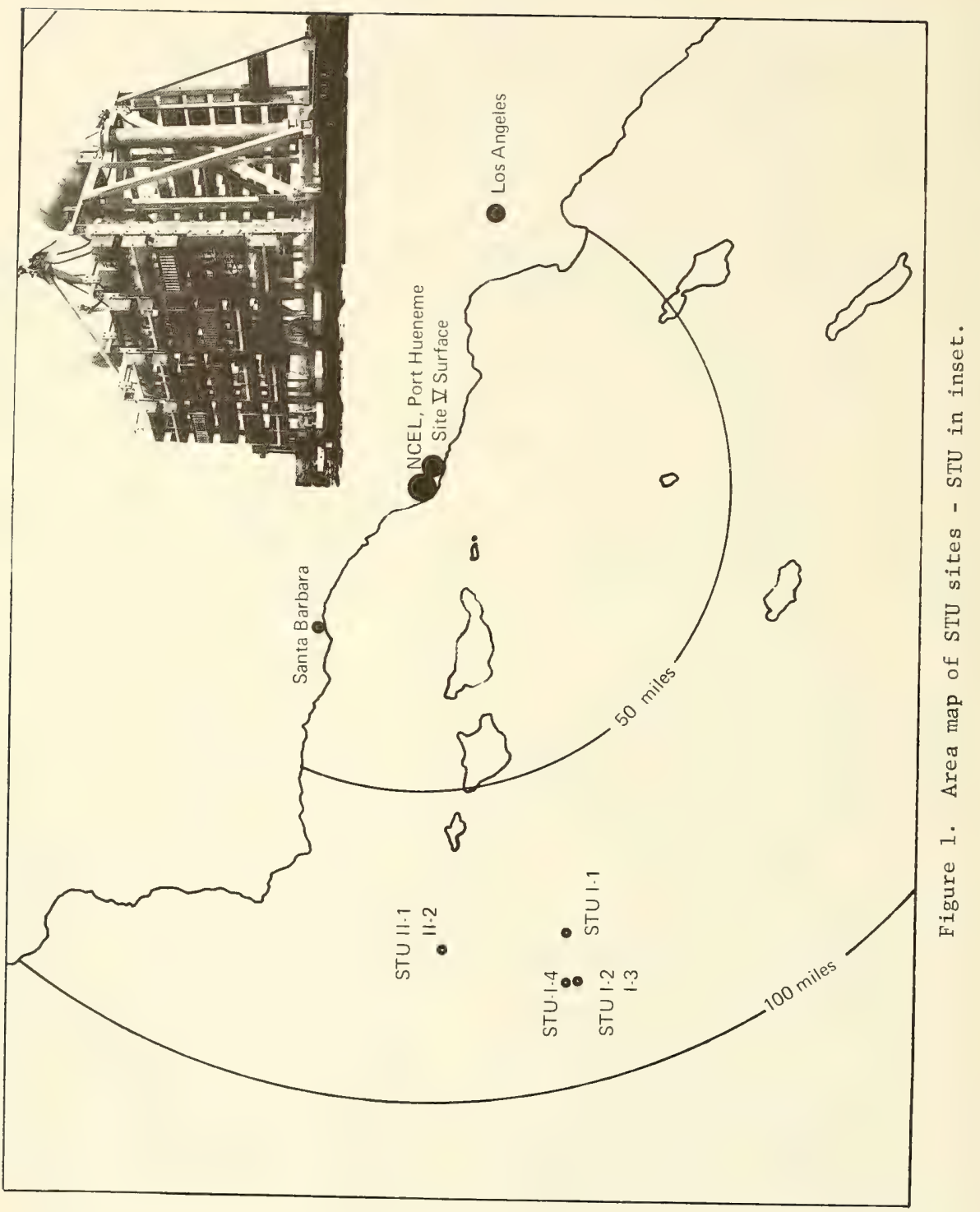




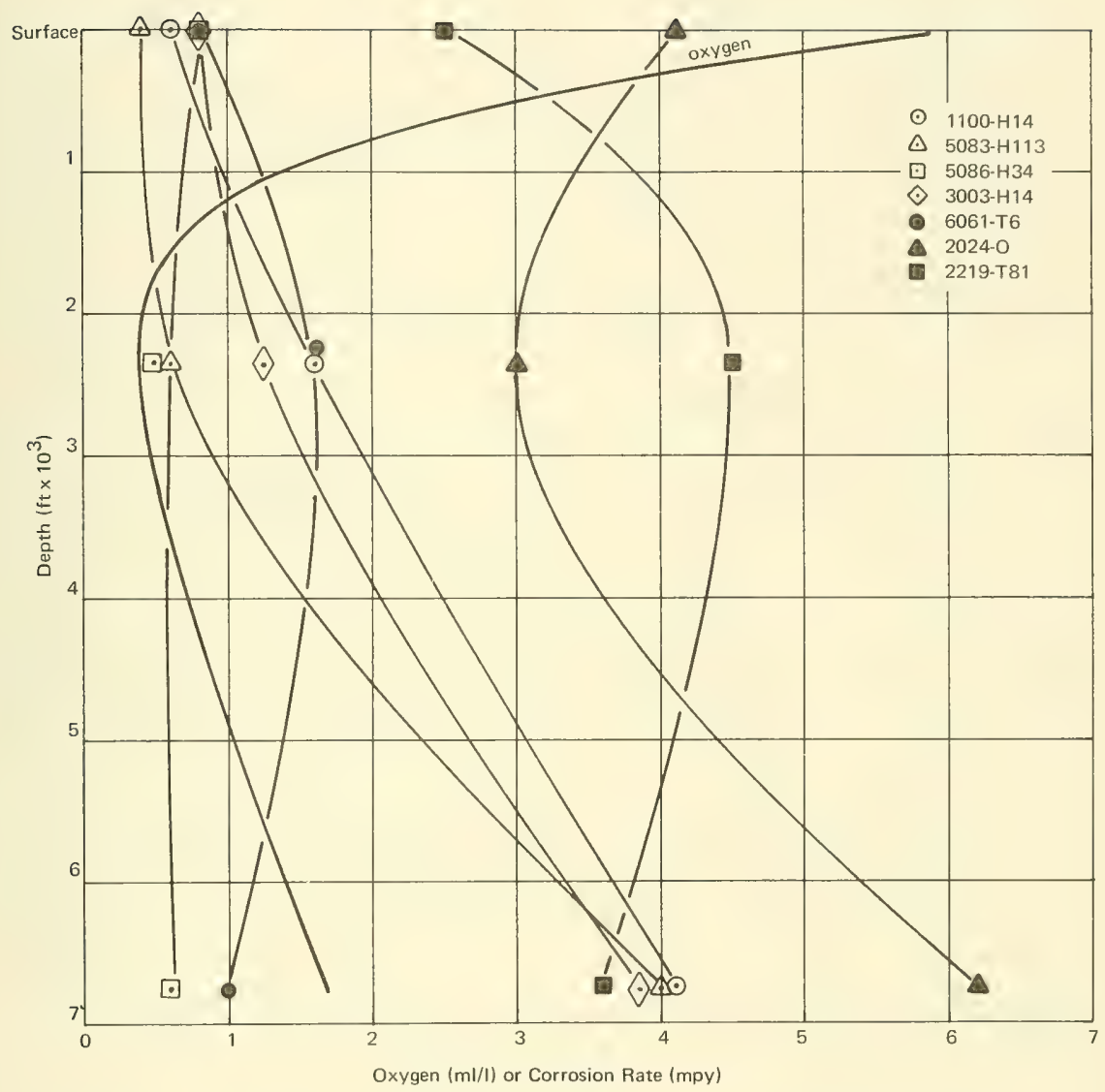

Figure 2. Corrosion rates of aluminum alloys vs depth after 1 year of exposure. 


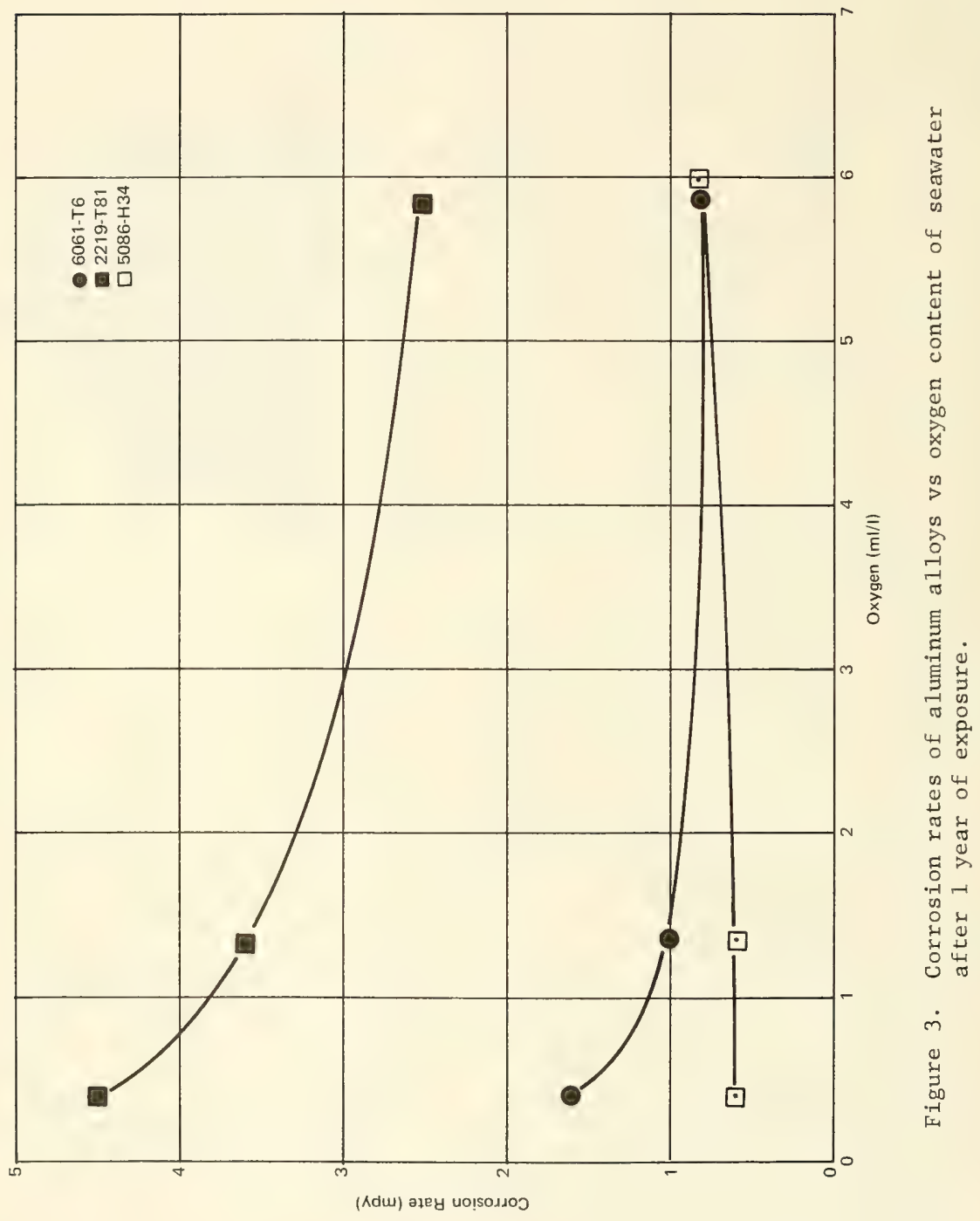




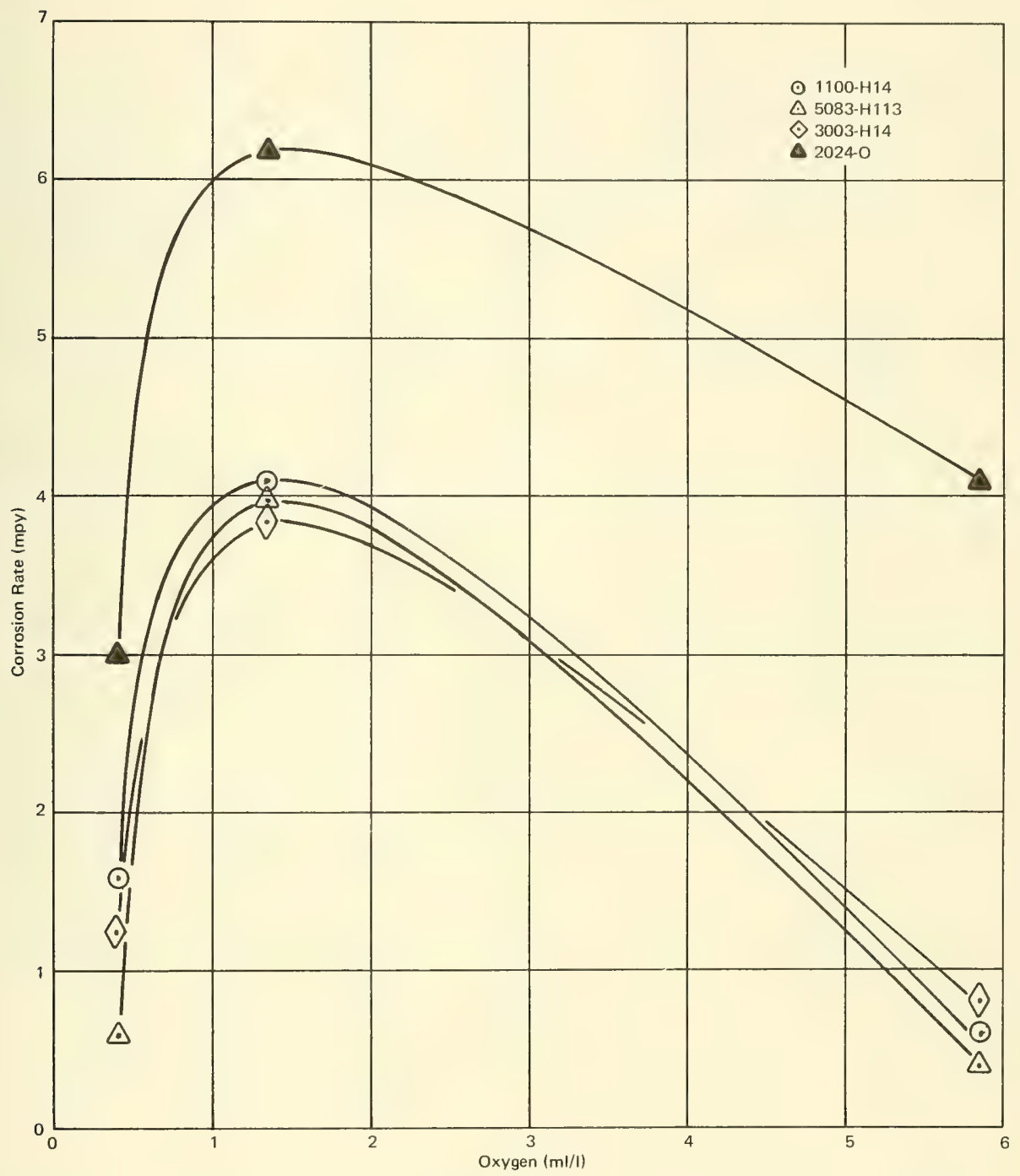

Figure 4. Corrosion rates of aluminum alloys vs oxygen content of seawater after 1 year of exposure. 


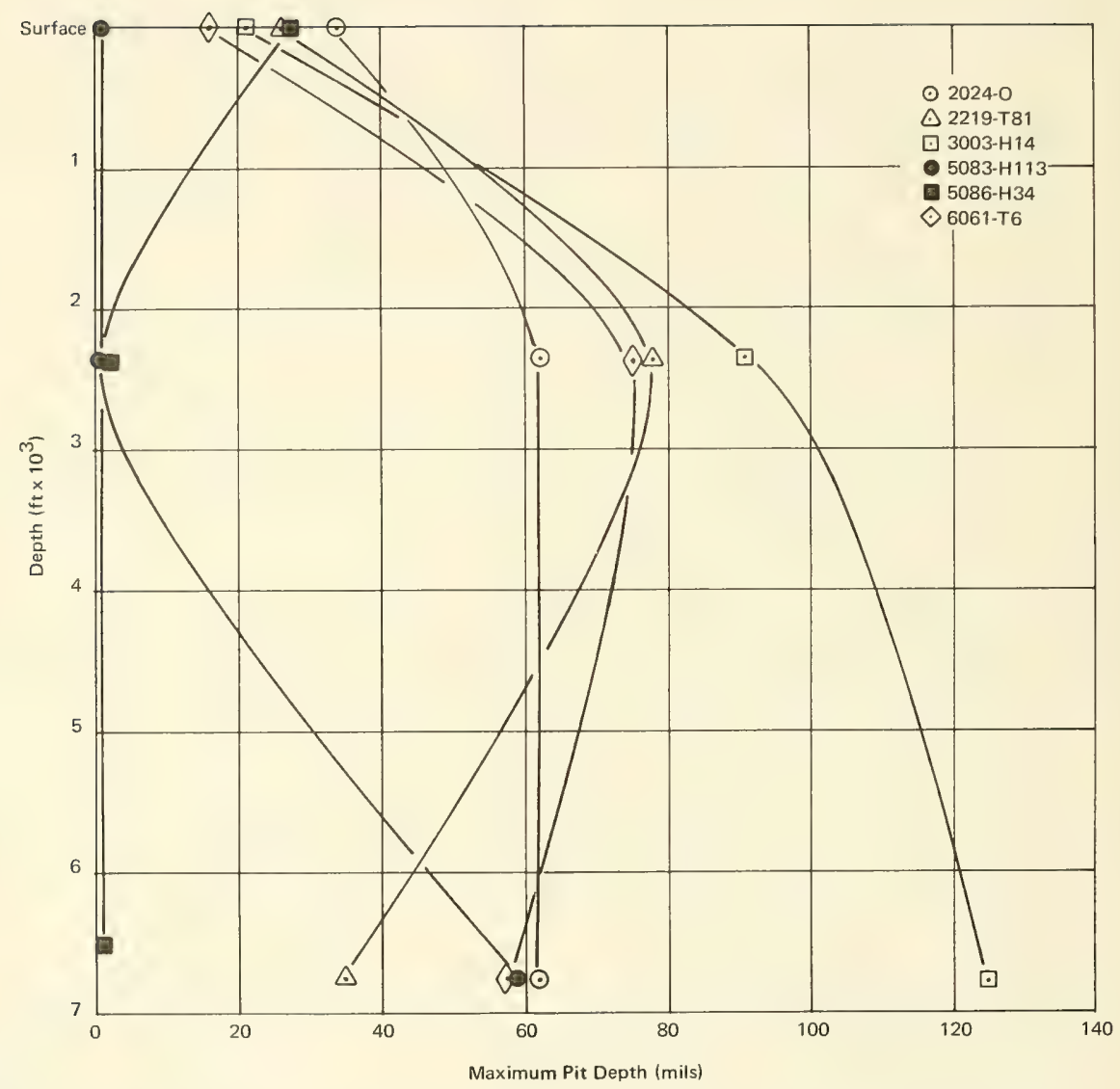

Figure 5. Maximum depths of pits of aluminum alloys vs depth after 1 year of exposure. 


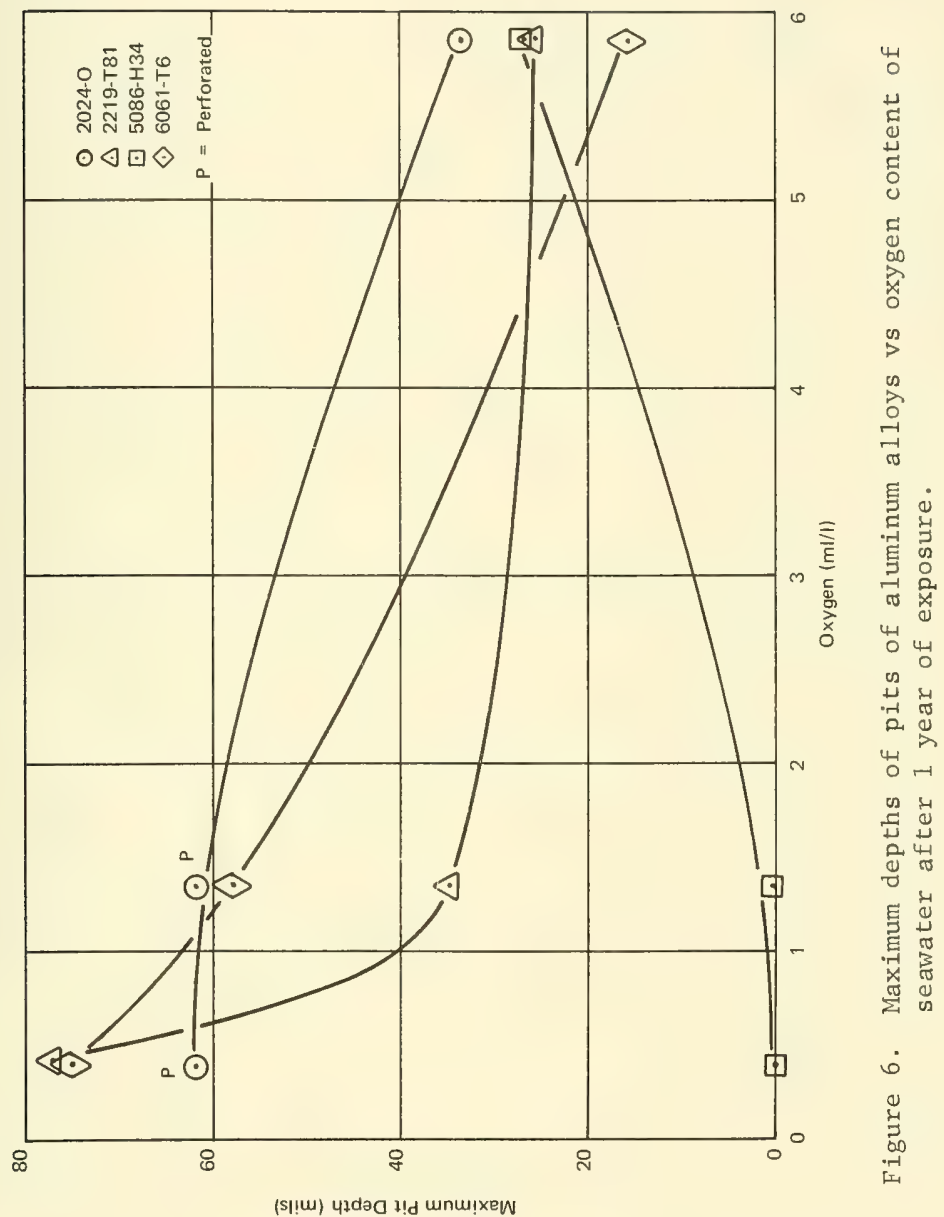

(s!!u) 4zdag t!d unum!xeW 


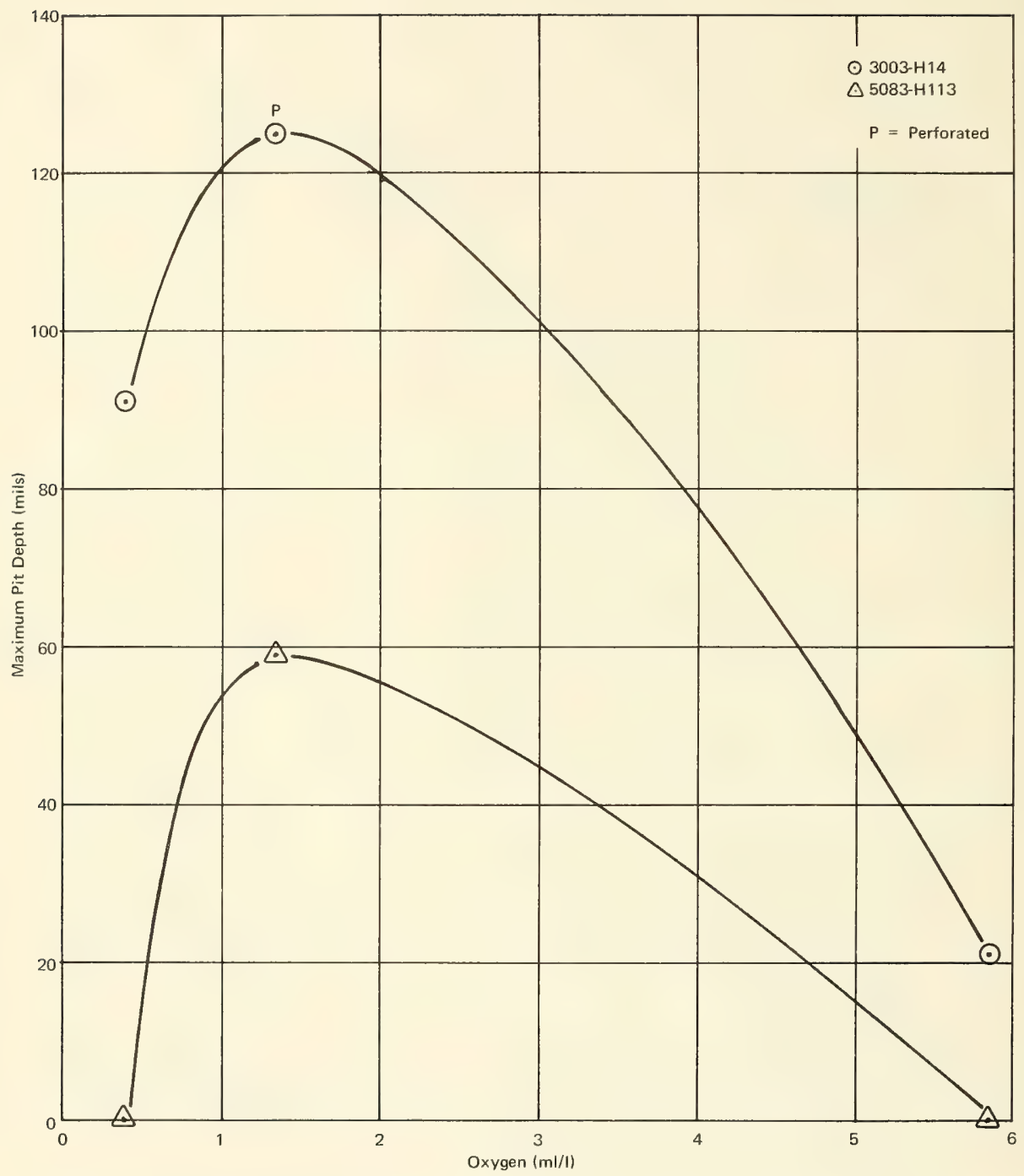

Figure 7. Maximum depths of pits of aluminum alloys vs oxygen content of seawater after 1 year of exposure. 


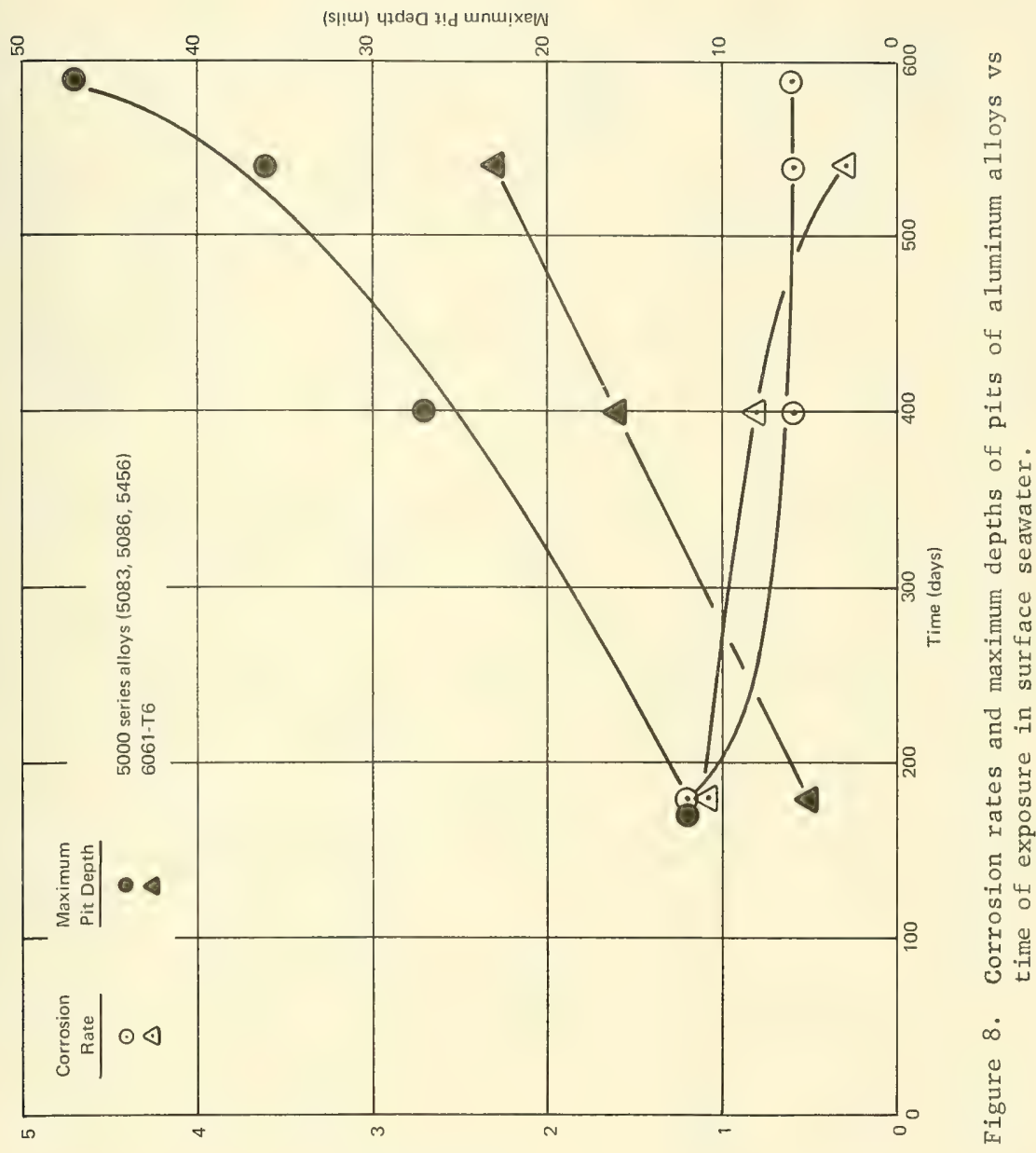

(^dw) әъеу uо!sodsoว 


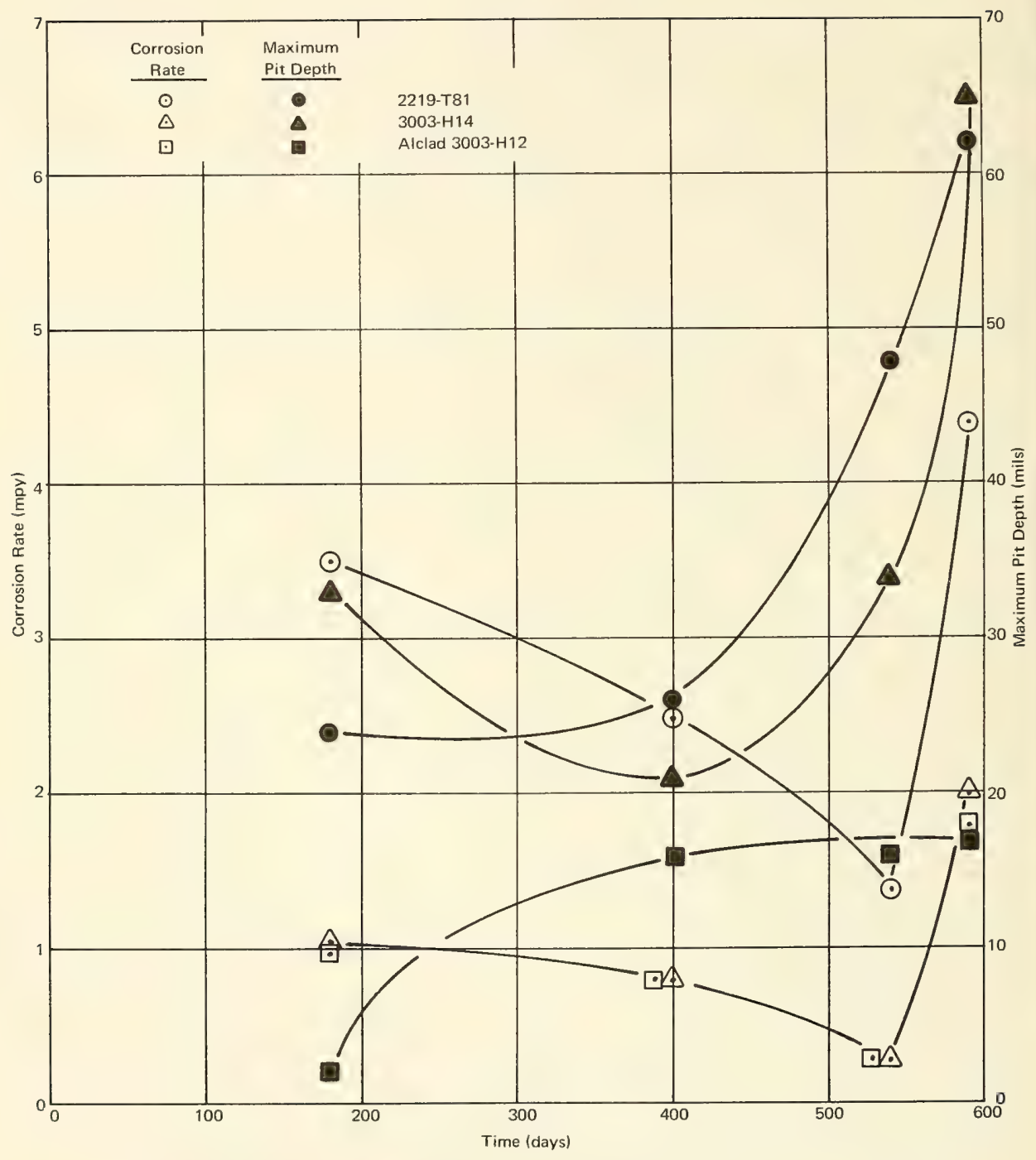

Figure 9. Corrosion rates and maximum depths of pits of aluminum alloys vs time of exposure in surface seawater. 


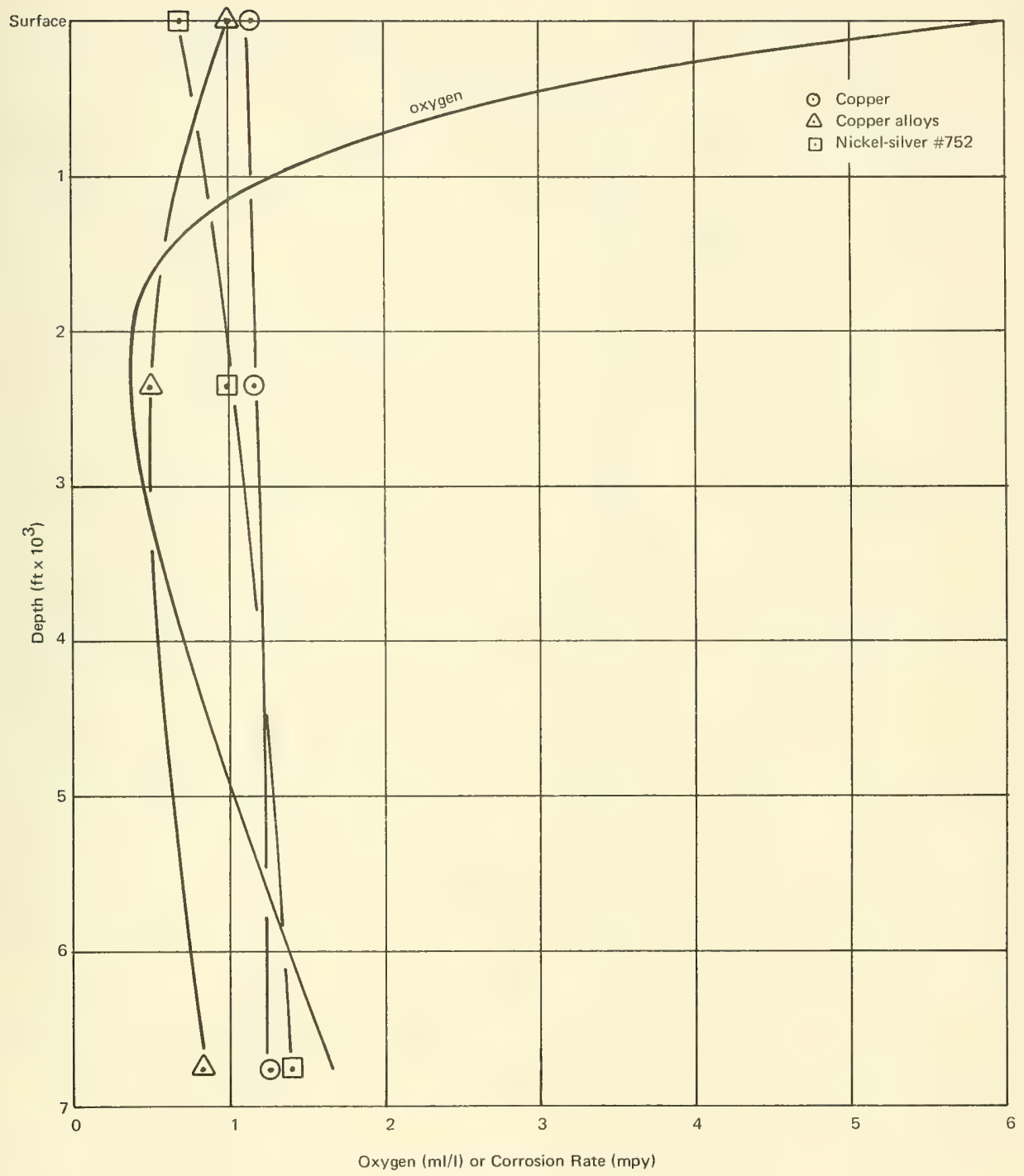

Figure 10. Corrosion of copper alloys vs depth after 1 year of exposure. 


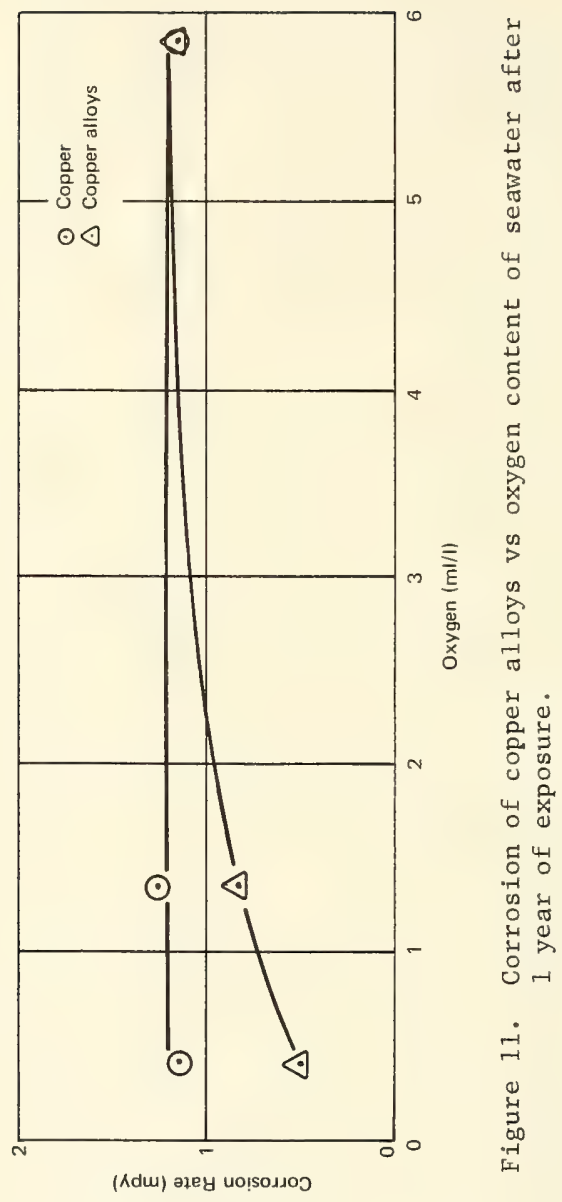




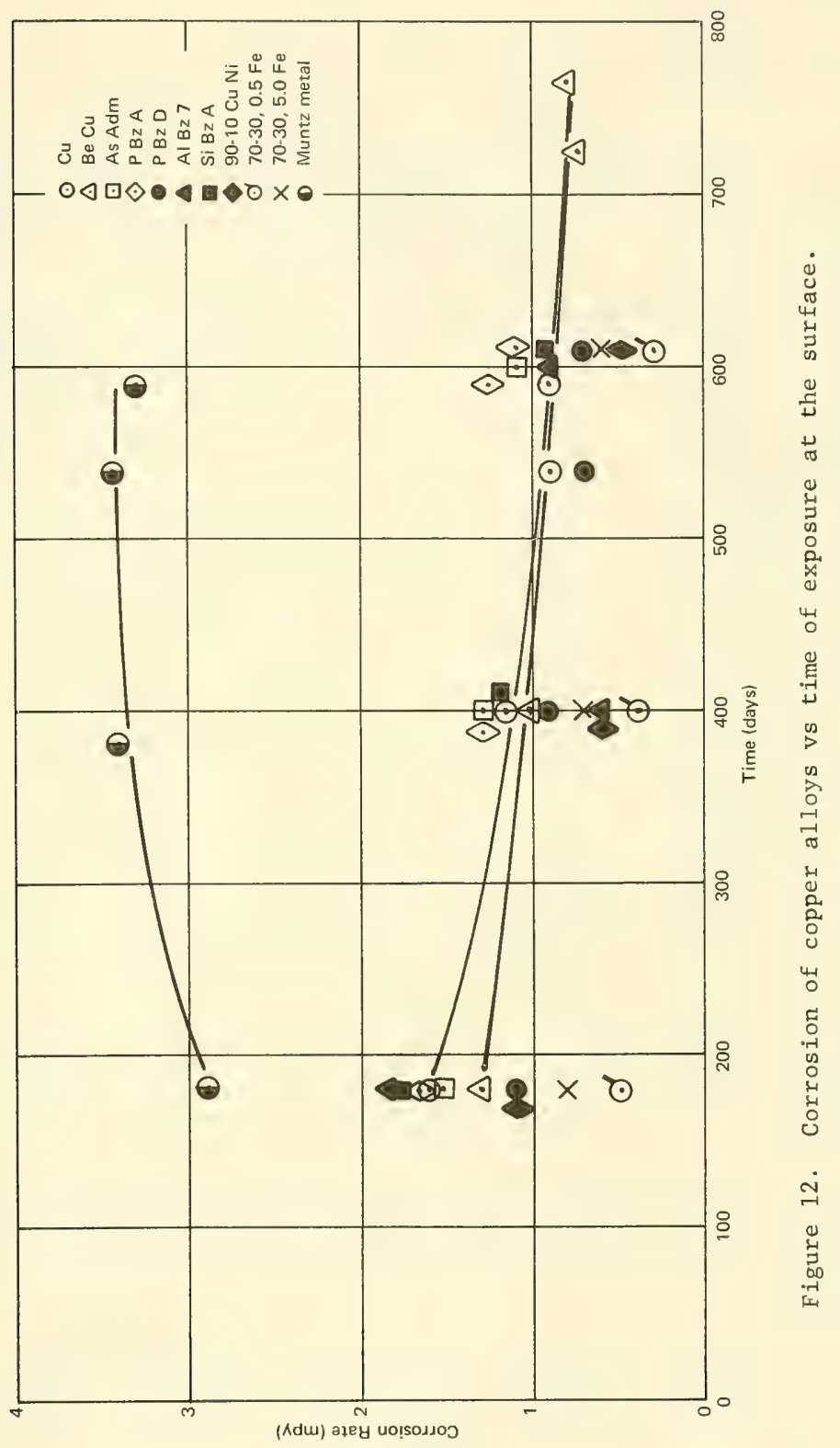




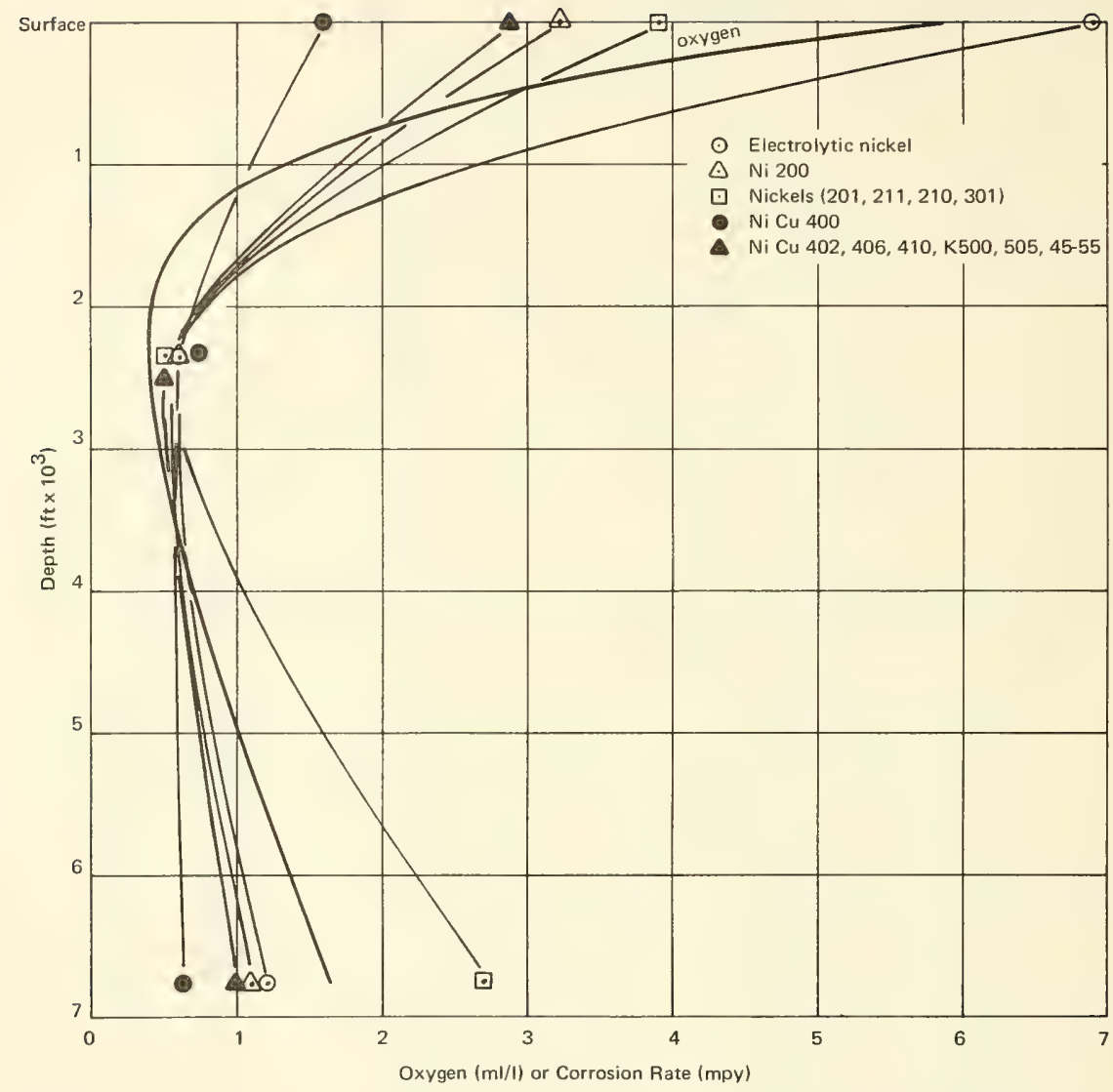

Figure 13. Corrosion of nickels and nickel-copper alloys vs depth after 1 year of exposure. 


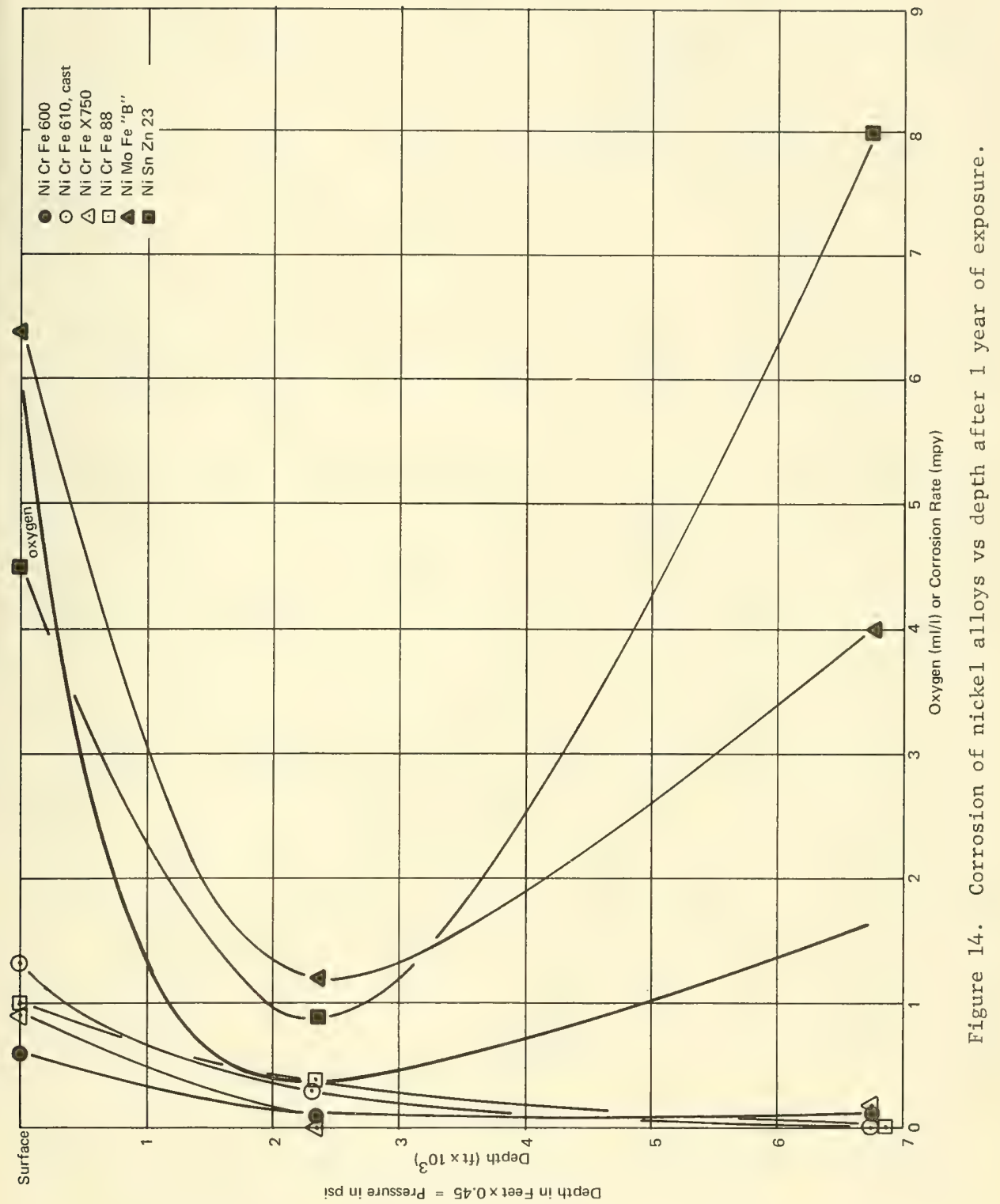




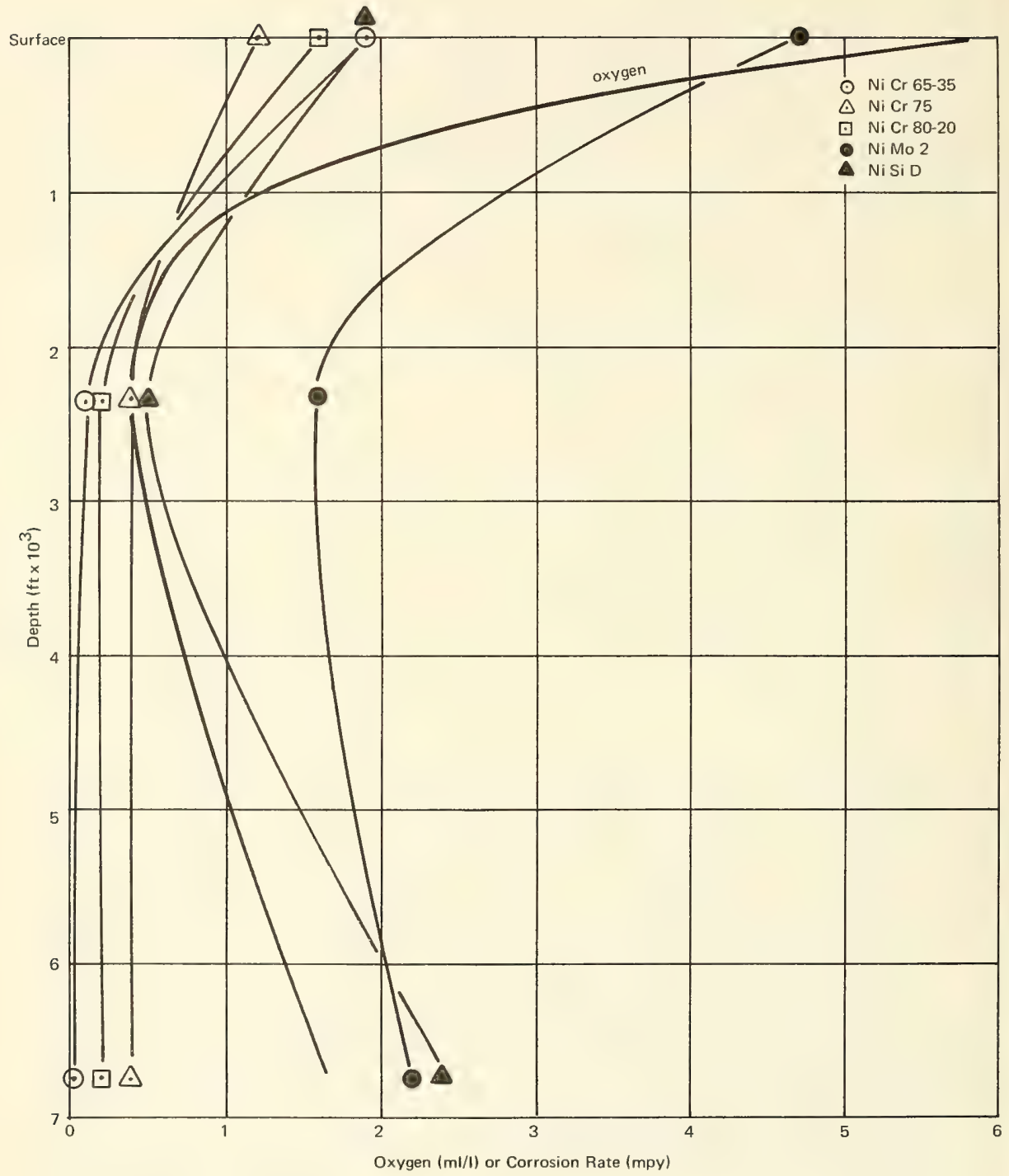

Figure 15. Corrosion of nickel alloys vs depth after 1 year of exposure. 


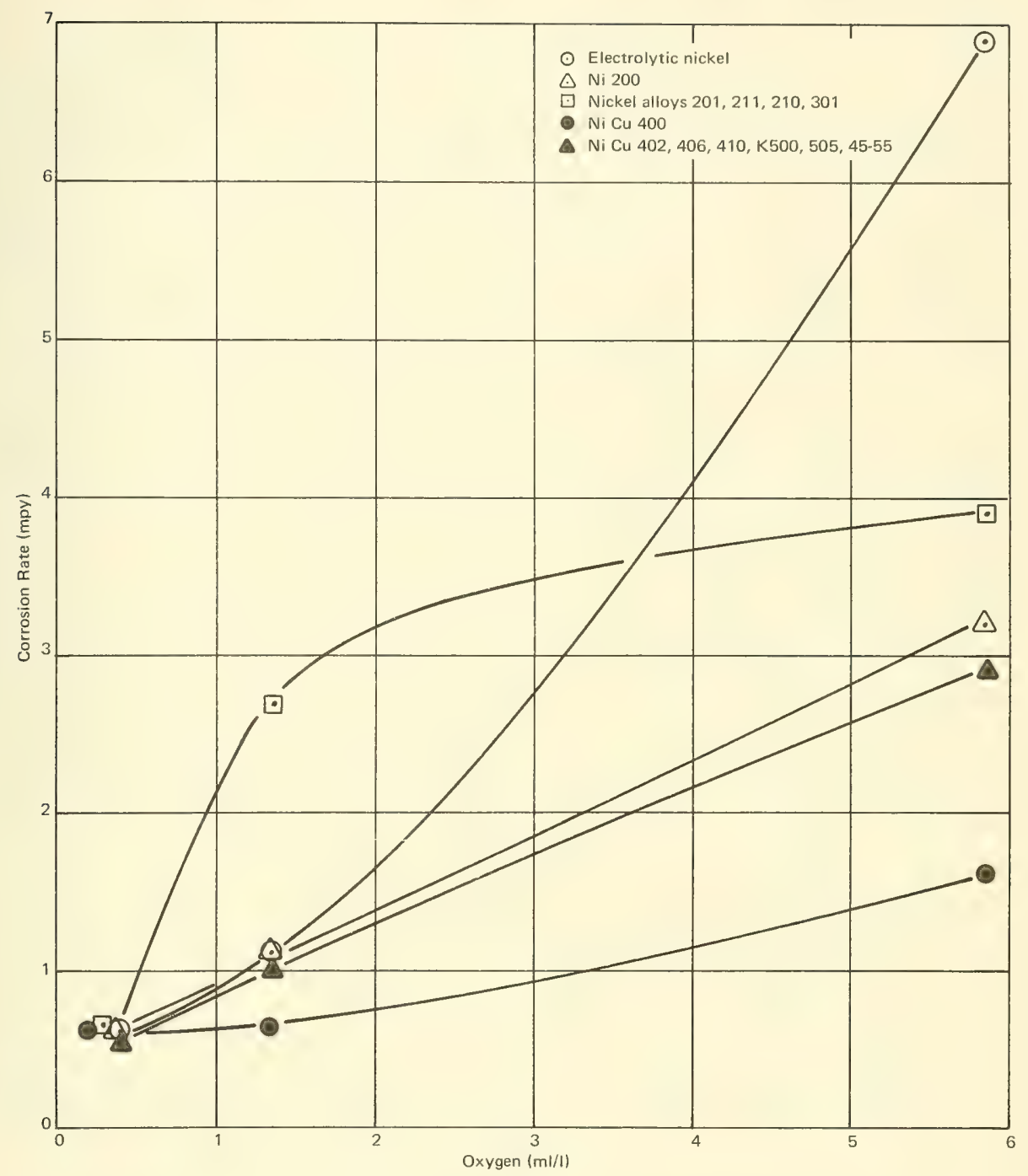

Figure 16. Corrosion of nickels and nickel-copper alloys vs oxygen content of seawater after 1 year of exposure. 


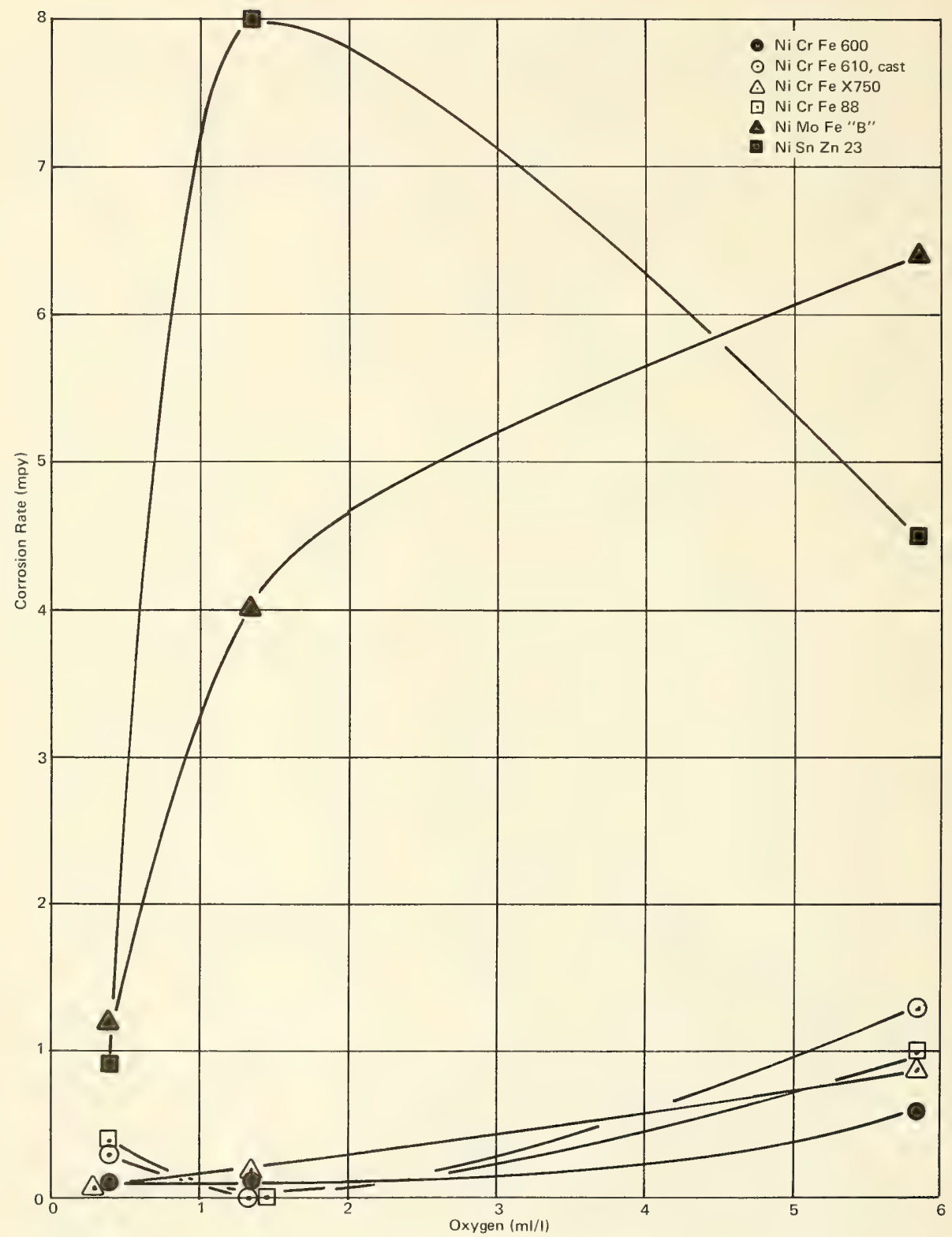

Figure 17. Corrosion of nickel alloys vs oxygen content of seawater after 1 year of exposure. 


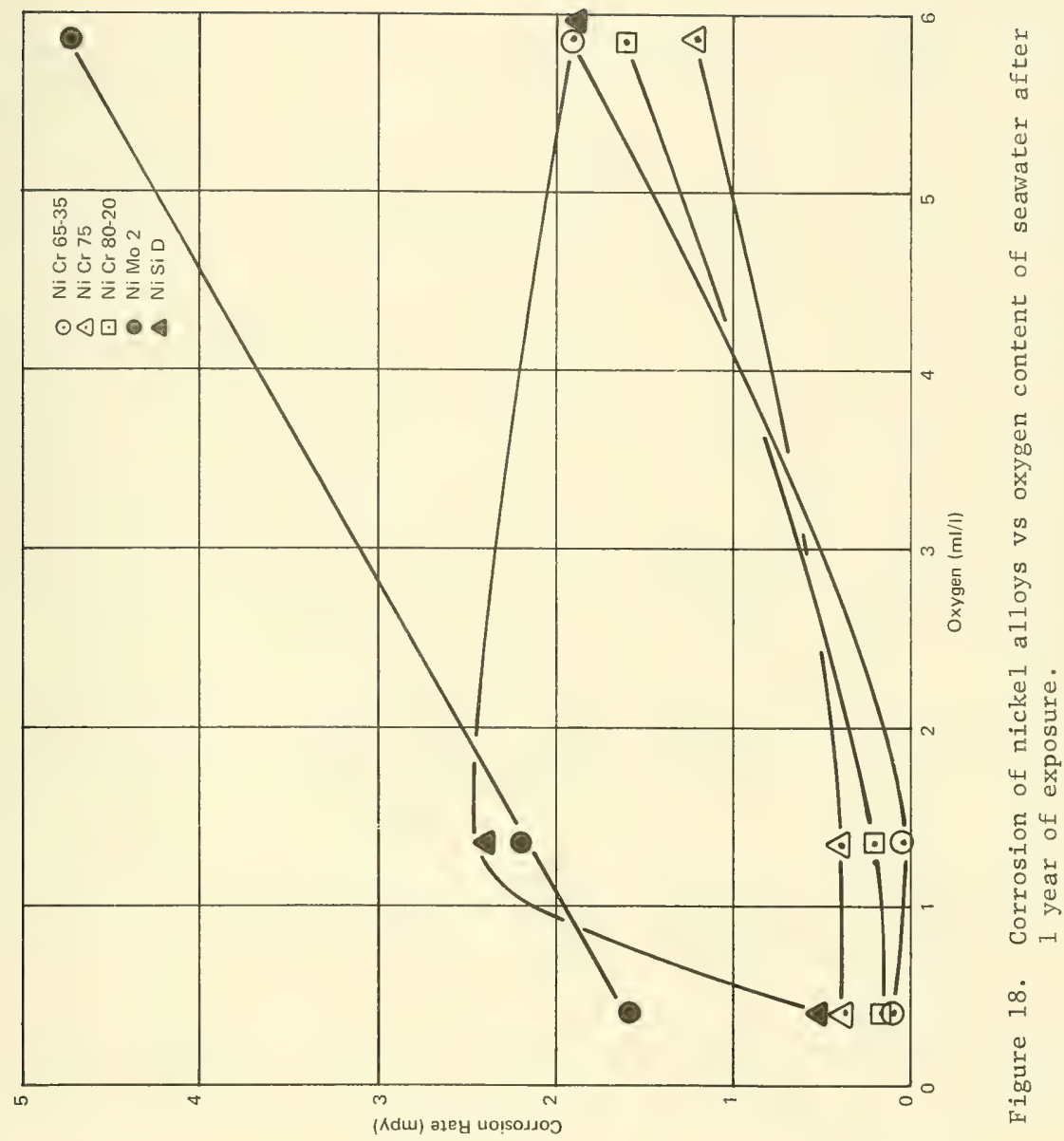




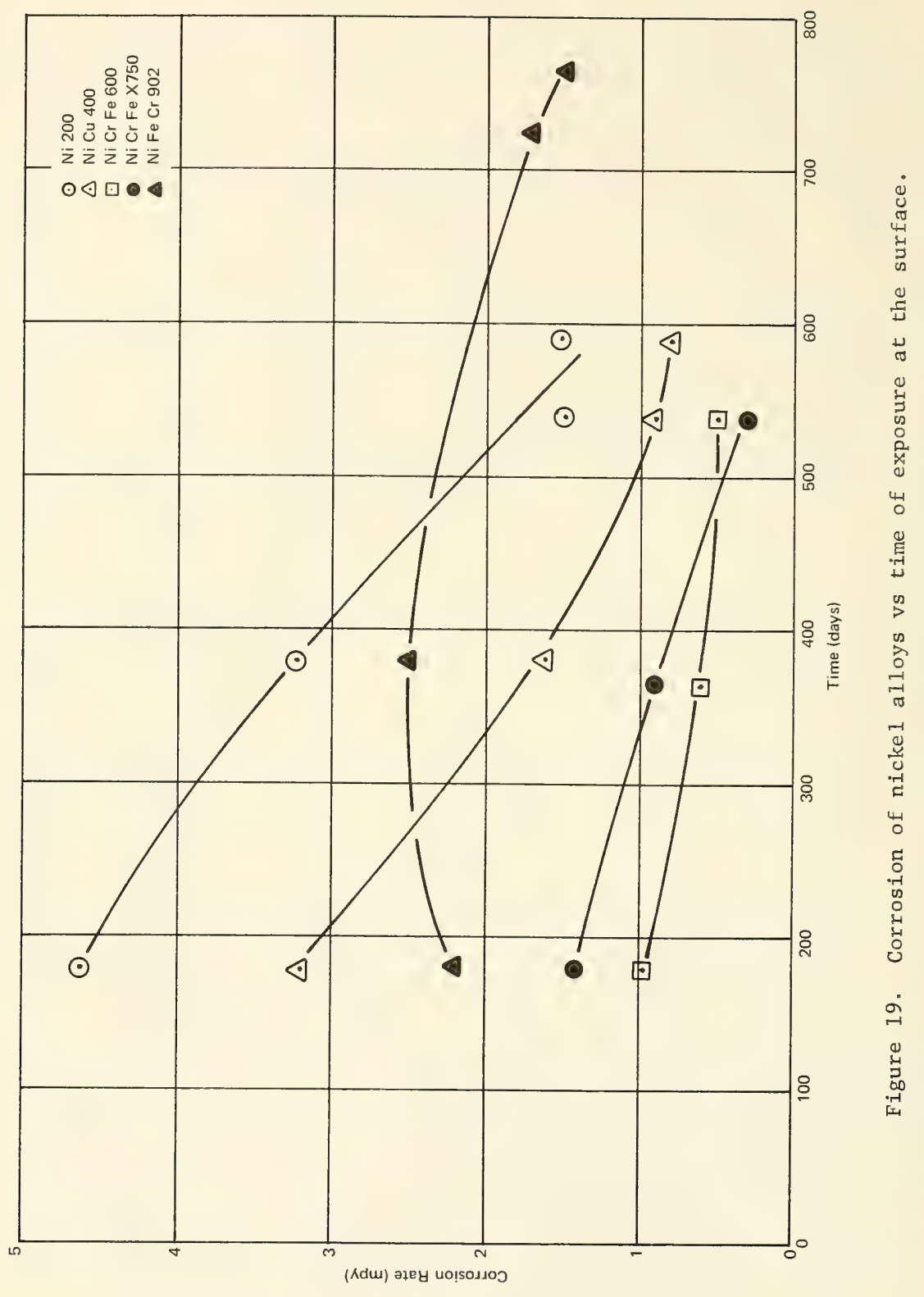




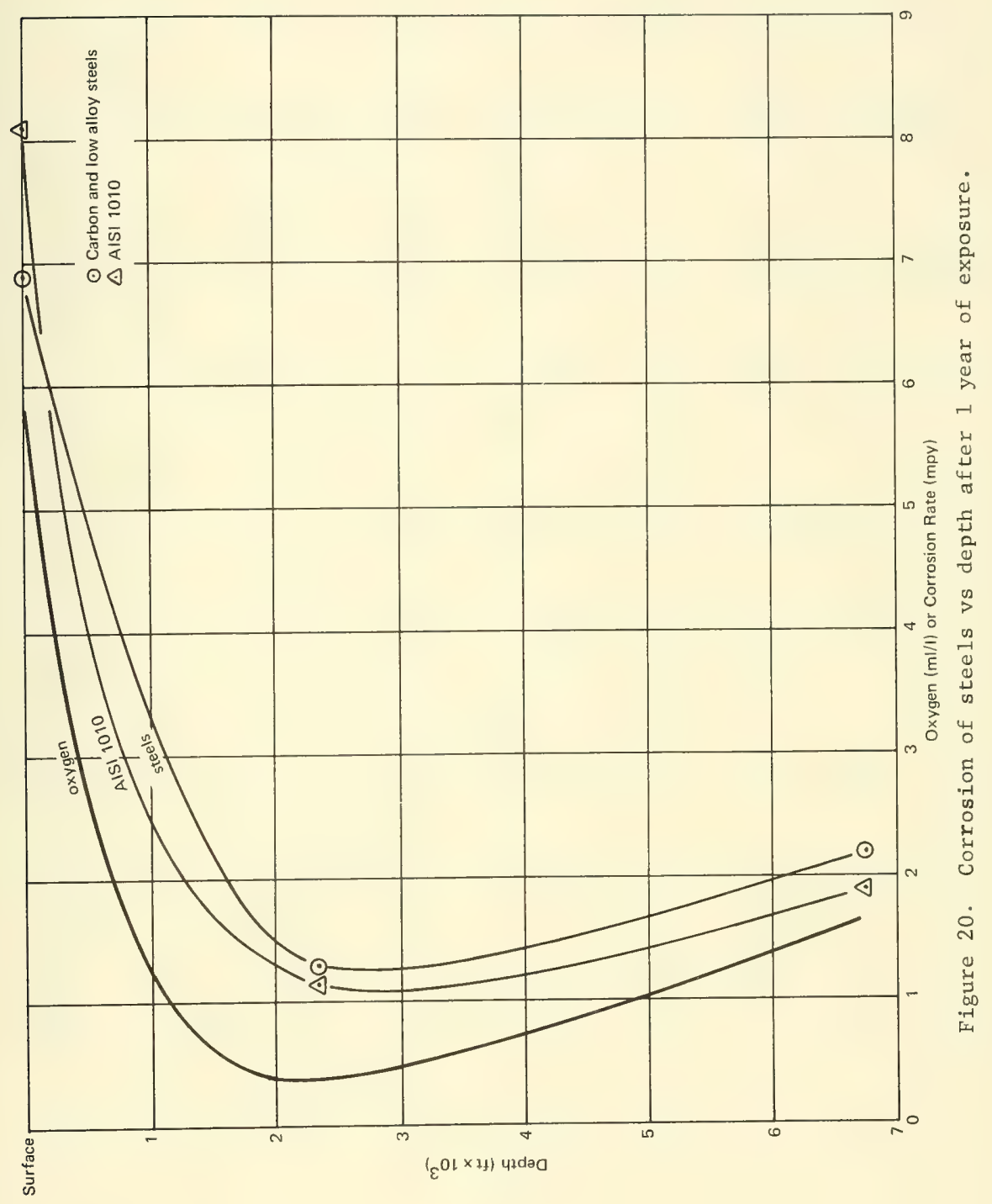




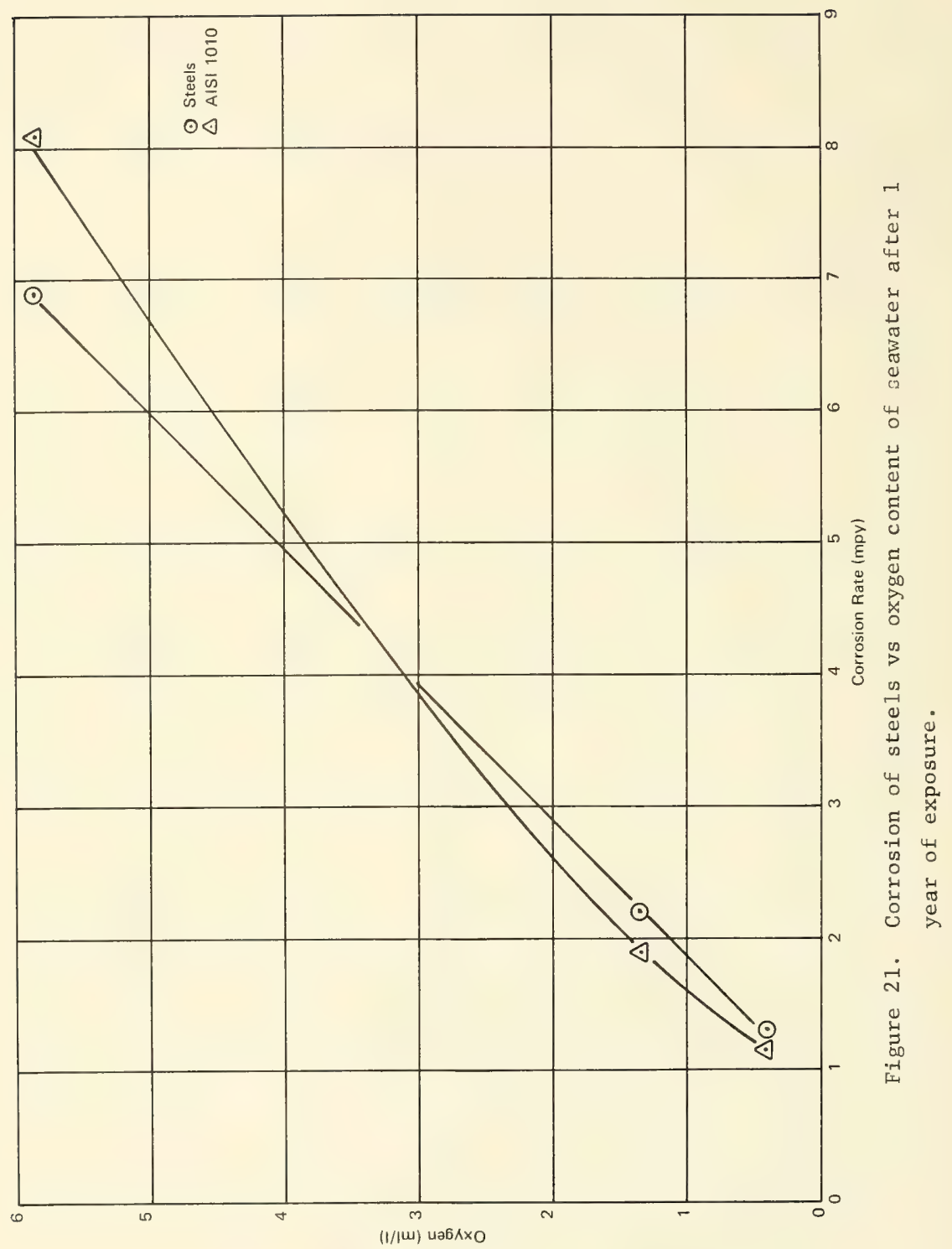




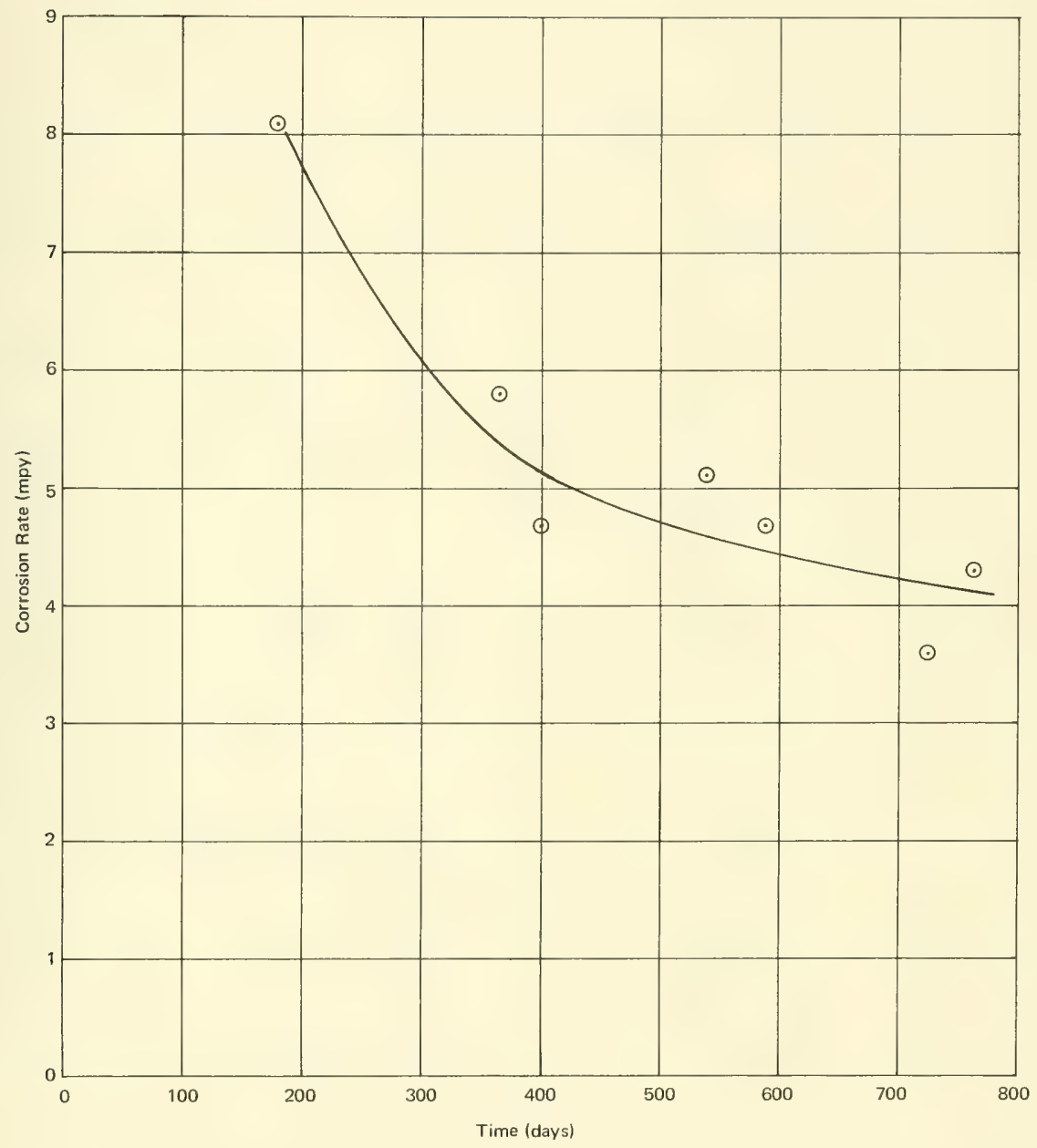

Figure 22. Corrosion of steels vs time of exposure at the surface. 


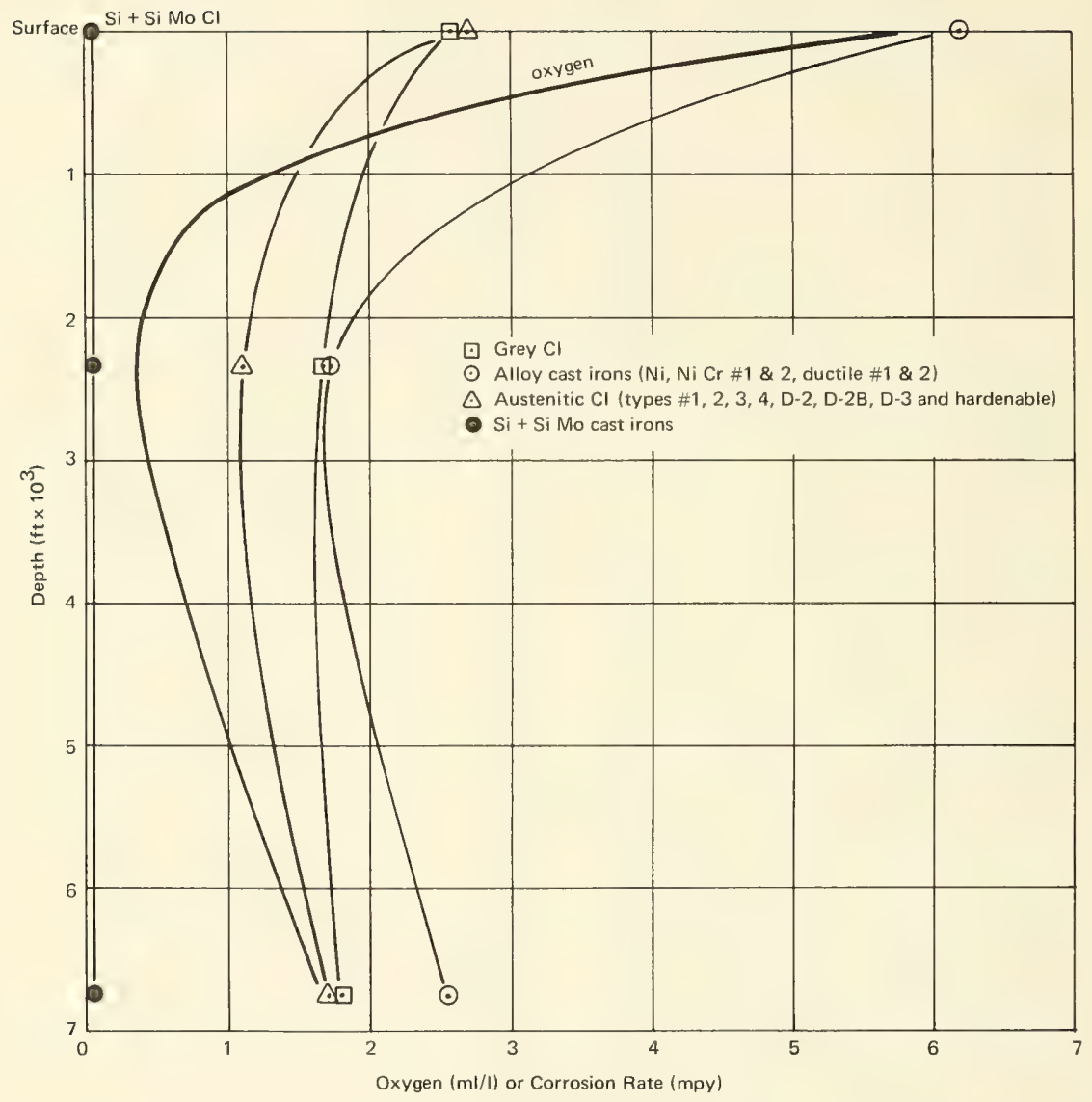

Figure 23. Corrosion of cast irons vs depth after 1 year of exposure. 


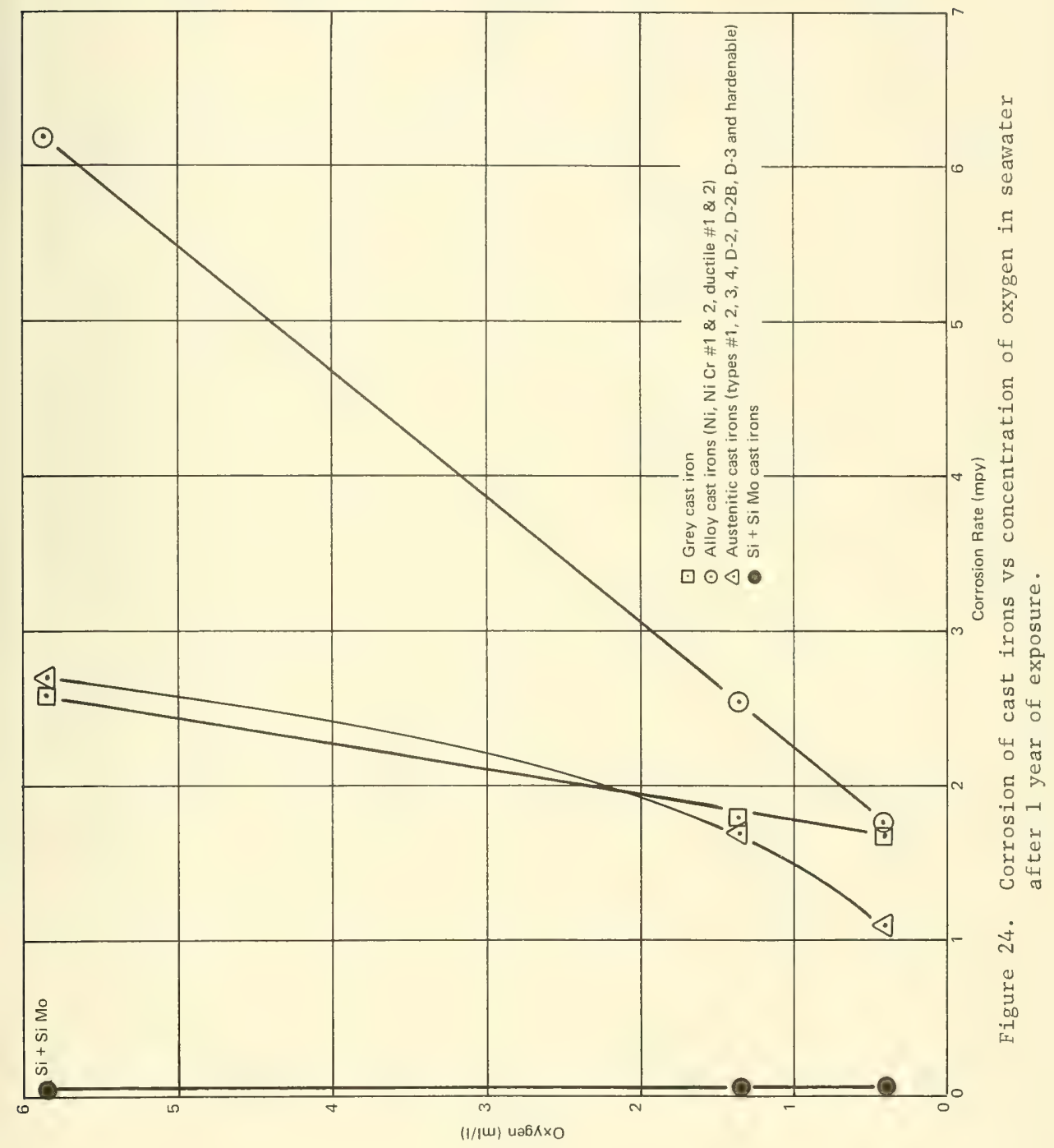




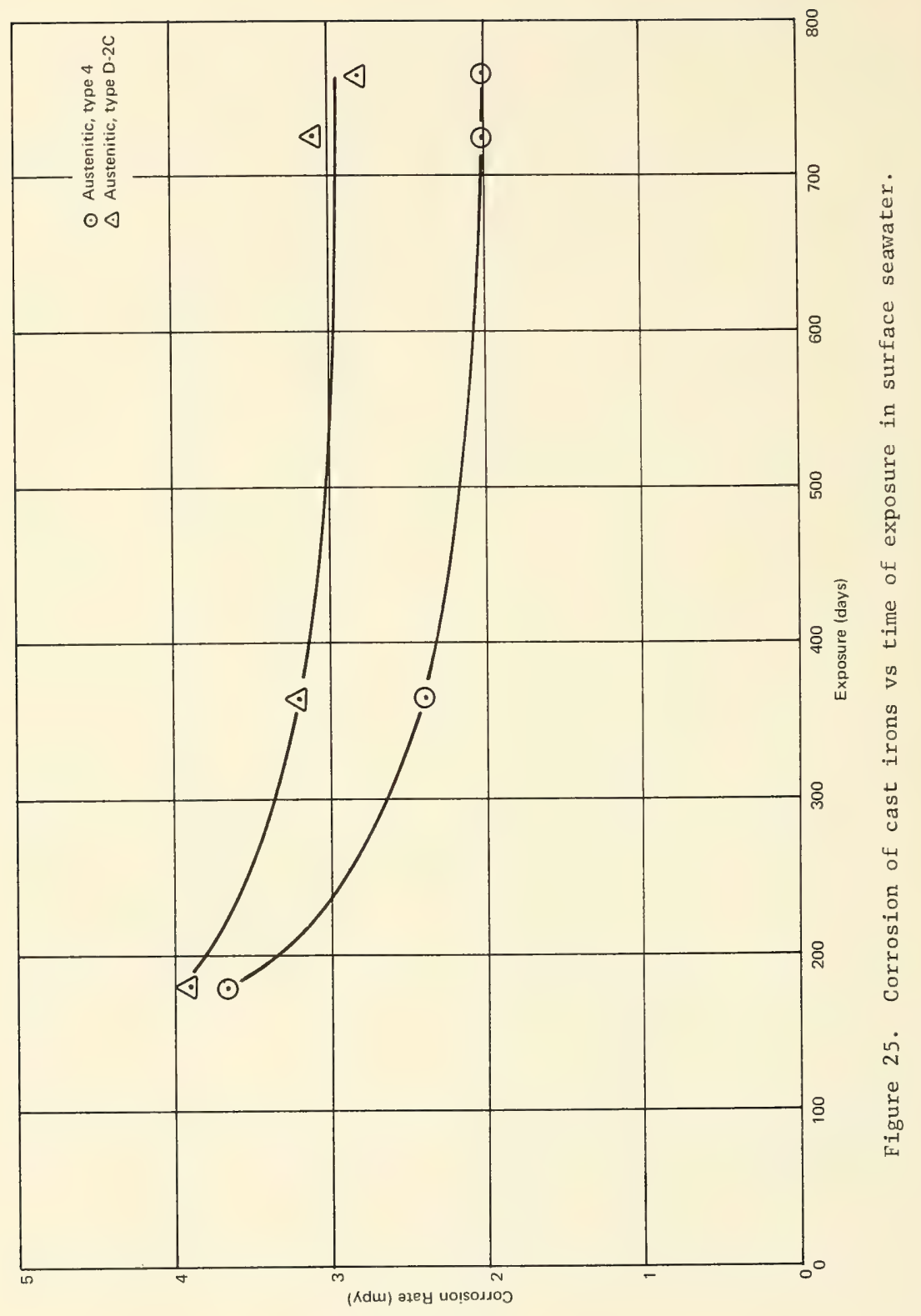




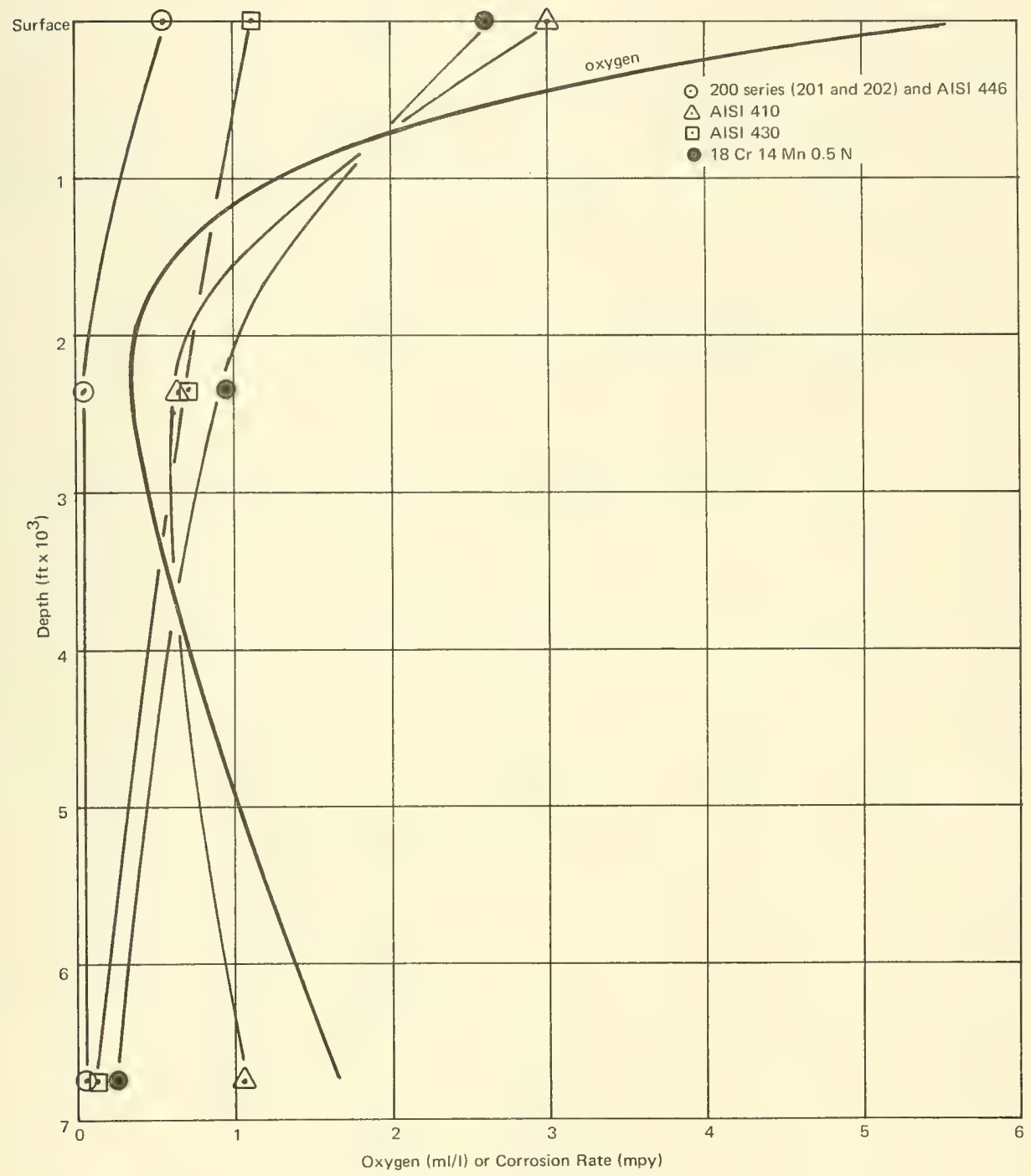

Figure 26. Corrosion of 200 and 400 Series stainless steels vs depth after 1 year of exposure. 


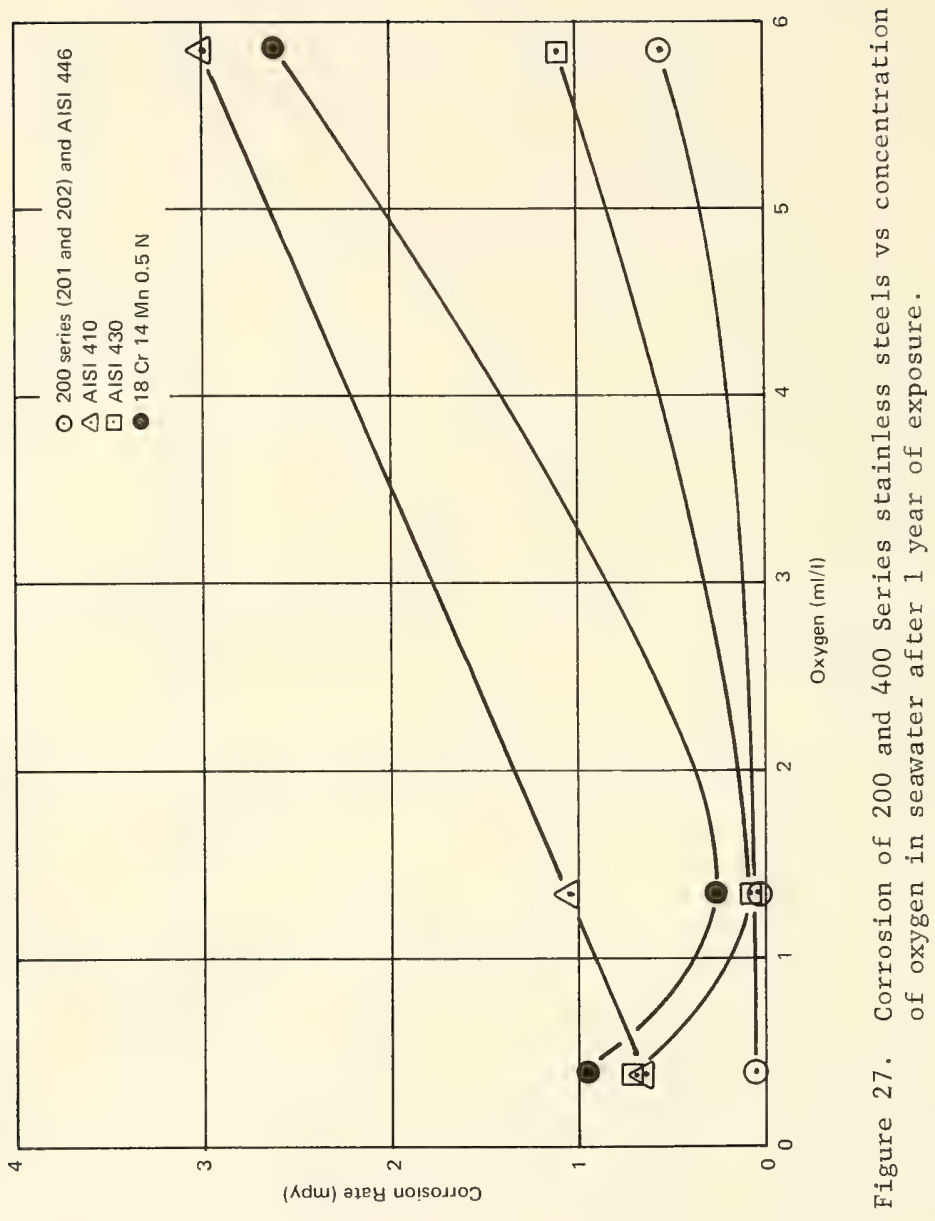




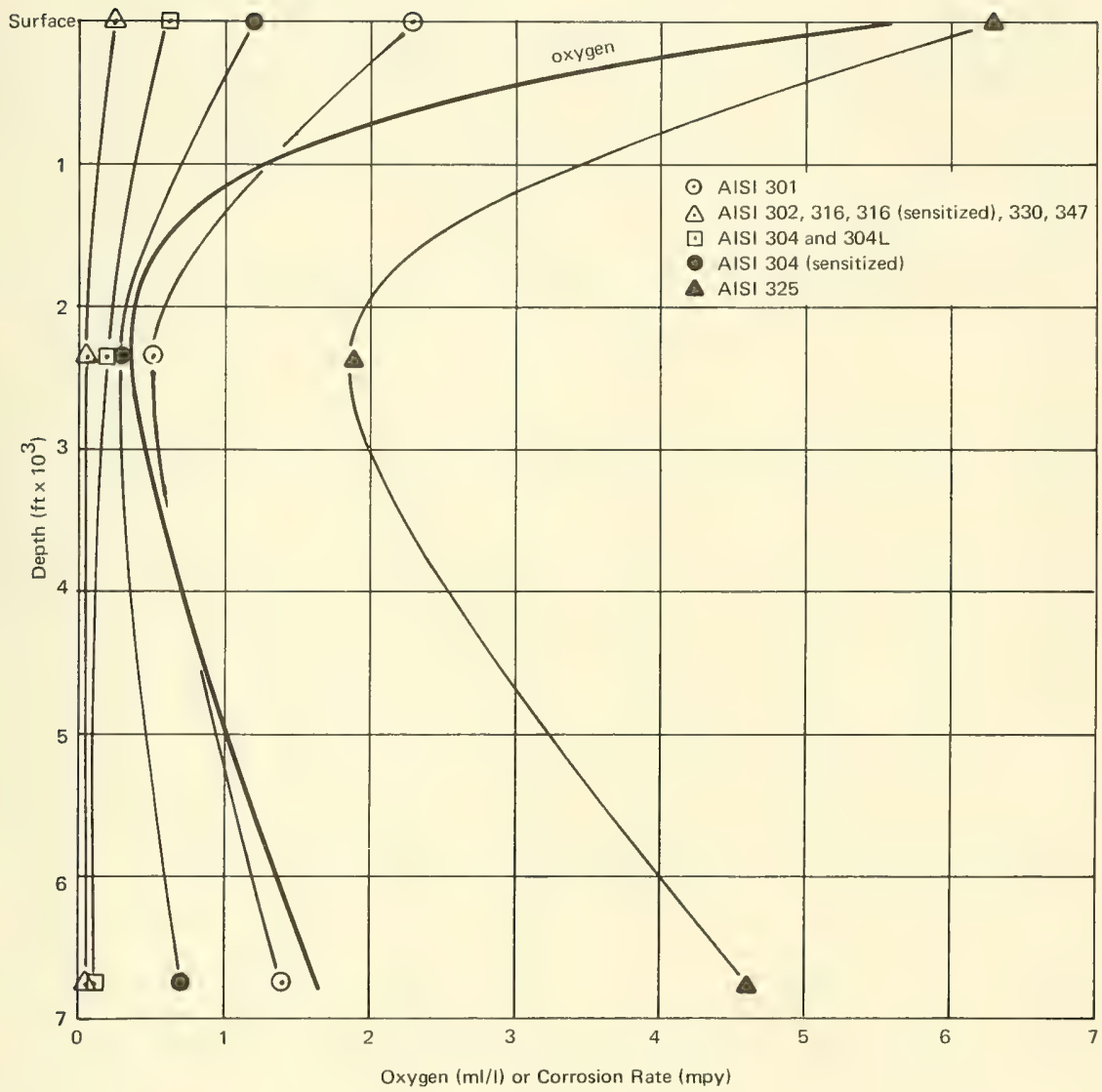

Figure 28. Corrosion of 300 Series stainless steels vs depth after 1 year of exposure. 


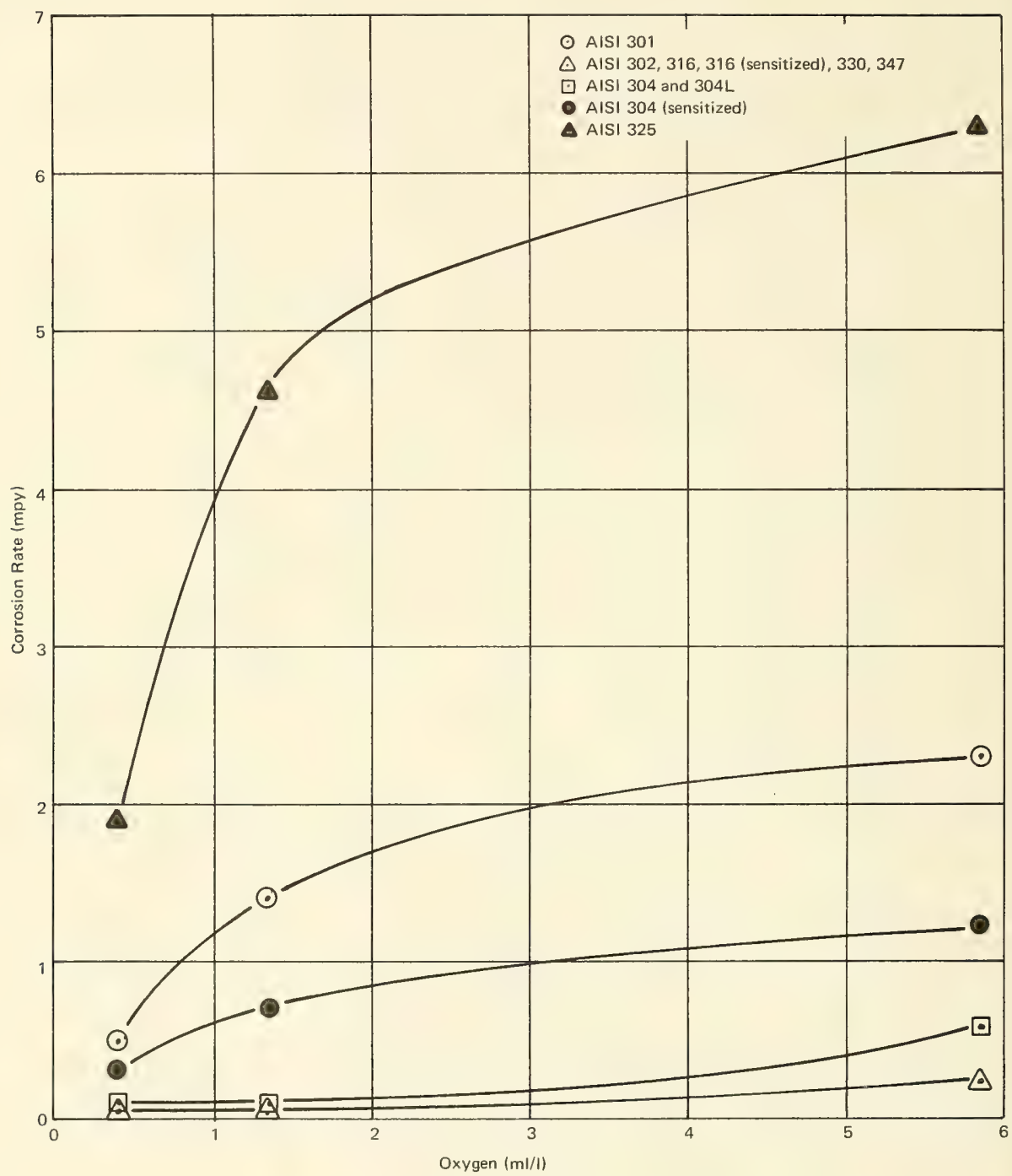

Figure 29. Corrosion of 300 Series stainless steels vs concentration of oxygen in seawater after 1 year of exposure. 


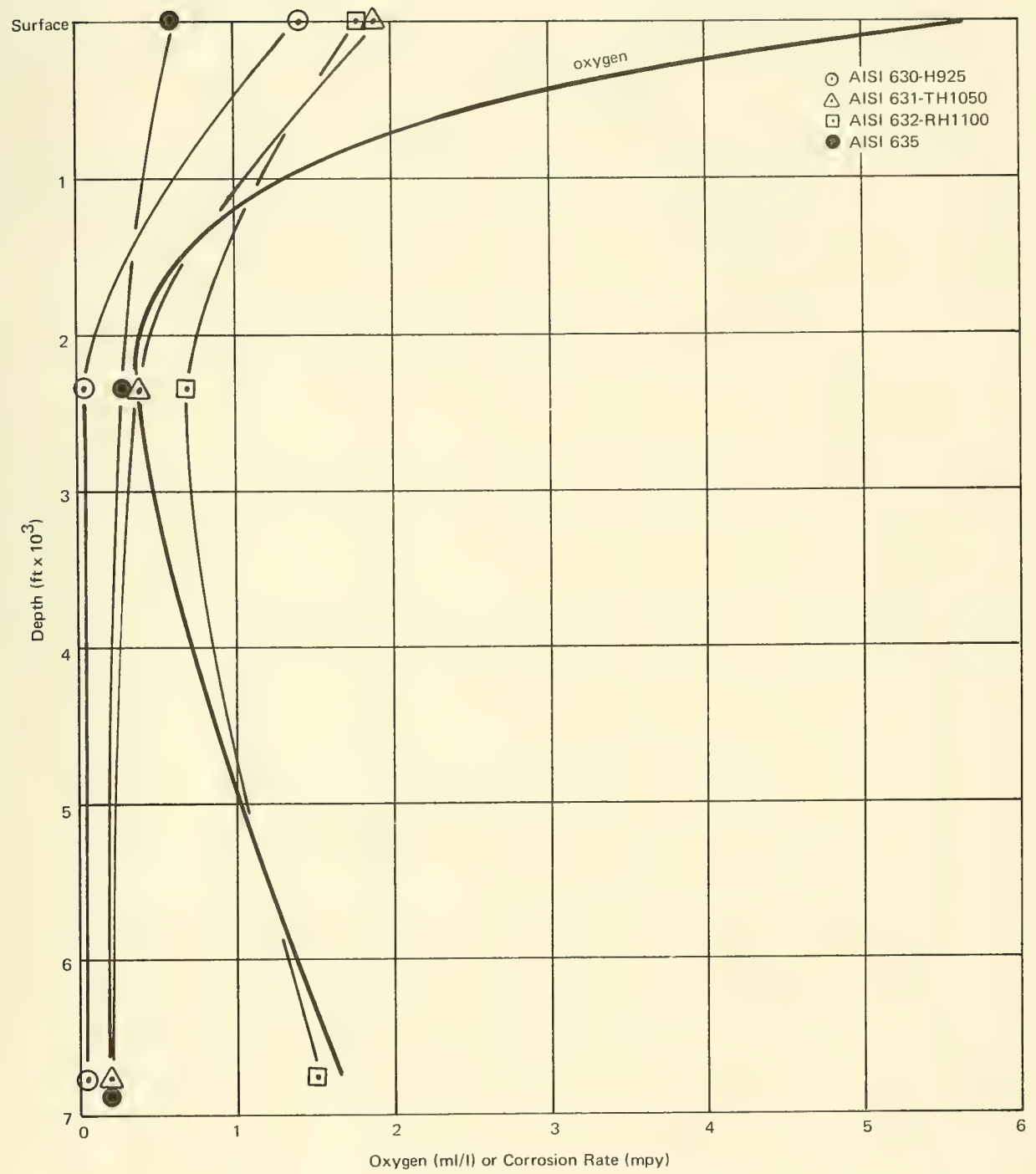

Figure 30. Corrosion of 600 Series stainless steels vs depth after 1 year of exposure. 


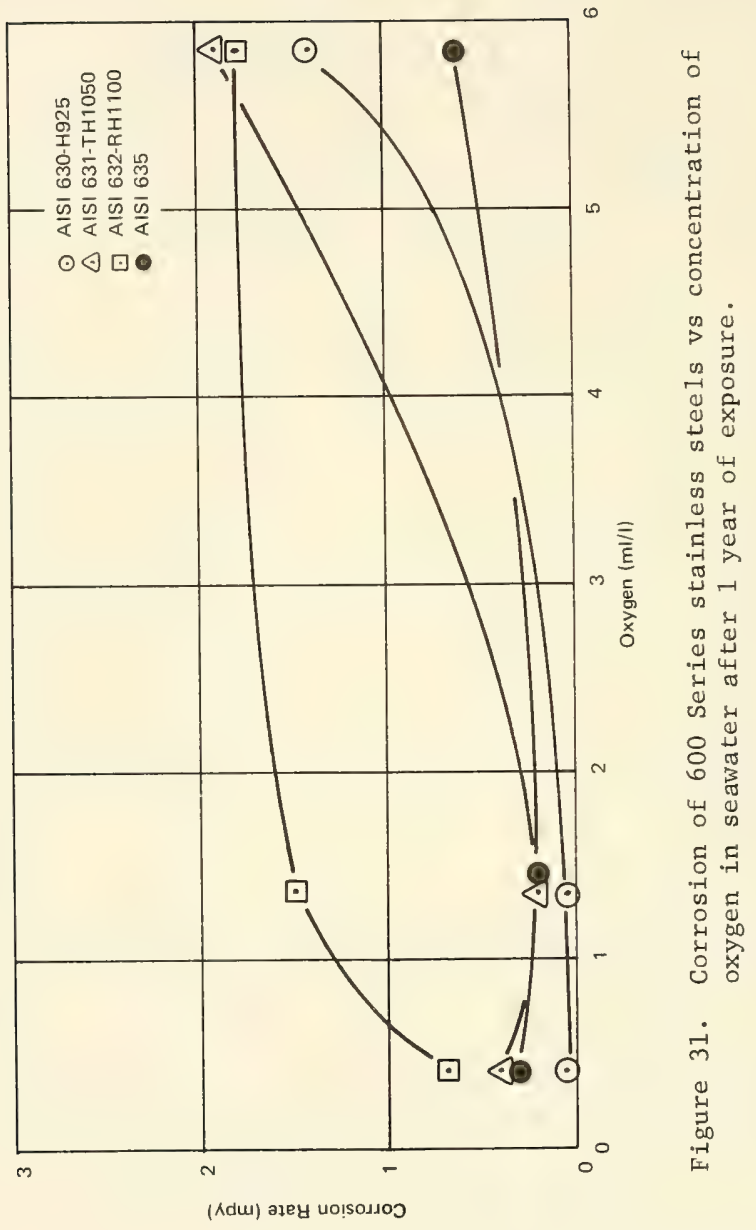




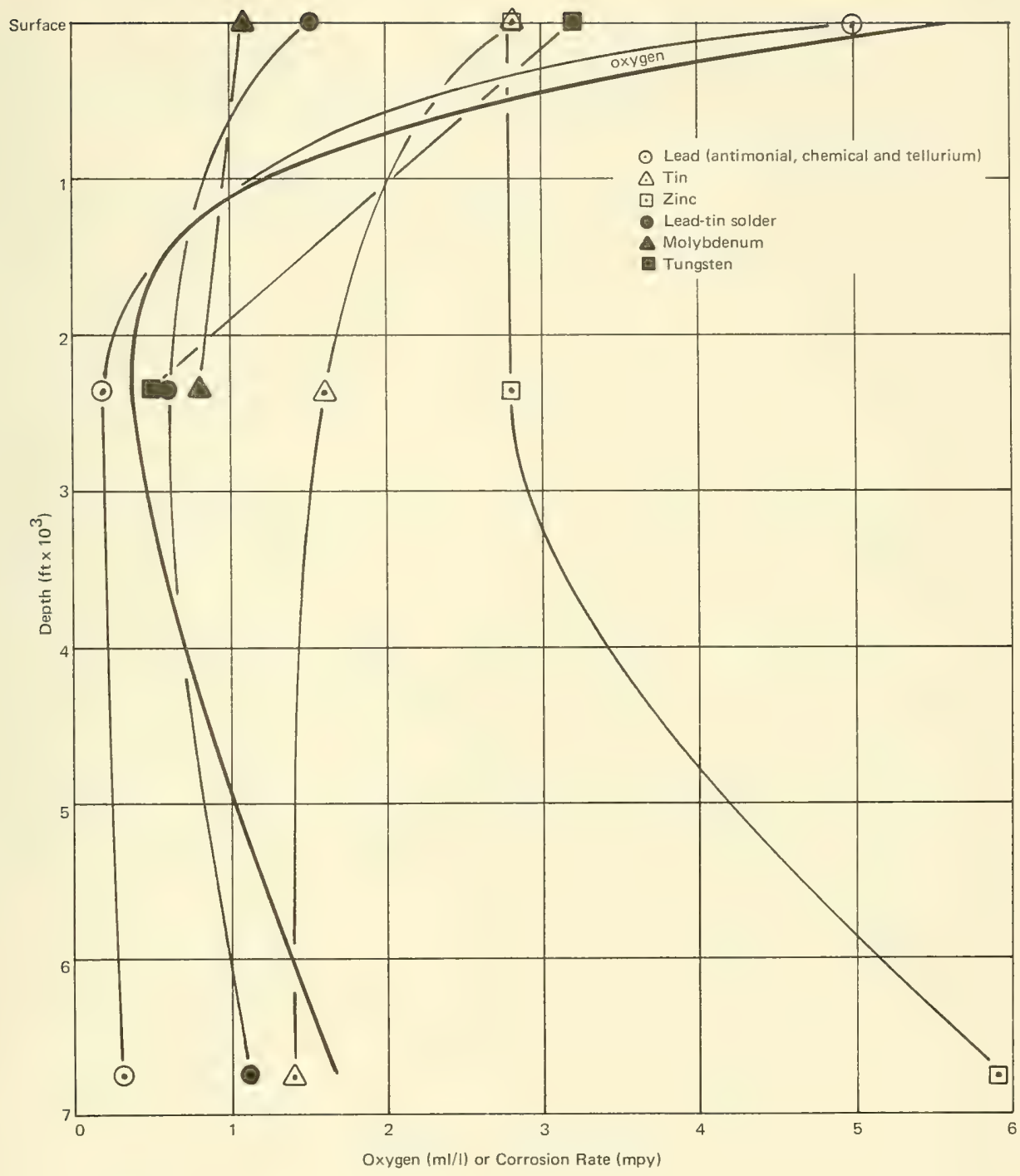

Figure 32. Corrosion of miscellaneous alloys vs depth after 1 year of exposure. 


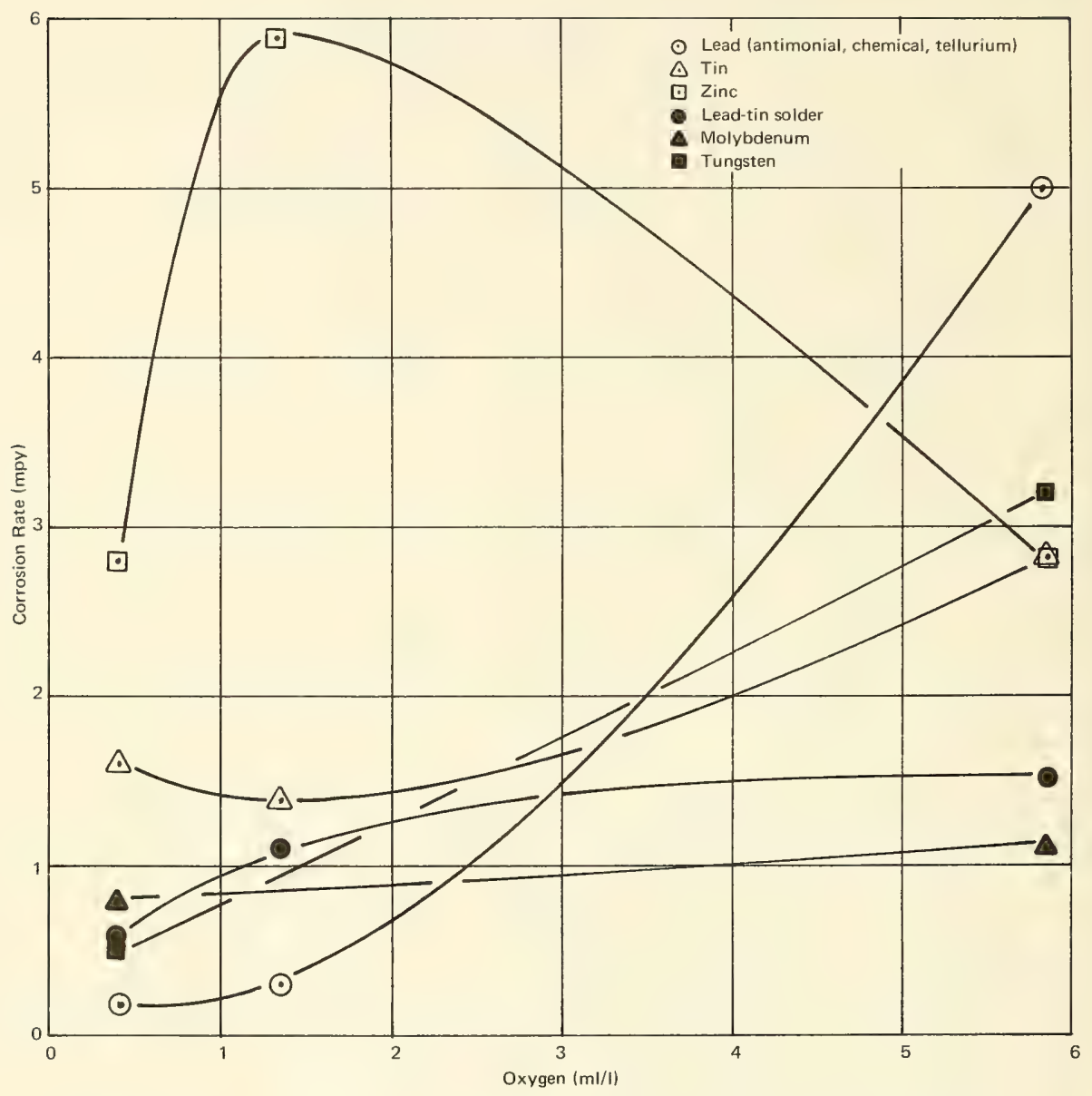

Figure 33. Corrosion of miscellaneous alloys vs concentration of oxygen in seawater after 1 year of exposure. 


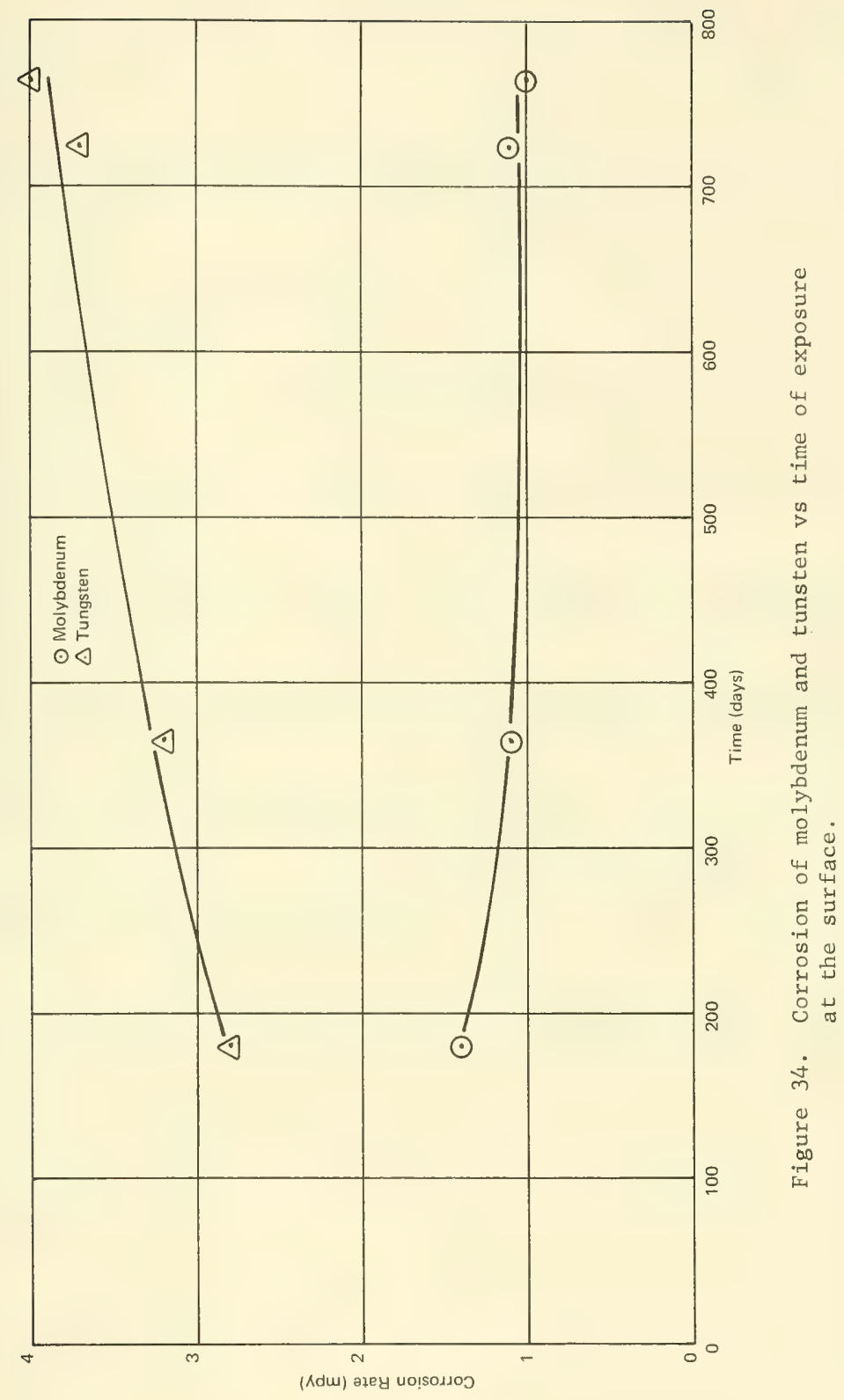




\section{REFERENCES}

1. Naval Civil Engineering Laboratory. Technical Note N-781: Effect of deep ocean environment on the corrosion of selected alloys, by Fred M. Reinhart. Port Hueneme, Ca., Oct 1965.

2. hydrospace, by Fred M. Reinhart. Port Hueneme, Ca., Dec 1966.

3. Technical Note N-900: Corrosion of materials in hydrospace - Part I - Irons, steels, cast irons and steel products, by Fred M. Reinhart. Port Hueneme, Ca., Jul 1967.

4. Technical Note N-915: Corrosion of materials in hydrospace - Part II - Nickel and nickel alloys, by Fred M. Reinhart. Port Hueneme, Ca., Aug 1967.

5. Technical Note N-921: Corrosion of materials in hydrospace - Part III - Titanium and titanium alloys, by Fred M. Reinhart. Port Hueneme, Ca. Sep 1967.

6. - Technical Note N-961: Corrosion of materials in hydrospace - Part IV - Copper and copper alloys, by Fred M. Reinhart. Port Hueneme, Ca., Apr 1968.

7. Technical Note N-1008: Corrosion of Materials in hydrospace - Part V - Aluminum alloys, by Fred M. Reinhart. Port Hueneme, Ca., Jan 1969.

8. Technical Note N-1023: Corrosion of materials in surface seawater after 6 months of exposure, by Fred M. Reinhart. Port Hueneme, Ca., Mar 1969.

9. Technical Note N-1172: Corrosion of materials in hydrospace - Part VI - Stainless steels, by Fred M. Reinhart. Port Hueneme, Ca., Jul 1971.

10. Dr. T. P. May. Unpublished data, International Nickel Co., Inc., New York City, N. Y. 


\section{DISTRIBUTION LIST}

\begin{tabular}{|c|c|c|c|}
\hline $\begin{array}{l}\text { SNDL } \\
\text { Code }\end{array}$ & $\begin{array}{c}\text { No. of } \\
\text { Activities }\end{array}$ & $\begin{array}{l}\text { Total } \\
\text { Copies }\end{array}$ & \\
\hline- & 1 & 12 & Defense Documentation Center \\
\hline FKAIC & 1 & 10 & Navai Facilities Engineering Command \\
\hline FKN1 & 6 & 6 & NAVFAC Engineering Field Divisions \\
\hline FKN5 & 9 & 9 & Public Works Centers \\
\hline FA25 & 1 & 1 & Public Works Centers \\
\hline- & 9 & 9 & $\begin{array}{l}\text { RDT\&E Liaison Officers at NAVFAC } \\
\text { Engineering Field Divisions and } \\
\text { Construction Battalion Centers }\end{array}$ \\
\hline- & 190 & 190 & $\begin{array}{l}\text { NCEL Special Distribution List } \\
\text { for persons and activities interested } \\
\text { in reports on Deep Ocean Corrosion }\end{array}$ \\
\hline
\end{tabular}




\section{DOCUMENT CONTROL DATA - R \& D}

(Securily classification of tille, body of abstract and indexind unnotalian nuust be entered when the overall report is clossified) 1. ORIGINATING ACTIVITY (CORPORate author)

Naval Civil Engineering Laboratory

Port Hueneme, California 93043 a. REFORT SECURITY CLASSIFICATION Unclassified 3. REPORT TITLE

CORROSION OF MATERIALS IN SURFACE SEAWATER AFTER 12 AND 18 MONTHS OF EXPOSURE

4. DESCRIP TIVE NOTES (TYPe of report and inclusive dates)

Final - June 1966-June 1971

5. AUTHOR(S) (First name, middlo initial, last name)

Fred M. Reinhart and James F. Jenkins

\begin{tabular}{|c|c|c|}
\hline $\begin{array}{l}\text { C. REPORT DATE } \\
\text { January } 1972\end{array}$ & $\begin{array}{l}\text { 78. TOTAL NO. OF PAGES } \\
103\end{array}$ & $\begin{array}{c}\text { 76. NO. OF REFS } \\
10\end{array}$ \\
\hline Ba. CONTAACT OR GRANT HO. & \multicolumn{2}{|c|}{ 98. ORIGINATOR'S REPORT NUMBERISI } \\
\hline b. PROJECT NO. YF 38.535 .005 .01 .004 & \multicolumn{2}{|c|}{ Technical Note N-1213 } \\
\hline $\begin{array}{l}\text { c. } \\
\text { d. }\end{array}$ & \multicolumn{2}{|c|}{$\begin{array}{l}\text { 96. OTHER REPORT NO(S) (Any other numbers that may bo as signed } \\
\text { this report) }\end{array}$} \\
\hline
\end{tabular}

Approved for public release; distribution unlimited

Naval Facilities Engineering Command

A total of 1150 specimens of 189 different alloys were completely immersed in surface seawater for 12 and 18 months to obtain data for comparison with deep ocean corrosion data.

Corrosion rates, types of corrosion and pit depths were determined.

Some highly alloyed nickel alloys, titanium alloys, silicon cast irons, specialty stainless steels, columbium, tantalum and a tantalumtungsten alloy were uncorroded in seawater both at the surface and at depth.

The corrosion rates of the copper base alloys, nickel base alloys, steels, cast irons, lead, tin, lead-tin solder, molybdenum and tungsten decreased with the concentration of oxygen in seawater, i.e., the corrosion rates were lower at depth than at the surface. The corrosion rates of $\mathrm{Ni}-200, \mathrm{Ni}-\mathrm{Cu} 402,410, \mathrm{~K}-500$ and 45-55, $\mathrm{Ni}-\mathrm{Cr}-\mathrm{Fe}$ $\mathrm{X} 750, \mathrm{Ni}-\mathrm{Mo} 2$, a11 steels, grey cast iron and alloy cast irons decreased linearly with the concentration of oxygen in seawater.

The copper base alloys, steels, cast irons, molybdenum, tungsten, lead and lead-tin solder corroded uniformly both at the surface and at depth.

Continued

\section{DD FORM 1473 (PAGE 1)}

$S / N 0101.807 .6801$
Unclassified 
Unclassified

Security Classification

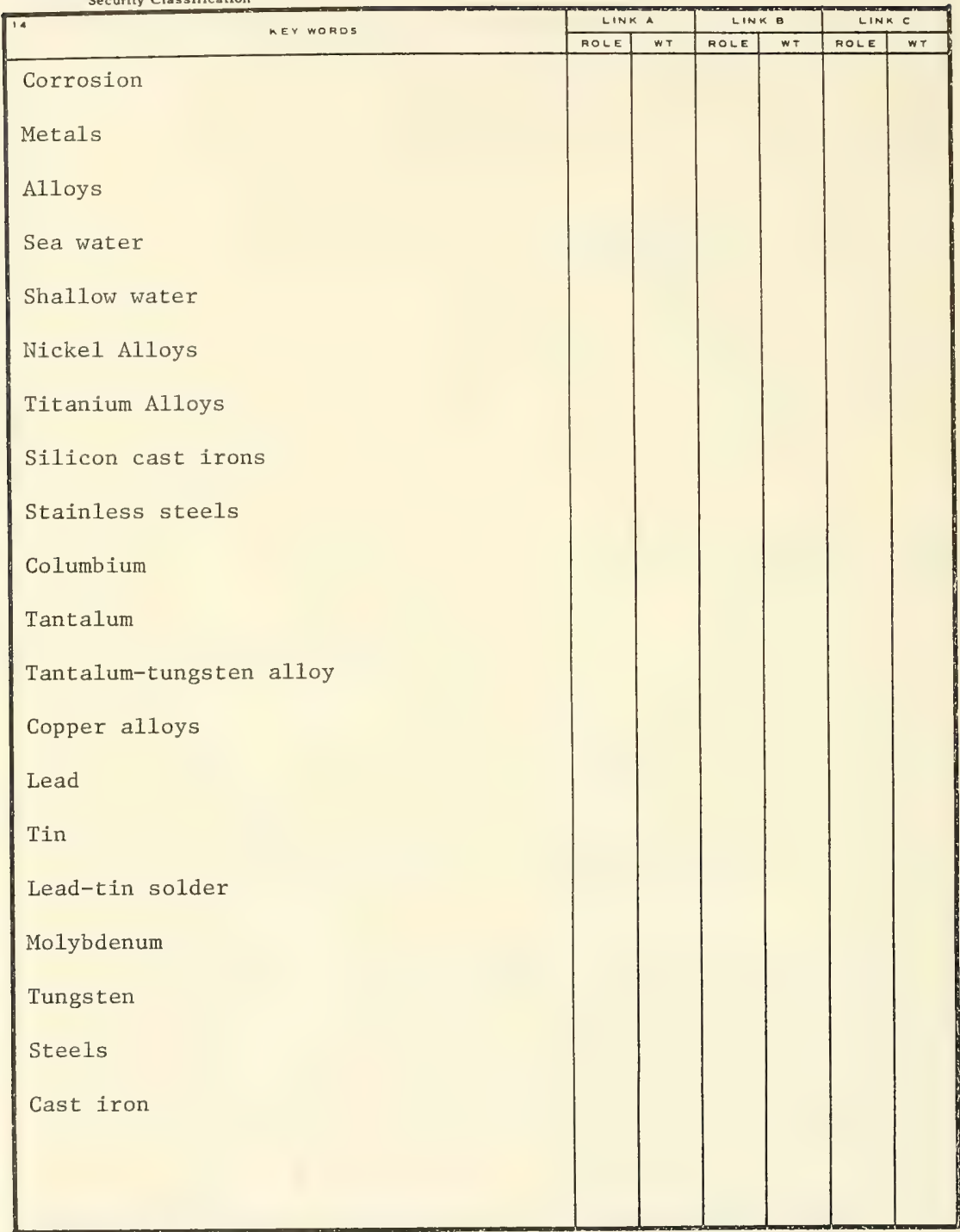

\section{DD, FORM 9 IA73 (BACK)}

Unclassified

(PAGE 2) 
The aluminum alloys were attacked by pitting and crevice corrosion and seawater was more aggressive at depth than at the surface for such alloys. The effect of the concentration of oxygen in seawater on the corrosion of aluminum alloys was inconsistent.

The stainless steels were attacked by pitting, tunneling and crevice corrosion, except 309, 316L, 317, 329, 633, 0 Cb-3 and Ni-Cr-Mo-Si.

Surface seawater was more aggressive than seawater at depth in promoting these types of corrosion on the stainless steels. 

Technical Note $\mathrm{N}-1213$

Naval Civil Engineering Laboratarur Corrosion of Materials in Surface Seawater after 12 and 18 Henths of Exposure 
